\title{
Elementary Visual Hallucinations and Their Relationships to Neural Pattern-Forming Mechanisms
}

\author{
Vincent A. Billock and Brian H. Tsou \\ U.S. Air Force Research Laboratory
}

\begin{abstract}
An extraordinary variety of experimental (e.g., flicker, magnetic fields) and clinical (epilepsy, migraine) conditions give rise to a surprisingly common set of elementary hallucinations, including spots, geometric patterns, and jagged lines, some of which also have color, depth, motion, and texture. Many of these simple hallucinations fall into a small number of perceptual geometries-the Klüver forms - that (via a nonlinear mapping from retina to cortex) correspond to even simpler sets of oriented stripes of cortical activity (and their superpositions). Other simple hallucinations (phosphenes and fortification auras) are linked to the Klüver forms and to pattern-forming cortical mechanisms by their spatial and temporal scales. The Klüver cortical activity patterns are examples of self-organized pattern formation that arise from nonlinear dynamic interactions between excitatory and inhibitory cortical neurons; with reasonable modifications, this model accounts for a wide range of hallucinated patterns. The Klüver cortical activity patterns are a subset of autonomous spatiotemporal cortical patterns, some of which have been studied with functional imaging techniques. Understanding the interaction of these intrinsic patterns with stimulus-driven cortical activity is an important problem in neuroscience. In line with this, hallucinatory pattern formation interacts with physical stimuli, and many conditions that induce hallucinations show interesting interactions with one another. Both types of interactions are predictable from neural and psychophysical principles such as localized processing, excitatory-inhibitory neural circuits, lateral inhibition, simultaneous and sequential contrast, saccadic suppression, and perceptual opponency. Elementary hallucinations arise from familiar mechanisms stimulated in unusual ways.
\end{abstract}

Keywords: intrinsic neural activity, Klüver form constant, migraine fortification aura, phosphene, spatiotemporal pattern formation

What are these Geometrical Spectra? and how, and in what department of the bodily or mental economy do they originate? ... there is a kaleidoscopic power in the sensorium to form regular patterns by the symmetrical combination of causal elements....

—The astronomer Sir John F. W. Herschel (1867) on hallucinations seen during migraine and during surgery "under the blessed influence of chloroform."

I had two days spoiled by a psychological experiment with mescal, an intoxicant used by some of our Southwestern Indians in their religious ceremonies; a sort of cactus bud, of which the U.S. Government had

This article was published Online First March 26, 2012.

Vincent A. Billock, National Research Council, U.S. Air Force Research Laboratory, Wright-Patterson Air Force Base, Ohio; Brian H. Tsou, U.S. Air Force Research Laboratory, Wright-Patterson Air Force Base, Ohio.

This research was funded in part by a National Research Council/Air Force Office of Scientific Research Senior Research Award to Vincent A. Billock. We thank Scott Kelso and Mingzhou Ding for introducing us to this topic; Oliver Sacks for suggesting that we look at relationships between patterns formed under migraine and image stabilization; Angela Brown, Lynn Olzak, Keith White, and Ewen King-Smith for helpful discussions on migraine, functional imaging limitations, and retinal sampling; and Bard Ermentrout and Whitman Richards for comments on an earlier version of the manuscript.

Correspondence concerning this article should be addressed to Vincent A. Billock, NRC, Room 210, Building 248, Wright-Patterson Air Force Base, OH 45433. E-mail: vincent.billock.ctr@wpafb.af.mil distributed a supply to certain medical men, including Weir Mitchell, who sent me some to try. He himself had been in "fairyland." It gives the most glorious visions of color. ... I took one bud three days ago, was violently sick for 24 hours, and had no other symptoms. . . . I will take the visions on trust!

-William James (1920) in a June 11, 1896, letter to his brother

Henry.

For many, mention of visual hallucinations brings to mind complex visual imagery: the stuff of waking dreams. This is misleading; simple forms and patterns are more typical than Freudian drama. Elementary hallucinations-generated by a host of clinical and laboratory-induced states-range from simple spots to the flashing serrated arcs that accompany migraines to the kaleidoscopic patterns induced by some drugs. Until recently, this sheer variety made hallucinations seem like a collection of disparate curiosities ill-posed for serious study. However, there are at least four reasons why this view is mistaken. First, there is much more order in these conditions than is generally appreciated. There are many links and interactions between hallucinatory conditions. Moreover, even very different-seeming elementary hallucinations take place on common spatial and temporal scales that correspond to identifiable neural mechanisms. Indeed, some different-seeming hallucinations can have the same pattern of cortical activity, differing only by cortical position and orientation. The strong connections between elementary visual hallucinations and particular neural systems led Frances Wilkinson (2004) to call hallucinations 
"windows on the visual brain." Second, elementary hallucinations illustrate an important general principle in cognitive science. It is now well recognized that complex systems, such as the human brain, have collective properties that are not inherent in the individual neural elements; it has become common to ascribe some cognitive behaviors as emergent properties of the collective system. However, such attributions are seldom well worked out and are most often reasoned by analogy to better studied complex physical systems. Elementary hallucinations-and especially geometric hallucinations-provide particularly well-worked-out examples of a neural emergent behavior (self-organized spatiotemporal pattern formation) that corresponds to specific percepts. Third, a rather perverse aspect of the study of the human brain as a complex system is that the brain typically behaves in a deceptively stable and veridical fashion-much like a linear system; if we want to understand the brain as a nonlinear dynamic complex system we need to push it outside of its normal pseudolinear regime. ${ }^{1}$ Perceptual catastrophe then becomes a tool of systems identification. Finally, recent advances in neuroscience have given new importance to understanding the relationship between stimulus-driven and autonomous neural pattern formation. A convergence of research in complexity theory, neural modeling, visual psychophysics, and functional brain imaging dramatically advances understanding of perceptual pattern formation and highlights interactions between intrinsic neural activity and physical stimulation.

\section{Hallucinatory Conditions and the Percepts They Induce}

\section{An Overview of Elementary Hallucinatory Visual Percepts}

This review focuses on elementary hallucinations and excludes complex imagery associated with dreams, dementia, delirium, and the latter phases of drug intoxication and sensory deprivation (useful reviews of complex hallucinations include Aleman \& Larøi, 2008; Collerton, Perry, \& McKeith, 2005; Horowitz, 1978). ${ }^{2}$ We also exclude the fascinating disturbances of shape, size, motion, and color that accompany fever and neurological disorders (e.g., the Alice in Wonderland syndrome that can herald mononucleosis). An amazing variety of external and internal conditions lead to elementary hallucinations (see Figure 1 and Table 1). A partial list includes migraine, epilepsy, hypoglycemia, the early stages of some drug intoxications, ocular pressure, retinal and cortical electric stimulation, transcranial magnetic stimulation (TMS), and photopic stimulation, especially by uniform flickering lights. The ubiquity, ease of generation, and geometric nature of these hallucinations have been a clue to neural modeling and a goad to perceptual experimentation. There are three basic types of elementary hallucination: phosphenes, geometric forms, and fortifications. The simplest illusory forms are phosphenes-usually small spots of light-often induced by focal stimulation of the eye or brain (e.g., by ocular pressure, electricity, and, more exotically, cosmic rays; Sannita, Narici, \& Picozza, 2006). Phosphenes vary in size with position in the visual field and have a variety of shapes, colors, and movements. The ability to generate phosphenes has been an opportunity to those who would create visual pros- theses for the blind and a nuisance for those who would keep the induced percepts simple. Often, phosphenes are induced in great numbers (polyopia) and are organized by some geometric principle (see, e.g., Figure 1E); they may appear to be hexagonally packed or to be texture elements on some greater geometric figure. The most common geometric figures-the Klüver forms-are lattices (often hexagonal), polar webs, fan shapes, pinwheels, spirals, concentric circles, and some three-dimensional analogues (tunnels, funnels, etc.). Fortifications are bright flashing serrated arc-shaped patterns often seen in migraine; occasionally, similar zigzag forms, like a piece of a fortification arc, are seen in epileptic seizure, hypoglycemia, and other conditions (Klüver, 1966; Purkinje, 1819/1823). When hallucinations accompany migraine or epilepsy, they are referred to clinically as auras.

\section{Migraine and Fortification Illusions}

Migraine hallucinations range from Klüver's geometric forms to complex multisensory hallucinations (which resemble temporallobe epilepsy). Even within an observer, more than one hallucination can occur; for example, Oliver Sacks and John Herschel reported both Klüver geometries and fortification patterns, sometimes during the same attack (Sacks, 1995a). In subjects experiencing more than one percept, phosphenes are generally seen before fortifications, which are seen before Klüver patterns (Sacks, 1995b); geometric patterns, like lattices, are also sometimes glimpsed between fortification features. However, as fortification auras are considered characteristic of migraine, we focus on fortifications here. Fortifications are bright line segments set at roughly $60^{\circ}$ angles to one another along a vertical arc (see Figure $1 \mathrm{H})$. In migraine, many patients report that the line segments flicker at about $10 \mathrm{~Hz}$, but most patients have no experience in accurately naming flicker rates. Crotogino, Feindel, and Wilkinson (2001) flicker-matched migraine scintillations to a physical standard in 11 observers: The average matched scintillation rate was $17.8 \mathrm{~Hz}$, and individual subjects made consistent flicker matches across attacks. The segments are usually bright white, but colors can sometimes be seen between them (Sacks, 1995a) or on their tips, like match-ends; red, yellow, and blue, in that order, are most common (Richards, 1971). The jagged structure resembles an old style of fortification with many angled sides to deprive primitive wall-breaching artillery of a clean nonglancing shot. The illusion generally starts near the fovea in one visual hemifield and moves slowly toward the periphery. Near the fovea, the angled features are small, and the arc is nearly circular. The angles between the serrations are between $45^{\circ}$ and $60^{\circ}$. As the arc moves toward the periphery, its individual angular features enlarge, and the arc straightens into a nearly vertical shape that appears to move more quickly through the visual field. Dahlem and Müller's (2003)

\footnotetext{
${ }^{1}$ The required push may not be very large. There is some evidence that the cortex is critically poised, in the sense that relatively small changes can trigger avalanches of neuronal activity (Plenz \& Thiagarajan, 2007; Werner, 2007). This is in accord with the relatively modest amount of stimulation needed to induce some hallucinations.

${ }^{2}$ Some investigators report that complex hallucinations can develop from simpler ones (e.g., a geometric pattern of radiating lines becomes the legs of a spider upon introspection; Horowitz, 1978; Hughlings Jackson, 1958).
} 
A
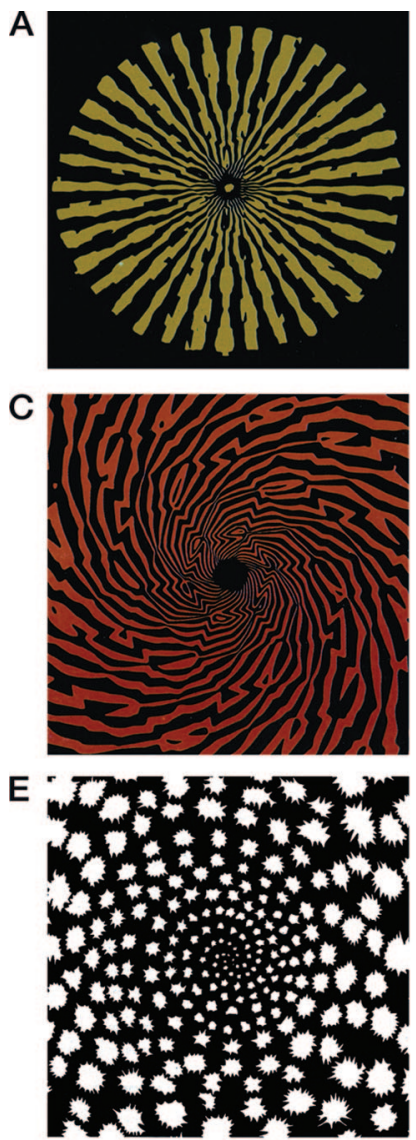

G

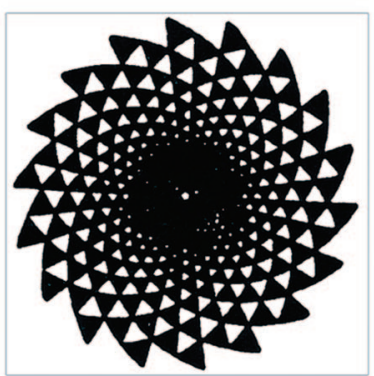

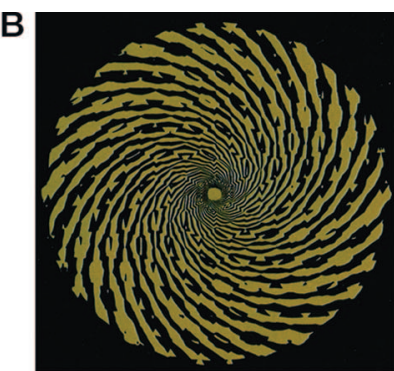
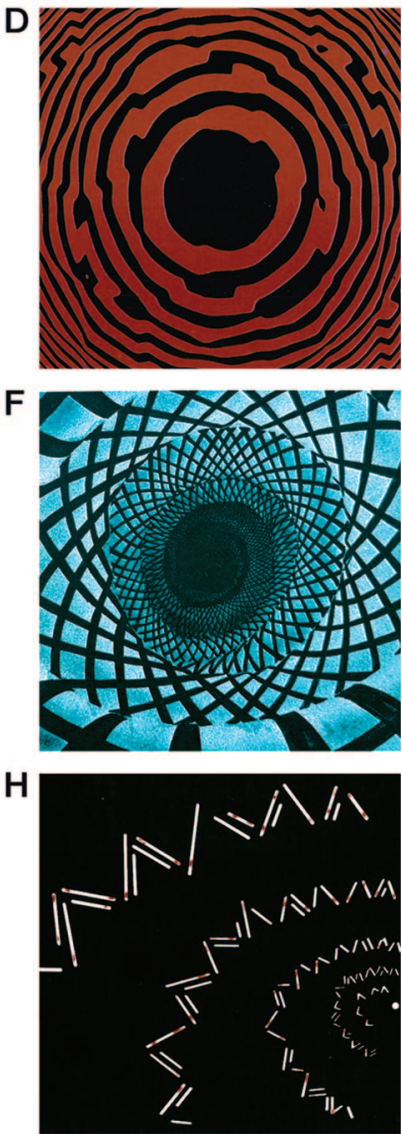

Figure 1. Some characteristic elementary visual hallucinations. A-D: These LSD flashbacks painted by Oster (1970) come in circular, radial and spiral geometries, three of the most common percepts cataloged by Klüver (1966) for many hallucinatory conditions. E: A proliferation of identical phosphenes (polyopia) induced by THC and arranged in a spiral geometry (Siegel \& Jarvik, 1975). F-G: Some more complicated lattice-like patterns produced by THC intoxication (Siegel \& Jarvik, 1975) and by binocular pressure on the eyes (Tyler, 1978). H: Superposition of fortification patterns produced by migraine; actual patterns flash and move across retina (Richards, 1971). Panels A-D from "Phosphenes," by G. Oster, 1970, Scientific American, 222(2), p. 82. Reprinted with permission. Copyright 1970 Scientific American, a division of Nature America, Inc. All rights reserved. Panels E-F from "Drug-Induced Hallucinations in Animals and Man," by R. K. Siegel and M. E. Jarvik, in R. K. Siegel and L. J. West (Eds.), Hallucinations (pp. 117 \& unnumbered page [Color Plate 6] following p. 146), 1975, New York, NY: John Wiley \& Sons. Copyright 1975 by John Wiley \& Sons. Reprinted with permission. Panel G from "Some New Entopic Phenomena," by C. W. Tyler, 1978, Vision Research, 18, p. 1637. Copyright 1978, with permission from Elsevier. Panel H from "The Fortification Illusions of Migraines," by W. Richards, 1971, Scientific American, 224(5), p. 90. Copyright 1971 by W. Richards. Reprinted with permission.

model describes the overall changes in arc shape and apparent speed as it propagates across cortex; this behavior is generic for weakly excitable media and can be mimicked by reaction-diffusion models (Dahlem \& Hadjikhani, 2009). A temporary scotoma (blind region) is left in the wake of the fortification arc's movement. Mapping the serrated arcs to cortical coordinates reveals that each serration covers about $1 \mathrm{~mm}$ of cortex and that the arc moves at a constant and rather stately speed of $2-3 \mathrm{~mm} / \mathrm{min}$ on cortex (Grüsser, 1995; Lashley, 1941; Richards, 1971; Wilkinson, Feindel, \& Grivell, 1999), requiring 20-25 min to traverse one entire side of striate cortex.

Richards (1971) suggested that the angularity of fortifications was consistent with activation of hexagonally packed orientation hypercolumns as a trigger wave swept through them (see Figure 2). The packing that Richards predicted is strikingly similar to the cortical iso-orientation pinwheel organization ultimately revealed by neuroanatomy (Bonhoeffer \& Grinvald, 1991; Swindale, Matsubara, \& Cynader, 1987). For other developments in fortification models, see Dahlem, Engelman, Löwel, and Müller (2000); Reggia and Montgomery (1996); and Schwartz (1980). When the psychophysics of migraine are compared to the topographical mapping qualities of visual cortices, the likely cortical loci of migraine percepts are Areas V1, V3a, and V8. Jagged arcs are consistent with the orientation processing in $\mathrm{V} 1$, but there is no reason to assume that other visual areas cannot be activated during migraine, and if activated, there is no reason to assume that this activity could not affect V1 via feedback. (Indeed, based on studies of cortical spreading depression, the condition could spread over the entire occipital lobe of the affected hemisphere but could have difficulty crossing prominent fissures between cortical areas, like the parieto-occipital sulcus.) Sacks (1995a) reported a range of phenomena consistent with the activation of many sensory areas. Interestingly, Hadjikhani et al. (2001) had a subject with an unusual exercise-induced aura-a drifting crescent-shaped cloud of TV-like noise-shown by functional magnetic resonance imaging (fMRI) to originate in V3a. (This percept resembled the twinkling textures induced adjacent to a centrally viewed patch of TV noise; Tyler \& Hardage, 1998.) Hadjikhani et al. suggested that classic fortification illusions may arise in V1 and color effects in V8. Functional imaging also shows cortical thickening abnormalities in areas V3a and MT of the brains of migraineurs, which is interesting because MT is important in motion perception and migraineurs are especially susceptible to visual motion-induced sickness (Granziera, DaSilva, Snyder, Tuch, \& Hadjikhani, 2006).

The slow movement of the fortification arcs suggests a diffusive-triggering process. The closest physiological analogue to the spread of a migraine fortification arc (and its accompanying scotoma) is a wave of cortical spreading depression, triggered in animal preparations by an infusion of potassium. The depression aspect is a matter of temporal scale: Initially, the spreading wave of extracellular potassium renders affected neurons briefly hyperexcitable, but as potassium concentration increases, the neurons become so depolarized that further action is suppressed for a longer period. In humans, Wilkinson (2004) suggested that a "wavefront of neural excitation operating on intrinsic cortical networks is presumed to underlie the positive hallucinations and the subsequent neuronal depression, the scotoma" (p. 308). Hadjikhani et al. (2001) found eight aspects of fMRI imagery during migraine corresponding to known aspects of cortical 
Table 1

Conditions That Trigger Hallucinations and the Nature of the Resulting Hallucinations

\begin{tabular}{|c|c|c|c|c|}
\hline Condition & Geometric pattern & Fortification & Phosphene & Complex \\
\hline Drugs & $\mathrm{O}, \mathrm{C}, \mathrm{D}$ & $\mathrm{S}^{\mathrm{a}}$ & S & $\mathrm{O}, \mathrm{C}, \mathrm{D}$ \\
\hline Photopic & $\mathrm{O}, \mathrm{C}, \mathrm{D}$ & $\mathrm{V}^{\mathrm{a}}$ & $\mathrm{S}, \mathrm{C}$ & $\mathrm{V}^{\mathrm{b}}$ \\
\hline Ocular pressure & $\mathrm{O}, \mathrm{C}, \mathrm{D}$ & & $\mathrm{O}$ & \\
\hline Migraine & $\mathrm{S}, \mathrm{C}$ & $\mathrm{O}, \mathrm{C}, \mathrm{D}$ & $\mathrm{S}, \mathrm{C}$ & $\mathrm{S}$ \\
\hline Epilepsy & S, C & $\mathrm{S}^{\mathrm{a}}$ & S, C, D & S \\
\hline Hypoglycemia & $\mathrm{S}$ & $\mathrm{S}^{\mathrm{a}}$ & & \\
\hline Transcranial magnetic stimulation ${ }^{\mathrm{c}}$ & $\mathrm{O}, \mathrm{C}, \mathrm{D}$ & & $\mathrm{O}$ & \\
\hline Electrical $(\text { cortex })^{\mathrm{d}}$ & S, P & & $\mathrm{O}, \mathrm{D}$ & $\mathrm{S}^{\mathrm{e}}$ \\
\hline Electrical (retina) ${ }^{\mathrm{f}}$ & $\mathrm{C}$ & & $\mathrm{O}$ & \\
\hline Charles Bonnet syndrome & $\mathrm{O}$ & & $\mathrm{O}$ & $\mathrm{O}, \mathrm{C}$ \\
\hline Sensory deprivation & $\mathrm{O}$ & & $\mathrm{O}$ & $S$ \\
\hline
\end{tabular}

Note. $\mathrm{O}=$ often; $\mathrm{S}=$ sometimes; $\mathrm{V}=$ very seldom; $\mathrm{C}=$ colored; $\mathrm{D}=$ dynamic; $\mathrm{P}=$ pinwheel (usually small and rotating).

a Sometimes a serrated form resembling a fortification illusion occurs but is usually stationary and usually does not flash. ${ }^{\mathrm{b}}$ If only one eye is stimulated by flicker while the other eye is kept dark, binocular rivalry between the eyes leads to switching between the geometric hallucinations and dark-phase hallucinations, which are swirling amorphous structures (Smythies, 1959a). ${ }^{\mathrm{c}}$ Noninvasive focal electrical stimulation of neural tissue, by induction, using a temporally alternating, spatially focused magnetic field. ${ }^{\mathrm{d}}$ Usually done with implanted electrodes as part of visual prosthesis studies, or during surgery. ${ }^{\mathrm{e}}$ Depends on which cortical area is stimulated. $\quad{ }^{\mathrm{f}}$ Usually done with current applied to entire orb.

spreading depression. One discrepancy is that the symptoms of migraine normally do not spread as far as the cortical spreading wave, suggesting that the strength of the wave falls below an activation threshold in the unaffected region (Dahlem \& Hadjikhani, 2009).

Since the first neural effect of cortical spreading depression is thought to be transient excitation, it is interesting that a variety of evidence suggests that the brains of migraineurs with aura are chronically hyperexcitable between attacks (Aurora \& Wilkinson, 2007). For example, thresholds for phosphene generation by TMS are reduced in subjects who experience migraine aura (Aurora, Ahmad, Welch, Bhardhwaj, \& Ramadan, 1998; Fierro et al., 2003; Mulleners, Chronicle, Palmer, Koehler, \& Vredeveld, 2001; Mulleners, Chronicle, Vredeveld, \& Koehler, 2002). Psychophysical evidence consistent with hyperexcitability or hypo-inhibition has been adduced by Chadaide et al., 2007; Chronicle, Wilkins, and Coleston (1995); Mulleners, Aurora, et al. (2001); Palmer, Chronicle, Rolan, and Mulleners (2000); and Wilkinson, Karanovic, and Wilson (2008).

\section{Epilepsy}

In epilepsy, like migraine, many unusual events are possible, depending on the cortical locus of the seizure. Compared to migraine, epileptic hallucinations are brief, with typical durations of several seconds rather than many minutes (a strong diagnostic discriminator between migraine aura and petit mal occipital epilepsy; Panayiotopoulos, 1999). This is consistent with electrophysiological findings that epileptiform activity is orders of magnitude faster than the $3 \mathrm{~mm} / \mathrm{min}$ cortical spread of migraine (Chervin, Pierce, \& Connors, 1988; Penfield \& Rasmussen, 1950). The percentage of epilepsy cases with visual symptoms (about $11 \%$ in Penfield \& Kristiansen, 1951) seems surprisingly small (given the share of cortex involved in some ways with vision); symptoms range from simple phosphenes to the complicated scenic hallucinations of temporal lobe epilepsy (Fried, Spenser, \& Spenser,
1995; Penfield \& Kristiansen, 1951; Wilkinson, 2004; Williamson, Thadani, Darcey, Spenser, \& Mattson, 1992). Phosphenes have a variety of simple shapes (stars, streaks, spots, annuli, wedges, and occasionally a zigzag like a piece of a fortification illusion) and may be strongly colored. In occipital epilepsy, circular phosphenes and annular forms (e.g., doughnut phosphenes) seem particularly prevalent (Panayiotopoulos, 1999). Single-wedge and doublewedge (butterfly or bow-tie) phosphenes are also seen. Wilkinson's (2004) review found that "components may be stationary and long-lasting, but more commonly flicker, pulsate, twinkle or move. Several patterns of motion (translational, rotary, expansion, contraction and random) have been described" (p. 311). If a subject has a characteristic epileptic aura, it can often be triggered by focal electrical stimulation during neurosurgery (Penfield \& Rasmussen, 1950). Occipital epilepsy and its associated hallucinations can be controlled by medicines that dampen neuronal excitability (e.g., carbamazepine; Panayiotopoulos, 1999).

\section{Ocular-Pressure-Induced Forms}

It has been known since antiquity that illusory images are induced by applying pressure to the eyeballs; a light spot (phosphene) appears opposite the point of pressure (if localized) and can be seen in the dark. Some believed that ocular pressure created internal light, but Descartes and Newton correctly anticipated that pressure mechanically stimulates the retina (Grüsser \& Hagner, 1990; Wade \& Brozek, 2001). Phosphenes can sometimes be seen during eye movements and accommodation in the dark, or when ocular pressure is abruptly raised (e.g., sometimes by coughing; Grüsser \& Landis, 1991). The immediate retinal effect of ocular pressure is to activate retinal on-center ganglion cells (after a delay of $0.2 \mathrm{~s}$ ) and inhibit off-center ganglion cells, resulting in perception of a light increment (Grüsser, Grüsser-Cornhels, Kusel, \& Przybyzewski, 1989; Grüsser, Hagner, \& Przybyswewski, 1989). Grüsser and colleagues (Grüsser, Grüsser-Cornhels, et al., 1989; Grüsser, Hagner, \& Przybyswewski, 1989) theorized that defor- 


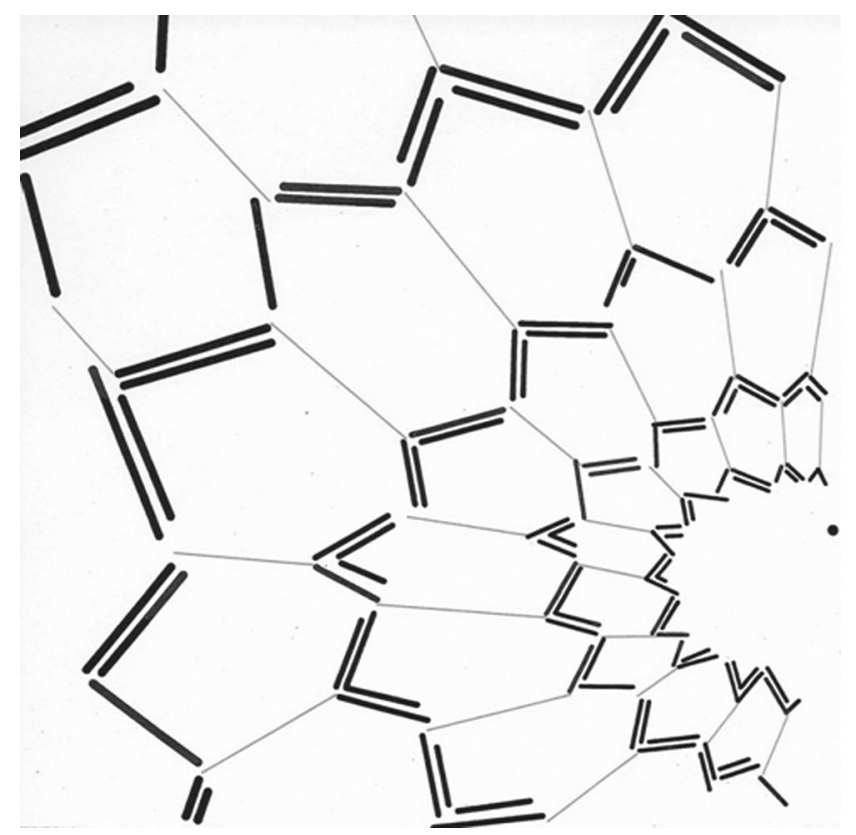

Figure 2. An interesting interpretation of a migraine attack like that depicted in Figure 1H (a superposition of several migraine fortification patterns seen by one subject during the course of an attack). Each vertical arc in Figure 1H represents one snapshot of the illusion. Here, Richards (1971) has connected features from successive fortification patterns, revealing a hexagonal-like pattern of underlying activity that closely resembles the hexagonal packing of cortical orientation hypercolumns revealed by neuroanatomy. From "The Fortification Illusions of Migraines," by W. Richards, 1971, Scientific American, 224(5), p. 93. Reprinted with permission. Copyright 1971 Scientific American, a division of Nature America, Inc. All rights reserved.

mation stretches the horizontal cell layer, depolarizing on-center bipolar cells and hyperpolarizing off-center cells. Continued deep ocular pressure (for more than about $40 \mathrm{~s}$ ) results in a temporary blindness via ischemia (like applying a choke hold directly to the retina) - a useful technique often employed by psychophysicists. In the time between phosphene induction and eventual blindness lies an interesting perceptual phenomenon: Sustained simultaneous binocular pressure evokes complicated Klüver-like percepts, like the lattice spiral in Figure 1G (Tyler, 1978). The requirement for binocular stimulation is intriguing; the instructive exception is that for some amblyopic subjects, stimulation of the good eye is sufficient to induce geometric forms (Grüsser \& Landis, 1991.) Most pressure percepts are not consistent with the entopic (intrinsic anatomical) retinal structures posited by Purkinje (1819/1823), and their commonality with other geometric hallucinations suggests that pressure-induced excitation drives a cortical pattern-forming mechanism.

\section{Electrical Stimulation of Retina}

After Galvani's dramatic demonstrations of electrically induced muscle contraction and Volta's invention of the battery, it was not long until electric current was applied to the head and especially the eye. As for so many visual effects, Jan Purkinje (1819/1823) led the way. Light sensations were generally re- ported at onset and offset of current. Some studies placed one electrode on the roof of the mouth and the other on the forehead; others applied current directly to the eye, sometimes immersing the eyes in salt water and passing the current though the solution. Vivid colors, phosphenes, and patterns were produced in these experiments. At high frequencies, large colored crescent, horseshoe, smoke-ring and ameba-shaped phosphenes appear, which persist to stimulation rates of $100 \mathrm{~Hz}$ in the dark and up to $210 \mathrm{~Hz}$ in light-adapted eyes (Wolff, Delacour, Carpenter, \& Brindley 1968); the retina can follow higher rates with electricity than light, in part because the photochemical bottleneck in the photoreceptors is bypassed. Wolff et al. (1968) found that geometric patterns seen for $10-40 \mathrm{~Hz}$ sinusoidal electrical stimulation were equivalent to those seen for flickering light. There is an understated heroic quality to many of these early experiments, which mostly used the investigators as subjects. Brindley (1955) noted that his experiment, which applied the electrodes directly to the conjunctiva of the eye, required topical anesthesia; despite this, the experiment was difficult because the current through his anesthetized eye spread to other nerves and made his teeth ache severely.

Alternately, electric retinal stimulation can produce effects unique to retinal dynamics. Carpenter (1973) electrically stimulated the eye with $100 \mathrm{~Hz} \mathrm{AC}$ current and found that if a dark object passes through the visual field, a series of thin light illusory contours form in the passing object's wake. These contours obey four rules: (a) The illusory contours disappear promptly when the current is turned off. (b) One contour forms for each cycle of the alternating current completed while the moving object transits the visual space. (c) Half of the contours move with the wake; the other half move against it. (d) If two contours collide, they neither pass through one another nor mutually annihilate, but rather, they merge to form loop-like structures. Drover and Ermentrout (2006) modeled the formation of such contours by populations of gapcoupled neurons responding to every other cycle of the driving frequency: Some contours are generated by even-numbered stimulus cycles and others generated by odd-cycles, each with a different motion bias as they form traveling waves. These wake contours are reminiscent of Charpentier's bands (McDougall, 1904): a series of three to four afterimages produced over about 50 ms when a bright bar is moved in the dark. This periodicity corresponds to a sampling rate of at least $60 \mathrm{~Hz}$. Drover and Ermentrout's half-sampling mechanism for wake contours, if applied to the $120 \mathrm{~Hz}$ oscillations found in the electroretinogram (ERG; King-Smith, Loffing, \& Jones, 1986), might account for Charpentier's bands as well.

\section{Electric Stimulation of Cortex (Including TMS)}

High voltages applied to the scalp near visual cortex produce diffuse cortical stimulation, creating textured phosphenes several degrees across (Merton \& Morton, 1980). Knoll, Kugler, Eichmeier, and Höffer (1962) used temporal electrodes driven by $5-100 \mathrm{~Hz}$ electric current to induce geometric patterns in 46 observers similar to patterns seen in flickering lights and in direct electric cortical stimulation experiments (Penfield \& Rasmussen, 1950). Many of Knoll et al.'s subjects saw the hallucinatory patterns better when light-adapted, a fact noted repeatedly for other hallucinatory inducers. Eichmeier and Nie- 
dermaier (1976) found that it was easier to electrically stimulate geometric hallucinations at high altitudes, presumably as a side effect of hypoxia. This is interesting because hypoxia also facilitates triggering the cortical spreading depression phenomenon linked to migraine (Dahlem \& Müller, 2004; Grafstein, 1963; Leão, 1963). Some transcranial electric stimuli may also inadvertently excite the eye (Motokawa, 1970; Paulus, 2010); the persistence of hallucinations after temporarily pressureblinding the eyes is a useful check.

Direct electric stimulation of the human cortex (V1-V3) was used by Penfield and Rasmussen (1950) while locating surgically excisable epileptic foci. Stimulation of V1 usually leads to small colored phosphenes. Stimulation outside V1 often leads to moving colorless phosphenes. Pulse trains are more effective than single stimuli or direct current, and phosphene thresholds are smallest in cortical layers 4-6 (Bak et al., 1990). Pulse trains longer than about $15 \mathrm{~s}$ become ineffective (Dobelle, Mladejovsky, Evans, Roberts, \& Girvin, 1976; Dobelle, Quest, Autunes, Roberts, \& Girvin, 1979). Electrode separations of 1 $\mathrm{mm}$ or less result in a single phosphene (Bak et al., 1990), consistent with estimates of human cortical column size. Surface electrodes tend to induce flicker, while deeper electrodes tend to induce steady deeply colored circular phosphenes. Increasing stimulus strength raises the phosphene's brightness while reducing its size (Bak et al., 1990, and Evans, Gordon, Abramov, Mladejovsky, \& Dobelle, 1979, found a logarithmic relation for phosphene brightness). Brindley and Lewin (1968) used arrays of phosphene-inducing electrodes while developing a neural prosthetic for the blind. Maps of phosphene size as a function of electrode position can be used to estimate cortical magnification: Phosphenes generated in central vision are punctuate, while phosphenes in peripheral vision may be several degrees wide (Dobelle et al., 1976, 1979).

TMS is a special case, intermediate to transcranial electric stimulation and direct cortical stimulation using small electrodes. An alternating magnetic field-applied across the skull-induces electric currents in the cortex. Unlike transcranial electric stimulation, TMS stimuli are generally painless. Special antennas localize stimulation to several centimeters of cortical area, which, although not as precise as those induced by direct cortical electric stimulation, is more convenient; based on other induced hallucinations, the increased spatial extent of stimulation is likely to bias the system to geometric hallucinations rather than simple phosphenes. In Marg and Rudiak (1994) and Kammer, Puls, Erb, and Grodd (2005), the most common percepts were wedges and butterfly patterns (double wedges); grids and hexagonal lattice patterns were also reported. Single pulses of TMS do not produce illusory features in inexperienced observers (Kammer et al., 2005), but short trains of stimulation (two to five pulses) reliably produce hallucinations in all observers (Boroojerdi et al., 2002; Ray, Meador, Epstein, Loring, \& Day, 1998). The position and shape of the phosphene in Krammer et al.'s experiment did not vary much with the skull position of the coil over occipital cortex; to explain this invariance, Kammer et al. speculated that their TMS activated cortex indirectly, via the optic radiations to V1 and back-projecting fibers from V2 and V3 onto V1.

\section{Flicker-Induced Hallucinations}

Flicker-induced hallucinations were discovered by Purkinje (1819/1823) and Brewster (1834). Purkinje evoked colored patterns while waving his spread fingers between a bright light and his shut eyes. Brewster (the inventor of the kaleidoscope) saw illusory colored patterns while rushing past a sunlit fence with his eyes shut - a result enjoyably replicable for passengers on fast drives through sunlit forests (Billock \& Tsou, 2010). Both cases illustrate the optimal stimulus for flicker-induced hallucinations - a strong source of spatially homogeneous temporal modulation (closed eyes are relatively uniform diffusers). Later studies by Fechner (1838) and others used rotating black-and-white sector disks: At a sufficiently high rotation rate, the individual rotating sectors fuse into a uniform flickering gray. Many observers see hexagonal pastel-colored patterns on the disk under these circumstances, but other patterns are reported as well. Most modern studies of flicker-induced hallucinations use stroboscopic illumination of a uniform surface (a ganzfeld) or the flickering empty screen of a CRT. In clinical studies, illusory forms and colors are a frequently reported side effect of flicker ERG/electroencephalogram (EEG) tests. W. G. Walter (1956) stimulated thousands of patients with stroboscopic-illuminated ganzfelds, all of whom reported illusory colors, movements, and patterns; the optimum frequency for evoking the effects varied greatly between observers, and the most common patterns were moving concentric rings and spoke patterns. Mundy-Castle (1953) made the same claims for his 1,000 subjects. Many studies find that flicker rates of about 4-25 Hz elicit illusory color and forms (Brown \& Gebhard, 1948; Freedman \& Marks, 1965; Mundy-Castle, 1953; Remole, 1971, 1973; Smythies, 1959a, 1959b, 1960; V. J. Walter \& Walter, 1949).

In our experience, hallucinations do occur at low flicker rates but are more salient at moderate rates (ca. 10-20 Hz). The form of the hallucinated pattern may be frequency dependent (e.g., Allefeld, Pütz, Kastner, \& Wackermann, 2011; Becker \& Elliot, 2006; Young, Cole, Gamble, \& Rayner, 1975). There seems to be little consistency between studies, possibly due to differences in stimulation techniques. The most ambitious attempt to determine frequency dependency is Allefeld et al.'s (2011) strobed-ganzfeld study. They plotted frequency-of-seeing distributions for about 3,000 occurrences of 17 different spatial percepts and found that they occur mostly in the 5-26 Hz range. Several of their 17 perceptual patterns correspond to Klüver's forms (and ErmentroutCowan cortical stripe formation). Both spirals and concentric circles (ripples) have perceived frequency distributions that peak at about $15-18 \mathrm{~Hz}$. Three separate categories correspond to radial hallucinations (wheel, sun, star) and have peaks between about 7 and $18 \mathrm{~Hz}$. Hexagonal (honeycomb) and rectilinear (raster) lattices have peaks near 15 and $30 \mathrm{~Hz}$, respectively. Phosphene-like illusions (spot, organic) are more common at 25-30 Hz. Color in these hallucinations is most common at $11-18 \mathrm{~Hz}$ (much higher than for Fechner-Benham illusory colors, which are most vivid for flicker rates near $6 \mathrm{~Hz})$.

Remole $(1971,1973)$ measured luminance contrast thresholds for flicker-induced illusory geometric patterns as a function of temporal frequency; some of these tuning functions resemble resonance curves. For binocular vision, his subjects had lower thresholds for flash rates of 10-18 Hz. For monoc- 
ular vision, thresholds were higher, and the function's minimum was (for two thirds of observers) shifted to higher frequencies. Slightly lower thresholds for binocular vision are expected on information theoretic grounds (by a factor of $\sqrt{2}$ ), which contrasts with the near absolute requirement of binocular pressure for ocular pressure hallucinations. Remole's results lie mostly between these extremes: At some frequencies, binocular thresholds are an order of magnitude lower than for monocular vision, while at other frequencies, the binocular advantage drastically narrows. Remole also examined wavelength effects: Flickering yellows and reds required less luminance than blues and greens to induce hallucinations, and the threshold differences for red versus green and blue versus yellow were highest at about 14 $\mathrm{Hz}$; Remole believed this implicates color opponency in hallucinatory pattern formation. Reports vary on the qualia of colored hallucinations, with some subjects finding the colors unearthly (W. G. Walter, 1956) and either too vivid or not vivid at all (we have found pastel colors in our experiments, like those induced in Fechner-Benham subjective color; Billock \& Tsou, 2006). As W. G. Walter (1956) pointed out, on some level the colors are real enough: Brady (1954) conditioned subjects to give a galvanic skin response to red lights and found that flicker-induced red led to the conditioned response.

Although W. G. Walter (1956) made much of the stroboscope's very short pulse width, Smythies (1960) tested various waveforms and light-to-dark pulse widths and concluded that the waveform's exact shape has little effect, if temporal alteration of light and dark is vigorous. In a foreshadowing of experiments using visual noise (Billock \& Tsou, 2007; MacKay, 1965), Smythies (1960) noted that random flicker also works well. Smythies reported that sudden changes in the flicker frequency while hallucinating can cause the hallucinated features to become finer/more numerous with higher temporal frequency and coarser/less numerous with lower temporal frequency. Smythies (1960) also tried stimulating the two eyes with out-of-phase flicker and noted that the perceptual effects were similar to doubling the frequency of stimulation. Given Remole's (1973) results, it would be interesting to know what effect this has on the pattern-formation thresholds. When only one eye is stimulated, hallucinations vary with the state of binocular rivalry. Light-phase hallucinations (the geometric percepts addressed throughout this article) occur during rivalry periods when the stimulated eye is dominant, while dark-phase percepts (swirling amorphous patterns, boiling, ameba-like blobs, etc.) occur when the patched eye is dominant (Brown \& Gebhard, 1948; Smythies, 1959a). Smythies (1959a) argued that the patterns are visualizations of the noise in the patched eye. This is supported by one study of spatiotemporal fractal noise, which gave rise to percepts of similar character (Billock, Cunningham, Havig, \& Tsou, 2001). They also resemble some of the smoke-like contours reported for some apparent-motioninduced hallucinations (Billock \& Tsou, 2007) and for some noise stimuli (Fiorentini \& MacKay, 1965; MacKay, 1965).

\section{Other Photopic-Induced Hallucinations}

Motion-induced hallucination. There are at least two other kinds of photopically induced patterns: motion-induced hallucinations and MacKay effects. Among motion-induced illusions are the illusory and supernumerary spokes reported by Tynan and Sekular (1975); Holcombe, Macknik, Intrilgator, Seiffert, and Tse (1999); and Purves, Paydarfar, and Andrews (1996). A particularly spectacular motion-induced pattern-forming effect was discovered by Mayzner (1975), while manipulating the marquee light illusion. Mayzner linked flashing lights into a closed circular or square circuit (see, e.g., Figure 3); the flashing icons appear to move about the circuit, taking corners like ducks in a shooting gallery. When four or five equally spaced illusory-moving lights are viewed in the dark, the imaginary space defined by the movement fills with illusory colors and rotating shapes. The two studies to address this phenomenon only differ in the nature of the color. Mayzner described a succession of vibrant colors filling the space. Billock and Tsou (2007) found smoky swirls of more pastel colors, often green, purple, and gray, interlaced into a pulled-taffy-like texture. This texture evolves into a storm-like pattern, with gray arms reaching out toward the icons. The arms straighten out into a

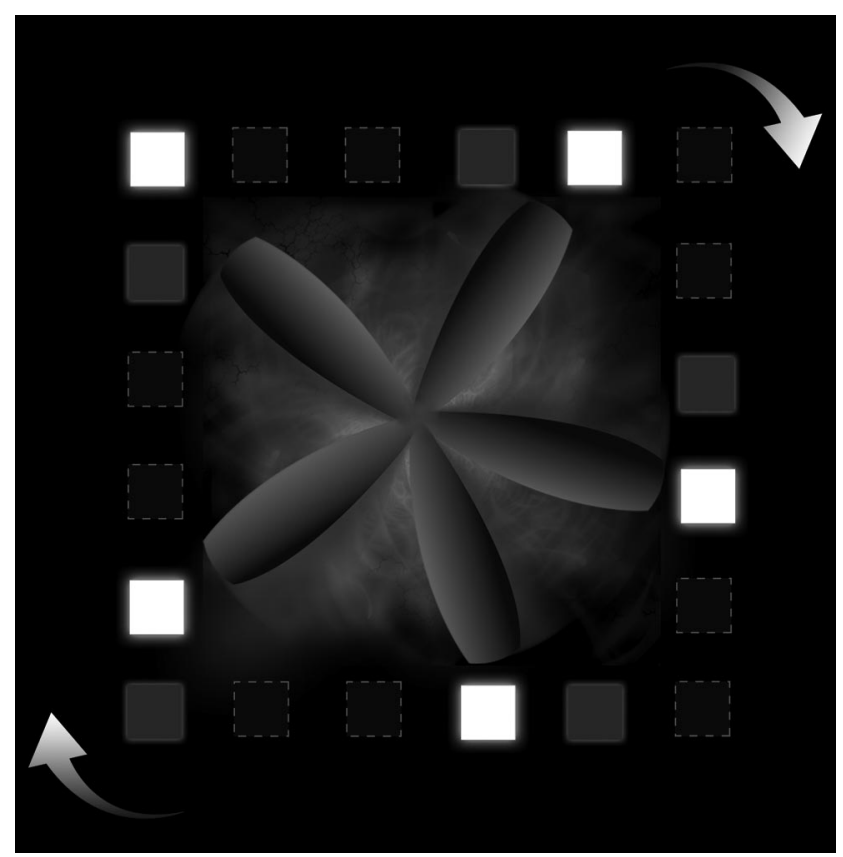

Figure 3. An artistic rendition of a Mayzner illusion induced by illusory motion. Illusory motion is induced by turning icons on and off in turn (the marquee light illusion). Here, a square is defined by 20 such icons. If every fourth icon is lit and then extinguished, followed by the icons clockwise to them, and so on, the percept is of five icons moving around an imaginary square. Active icons are depicted in white, newly extinguished icons (still faintly visible due to visual persistence) are depicted in gray, and other icons are outlined in dashes. When viewed in the dark, the center of the square fills with a smoky or taffy-like colored texture that resembles a storm pattern. During viewing, radial arms appear in the storm pattern and reach out toward the moving icons, forming a five-bladed hallucinatory propeller. However, the number of blades may be as great as the number of visible icons (both the active icons and the ones visible because of visual persistence). From "Neural Interactions Between Flicker-Induced SelfOrganized Visual Hallucinations and Physical Stimuli," by V. A. Billock and B. H. Tsou, 2007, PNAS: Proceedings of the National Academy of Sciences, USA, 104, p. 8493. Copyright 2007 National Academy of Sciences, USA. Reprinted with permission. 
propeller shape rotating in time with the illusory movements of the flashing icons (see Figure 3).

MacKay effects. Donald MacKay (1957a, 1957b, 1965, 1978) described a series of phenomena that have become known as MacKay effects, without much separate consideration of their diverse nature. Most of these effects involve some kind of opponency between certain spatial forms. If a pattern of concentric circles is steadily fixed for many seconds and then replaced with a blank field, a faint noisy fan-like shape can be seen as an afterimage. Similarly, if the same pattern is viewed in flickering light in an otherwise dark room, a fan-shaped pattern will be seen superimposed on the concentric circles. MacKay treated fan shapes and concentric circles as complementary (opponent forms) in sequential contrast with one another and interpreted the effects of flickering light as two phases of perception superimposed in one another: The physical concentric circles are seen during the flashes, and their complementary afterimage is seen during the dark phase. Similar superimposed patterns can be seen while viewing closely spaced patterns under steady room light. Here, a flicker-like temporal stimulation is supplied by eye movements across the closely spaced features. This interpretation is reinforced by the absence of this third form of the MacKay's hallucinatory effect under retinal image stabilization. Another variation of the MacKay effect was seen when the physical patterns were printed on transparencies and illuminated by dynamic spatial noise (e.g., TV static) from behind. MacKay reported two forms of motion for this stimulation: one that seems to flow along the transparencies' contours and one that seems to flow at right angles to those contours. The latter is like a MacKay opponent effect, but actual illusory contours are weak or absent. The strengths of the two effects seem to vary with the channel the TV noise is taken from, suggesting that the statistics of the noise matter. Billock and Tsou (2007) were able to strengthen the MacKay noise effect using fractal noise; when viewed through concentric circles, hallucinatory rotating fan shapes are seen; when viewed through fan-shape patterns, pulsating hallucinatory circles are seen. Finally, MacKay (1965) and Fiorentini and MacKay (1965) noted that noise can aggregate into moving shapes under certain conditions: If frames of spatial noise are interspaced with frames of uniform luminance, the noise pixels seem to aggregate into maggot-like wriggling forms. The effect is strongest in binocular vision and does not occur if the noise is viewed by one eye and the blank frames by the other eye.

\section{Drug-Induced Hallucinations}

Hallucinations seen in the early stage of drug intoxications range from simple phosphenes to patterns as complicated as intricate Persian rugs. Klüver (1966) compared reports on drug-based hallucinations and found that most hallucinations fit into four basic categories: (a) lattices (rhomboidal and hexagonal), (b) cobwebs, (c) radial and concentric forms (fan shapes and bull's-eye-like patterns) whose three-dimensional versions resemble tunnels and funnels, and (d) spirals. Figures $1 \mathrm{~A}-1 \mathrm{G}$ show a variety of these Klüver geometries. Klüver also noted that these same forms can be seen under many other conditions. Drug hallucinations are often reported to be very bright (similar in this respect to migraine fortification patterns) and vividly colored (in contrast to the pastel colors usually seen in flicker-induced hallucinations). Paintings and drawings of these vivid images show that the same forms are seen by independent observers across different cultures (Siegel \& Jarvik, 1975). These drawings also provide evidence of a characteristic spatial scale (roughly $2 \mathrm{~mm}$ on cortex) for drug hallucinations, estimated by comparing the visual cortex's dimensions to the number of illusory periodic features. Hallucinogenic drugs generally modulate one of four neurotransmitter-based mechanisms: acetylcholine (e.g., scopolamine), dopamine (amphetamine, cocaine), glutamate (ketamine, PCP) or serotonin (LSD, mescaline, psilocybin). For a useful recent review of hallucinogens and neurotransmitters, see Aleman and Larøi (2008). Not surprisingly, clinical conditions that affect these neurotransmitters can also induce hallucinations; for example, about $25 \%$ of patients with Parkinson's disease (a dopamine defect; Wolters \& Berendse, 2001) report hallucinations, as do $60 \%$ of patients with Lewy body dementia (an acetylcholine defect; Aleman \& Larøi, 2008). As in epilepsy, the locations of neural defects and lesions matters: Lewy body dementia patients who have visual hallucinations tend to form more Lewy bodies in visually specialized areas of temporal lobe than patients who are hallucination free (Harding, Broe, \& Halliday, 2002).

\section{Sensory Deprivation and Charles Bonnet Syndrome}

In the 1950s, Donald Hebb was interested in the effects of conditions rumored to have been employed in brainwashing and coerced confessions (Heron, 1961). Students in his lab discovered that systematically depriving the brain of differentiated sensory inputs could lead to hallucination (Bexton, Heron, \& Scott, 1954; Heron, Doane, \& Scott, 1956). For example Heron et al.'s (1956) subjects wore translucent goggles to provide spatially unstructured stimulation, had audition masked by ventilation fan noise, and wore cardboard sleeves to limit tactile stimulation. All 14 subjects reported visual hallucinations - at first phosphenes and geometric forms but later some more complex hallucinations as well (the same evolution reported for hallucinogenic drugs). Interestingly, studies in which the subjects were in darkness reported fewer hallucinations, and more time was often required before hallucinatory onset (cf. Vernon, 1963; Zubek, 1969), but other differences between studies may have influenced the incidence of hallucination (Zuckerman \& Cohen, 1964). Sometimes, these sensory deprivation effects occurred as a side effect: About $39 \%$ of directors of medically protective environments like laminar flow units (for treatment and prevention of infection) reported hallucinations in their patients - the fourth most common side effect in this study (Kellerman, Rigler, \& Siegel, 1977). Nature provides a clinical version of a tightly controlled sensory deprivation experimentCharles Bonnet syndrome (CBS) — in which subjects who have visual losses experience both simple and complex visual hallucinations (ffytche, 2005; Wilkinson, 2004). In the literature, the complex illusions get more emphasis, but actually, the simple hallucinations seem to predominate. For example in Lepore's (1990) 59 patients with CBS, 63\% had only elementary hallucinations, and $27 \%$ had both elementary and complex hallucinations. Of the simple percepts, grids and lattices are common, especially for losses of central vision (e.g., macular degeneration). Functional imaging (fMRI) during these elementary hallucinations shows increased activity in occipital cortex (ffytche, Howard, Brammer, Woodruff, \& Williams, 1998); patients who hallucinate in color 
also show activity in the posterior fusiform gyrus, which is known to contain concentrations of color sensitive neurons. Bexton et al. (1954) recognized from the beginning that the hallucinations reported for sensory deprivation were probably related to CBS (although they did not use the term); they cited similar cases in the nondemented elderly with conditions like cataract. ffytche (2008) remarked that the percepts reported by his CBS patients resemble flicker-induced hallucinations.

\section{Geometric Hallucinations and Their Potential Neural Correlates}

Of the three kinds of elementary hallucinations, the geometric hallucinations described by Klüver (1966) are of special interest because they are ubiquitous in conditions that lead to any kind of elementary hallucination and because these shapes connect directly to certain kinds of neural pattern-forming mechanisms. Chief among these Klüver forms are lattice and cobweb-like structures, fan shapes, concentric circles, spirals, and related threedimensional structures like tunnels and funnels. Siegel and Jarvik (1975) proposed expanding Klüver's categories to include characteristic motions, but for our purposes, the actual forms are a good starting point; these Klüver form constants are fodder for neural theorists, and their characteristic motions and other dynamics emerge naturally from the theoretical treatment of the forms. Ermentrout and Cowan (1979) noted that spiral, fan-shaped, and circular Klüver hallucinations (see Figure 1) are all perceptual correlates of stripe patterns on visual cortex (see Figures 4 and 5). This stems from the nonlinear neural mapping of retina to cortex (see the Appendix): Parallel stripes of cortical activity are generated by viewing physical concentric circles, fan shapes, or spirals (these cortical stripes are seen with functional imaging; see, e.g., Figure 5). Conversely, if stripes of activity autonomously form on visual cortex, then their orientation on cortex determines the shape of the resulting hallucination (Ermentrout \& Cowan, 1979). Scrolling of the cortical stripes yields rotation for fans/spirals and inward/outward movement for concentric patterns. Rotation of the stripes yields morphing between the percepts; a radial form can twist into a spiral, which tightens until it is a set of concentric circles. Kaleidoscopic changes between Klüver-like patterns are seen under many conditions; Sacks (1995b) estimated that morphological changes can occur about 10 times per second during migraine. More than one set of stripe patterns can be generated at a time. Superposition of simple cortical activity patterns models more complicated lattice and cobweb hallucinations (Ermentrout \& Cowan, 1979). Competition between coevolving cortical stripe patterns may be another route to changes in perceived form.

Related stripe-like patterns arise in nature: the parallel cylindrical rolls of rising hot and falling cold fluid formed during fluid convection, the intricate patterns on seashells, the spot-and-stripe camouflage adorning many animal skins (see Figures 6, 7, and 8; Bestehorn \& Haken, 1991; Ermentrout, Campbell, \& Oster, 1986; Kondo \& Miura, 2010; Meinhardt, 2003; Murray, 1988; Turing, 1952). Nothing dictates the point-by-point behavior of these systems-self-organized patterns arise from nonlinear dynamic interactions of many neighboring units. To explain autonomous cortical stripe formation, Ermentrout and Cowan (1979) created an excitatory-inhibitory neuronal network; under some conditions, if excitation is uniformly increased above a critical level (either by
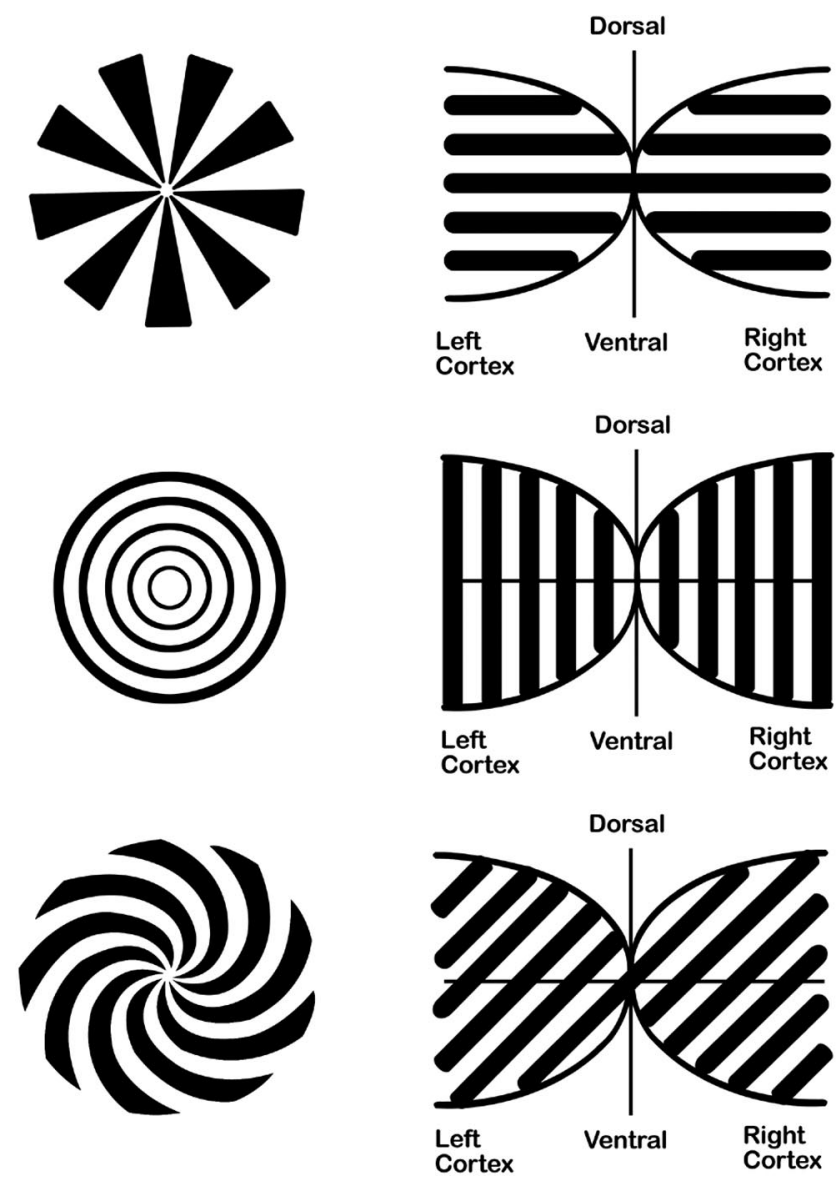

Figure 4. Mapping of retinal geometric patterns to cortical stripe patterns. Physical geometry imaged on retina (left) is mapped nonlinearly onto cortex (right), resulting in stripe patterns of neural activation on cortex. Conversely, if oriented stripes of neural activity form on cortex, they should evoke the corresponding hallucinatory percept on the left: the Ermentrout and Cowan (1979) thesis. Some more complicated percepts (e.g., polar webs, hexagonal lattices) can result from superposition of different cortical stripe patterns. From "Neural Interactions Between Flicker-Induced Self-Organized Visual Hallucinations and Physical Stimuli," by V. A. Billock and B. H. Tsou, 2007, PNAS: Proceedings of the National Academy of Sciences, USA, 104, p. 8491. Copyright $2007 \mathrm{Na}$ tional Academy of Sciences, USA. Reprinted with permission.

external stimuli or internal conditions), then this neuronal network generates parallel stripes of cortical activity. The orientation of this cortical pattern (and its perceptual correlate) is unpredictable and can be unstable.

\section{Basic Models of Self-Organized Hallucinatory Neural Pattern Formation}

Most models of cortical pattern formation build on the Ermentrout-Cowan model, a member of the class of WilsonCowan models used in a wide range of nonlinear dynamic neural and perceptual problems (Ermentrout \& Cowan, 1979; Wilson, 1999; Wilson \& Cowan, 1973). Like other spontaneous patternforming systems, there are two structural requirements: an asymmetry between two interacting mechanisms and a diffusion-like 


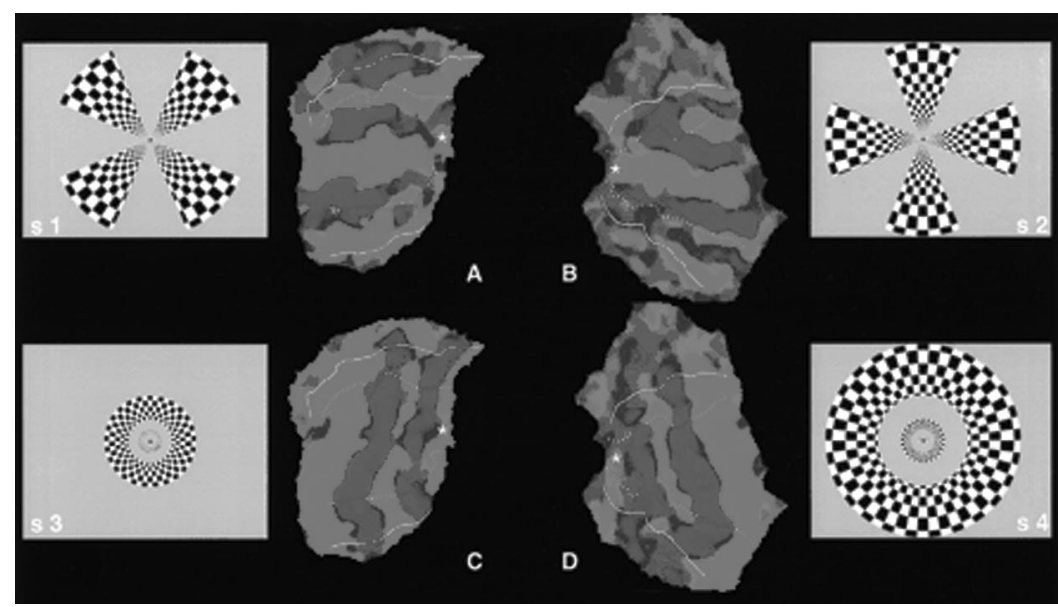

Figure 5. A physical demonstration of cortical stripes and their perceptual correlates. The nonlinear mapping from retina to primary visual cortex (V1) introduced in Figure 2 can be seen using functional imaging of human cortex (in this case, functional magnetic resonance imaging) while viewing physical patterns. A-B: Radial patterns on retina (s1, s2) evoke horizontal stripes on V1. C-D: Concentric patterns on retina (s3, s4) evoke vertical stripes on V1. The dynamic reversing checkerboard patterns shown to retina are used to force both onand off-cells (with both sustained and transient temporal properties) to respond nearly continuously, yielding a strong blood oxygen level-dependent response. From "Functional Analysis of Primary Visual Cortex (V1) in Humans," by R. B. H. Tootell et al., 1998, PNAS: Proceedings of the National Academy of Sciences, USA, 95, p. 813. Copyright 1998 National Academy of Sciences, USA. Reprinted with permission.

mechanism for spreading their influences. Both can manifest in subtle ways. For the cortex, the conceptually simplest model is a set of coupled integrodifferential equations, each pair of which represents dynamic neural interactions in one particular cortical location. ${ }^{3}$

$$
\begin{gathered}
\frac{\partial E}{\partial t}=-E+\mathrm{S}_{\mathrm{E}}\left\{a W_{E E} * E-b W_{I E} * I+\text { Sensory Input }\right\}, \\
\frac{\partial I}{\partial t}=-I+\mathrm{S}_{\mathrm{I}}\left\{c W_{E I} * E-d W_{I I} * I+\text { Sensory Input }\right\} .
\end{gathered}
$$

Here, $S_{E}$ and $S_{I}$ are sigmoidal neural response nonlinearities, and the $W * E$ and $W * I$ terms are spatial convolutions of neural activity with excitatory $(E)$ and inhibitory $(I)$ spatial weighting functions; for example, $W_{E E}(x, y){ }^{*} E(x, y)=\iint W_{E E}\left(\tau_{1}, \tau_{2}\right) E(x-$ $\left.\tau_{1}, y-\tau_{2}\right) d \tau_{1} d \tau_{2}\left(\tau_{1}\right.$ and $\tau_{2}$ are dummy integration variables). The neural interactions are asymmetric: Neighboring excitatory cells excite each other and inhibitory cells alike, while inhibitory cells inhibit both other inhibitory cells and excitatory cells (related subtle asymmetries lie at the heart of every pattern-forming system). The Gaussian-like neural-weighting functions $\left(W_{i, j}\right)$ describe the influence that neighboring neurons have on particular neurons as a function of distance; the resulting interplay between excitation and inhibition mimics the spatial derivative operator found in other pattern-forming systems. The range of cortical excitation-about 1 $\mathrm{mm}$ - is roughly the width of a human cortical orientation ocular dominance column (a fundamental unit of cortical organization), while inhibition can span several columns. This excitatory range matches the spatial scale seen in the migraine fortification hallucination - a broad flattened vertical arc made up of bright flickering line segments set at roughly $60^{\circ}$ angles to each other-when adjusted for retinotopic location, each segment is approximately
$1.2 \mathrm{~mm}$ long on cortex (Richards, 1971). Similarly, the hypercolumn spacing in human cortex (about $2 \mathrm{~mm}$; Horton, 1996; Horton $\&$ Hedley-White, 1984) roughly fits the maximum frequency of hallucinatory features (for a fan geometry) with about 15 features (and blank spaces between features) per cortical hemifield (in accord with the rough number of cortical hypercolumns that could be organized along the circa $60-\mathrm{mm}$ extent of each hemisphere's V1; Bressloff et al., 2001). (Each 2-mm-wide hypercolumn contains two 1-mm orientation ocular dominance columns, one for each eye; differential activation between the two ocular dominance columns could account for the sensation of depth seen in many Klüver hallucinations.) Biased flicker-induced hallucinations (see Figure 6) are a conspicuous exception to the 1- to 2-mm cortical scale, with a spatial scale similar to the scale of the biasing stimulus; this may be helpful when studying hallucinatory pattern formation using functional imaging (Billock \& Tsou, 2007).

\section{Relation of the Ermentrout-Cowan Model to Some Familiar Pattern-Forming Systems}

When first encountering self-organizing models as complex as Equation 1, there is a temptation to accept autonomous pattern for-

\footnotetext{
${ }^{3}$ Because neural density is high, the neural interactions can be handled in a mathematically continuous fashion. This is sometimes called a neural field model, in analogy to the idealized fields used in physics (Amari, 1977; Coombes, 2005). Some models (Baker \& Cowan, 2009; Bressloff, Cowan, Golubitsky, Thomas, \& Wiener, 2001; Cowan, 1985; Henke et al., 2009; Wiener, 1994) use a single equation that explicitly deals with excitatory neurons but builds in interactions equivalent to the effects of inhibitory neurons. This is mathematically elegant and computationally advantageous, but for our neural and pedagogical purposes, it is useful to employ a structure like Equation 1.
} 


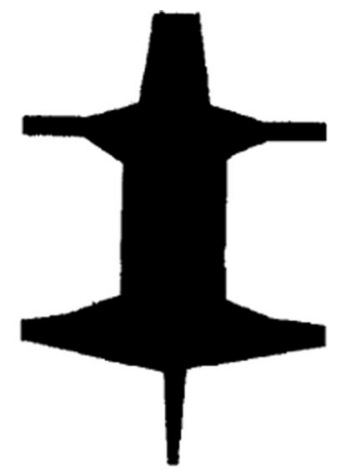

\{0i $r<0.1$

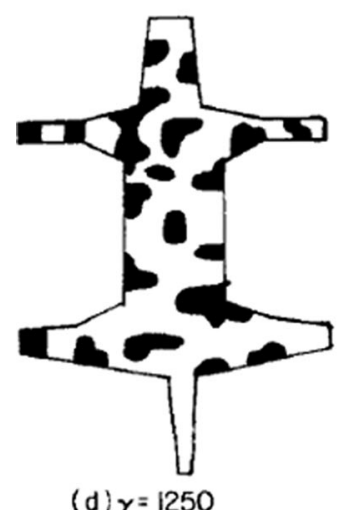

(d) $y=1250$

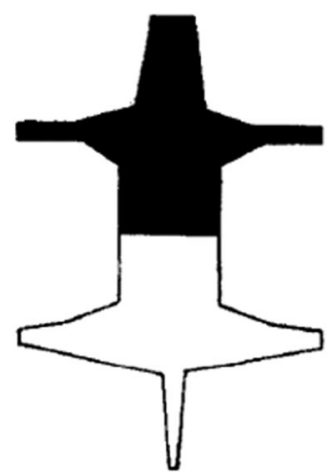

(b) $y=0.5$

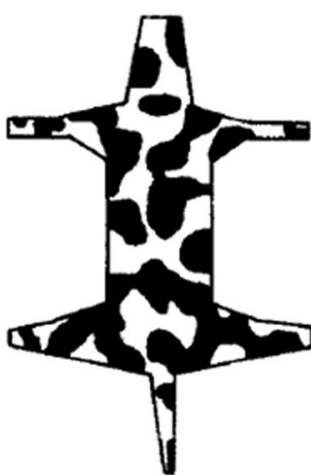

(e) $y=3000$

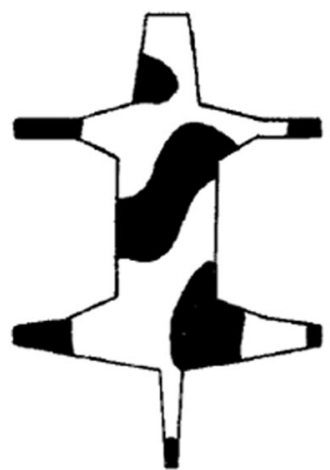

(c) $y=250$

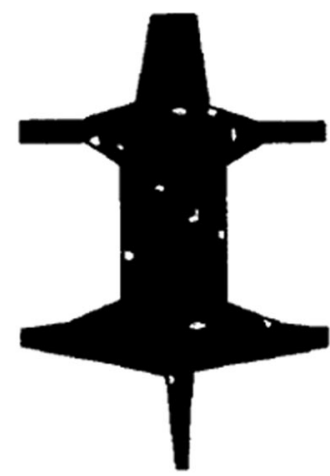

(f) $y=5000$

Figure 6. Some reaction-diffusion simulations of a spotted coat growing on an animal skin of fixed shape and variable size $(\gamma)$. Murray (1989) found that in this case, the pattern was dependent on a particular spatial scale and suggested that this is why textured coats are common in medium-sized animals but uncommon in mice and elephants. From Mathematical Biology (p. 445), by J. D. Murray, 1989, Berlin, Germany: Springer-Verlag. Copyright 1989 by Springer-Verlag. With kind permission from Springer Science+Business Media.

mation in the spirit of "... and then a miracle occurs ...," like Aphrodite sprung from the sea foam. To better understand selforganized pattern formation in neural systems, it is useful to examine other pattern-forming systems. Early work on hallucinatory modeling used fluid dynamics as an analogy: A pan of liquid, heated from below and cooled from above, can form patterns in the convecting fluid. As the bottom-heated fluid rises and the top-cooled fluid falls, currents are set up, at first at random, across the container. Reinforcing currents combine constructively, while opposing currents cancel. If a high enough temperature difference is maintained, the system can evolve into a set of mutually reinforcing rolling concentric cylinders of convecting fluid, which (if viewed from above) resemble the cortical stripes that Ermentrout and Cowan (1979) invoked to explain hallucination patterns. Moreover, three such interacting roll patterns superimposed at $60^{\circ}$ angles create a hexagonal cell pattern (often found in atmospheric phenomena; warm air rises in the center of each cell, and cold air descends on the edges) like the hexagonal textures seen in hallucinations. In simple fluid dynamics, rolls are favored when surface tension is eliminated, while hexagonal cells form if surface tension at the top of the container is substantial. In complexity theory, a variable whose change can force a sudden qualitative change in pattern formation is called a control parameter. Here, the temperature difference between top and bottom is the main control param- eter, determining if patterns can form, and surface tension is a secondary control parameter, with influence over the type of patterns formed. The constant input of heat from the bottom is dissipated to the surface by convection and requires constant renewal. Such patterns are called dissipative structures; this term has also been applied to hallucinations like those induced by flicker (Stwerka, 1993) and rather appropriately too since the hallucinations cease promptly when the outside forcing (the flicker) is removed. The effects of control parameters are also analogous in the two systems: In flicker-induced hallucinations, hexagonal forms are more often seen for weak stimulation, and fan shapes, concentric circles, and spirals (all of which stem from cortical stripe formation) are more often seen during stronger stimulation. Rule, Stoffregen, and Ermentrout's (2011) model predicts a similar effect for flicker rate. However, although fluid-dynamic pattern formation resembles hallucinatory pattern formation, mathematically there are closer systems. Here, we visit two other systemsreaction-diffusion and population dynamics - that mathematically resemble neural pattern formation to seek additional insights into the behavior of Equation 1.

Turing (1952) introduced reaction-diffusion systems. Such systems consist of two diffusing agents; one agent can activate a marker of some kind (like a skin or fur pigment), while the other agent inhibits the activation of the marker. They take the form 

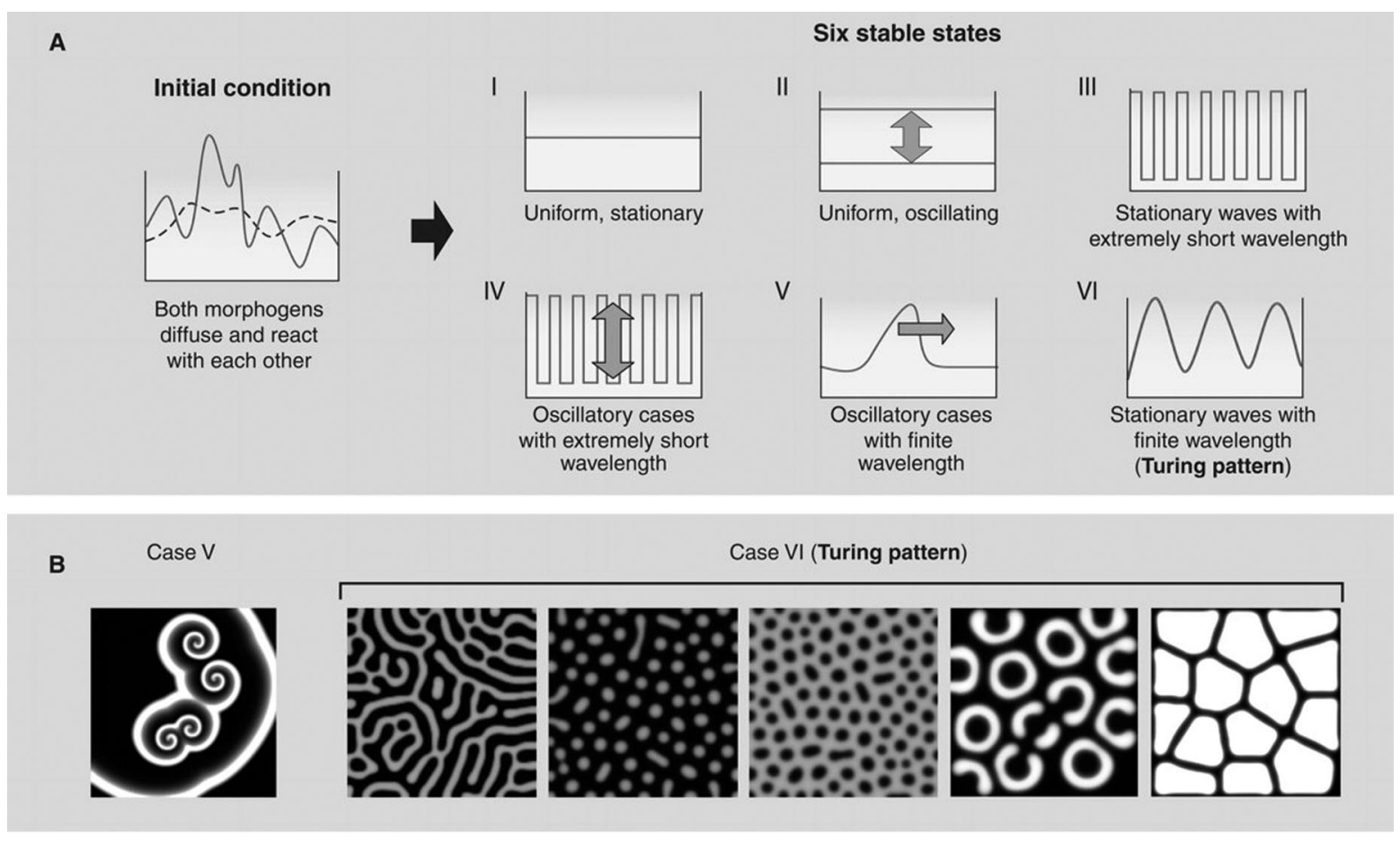

C
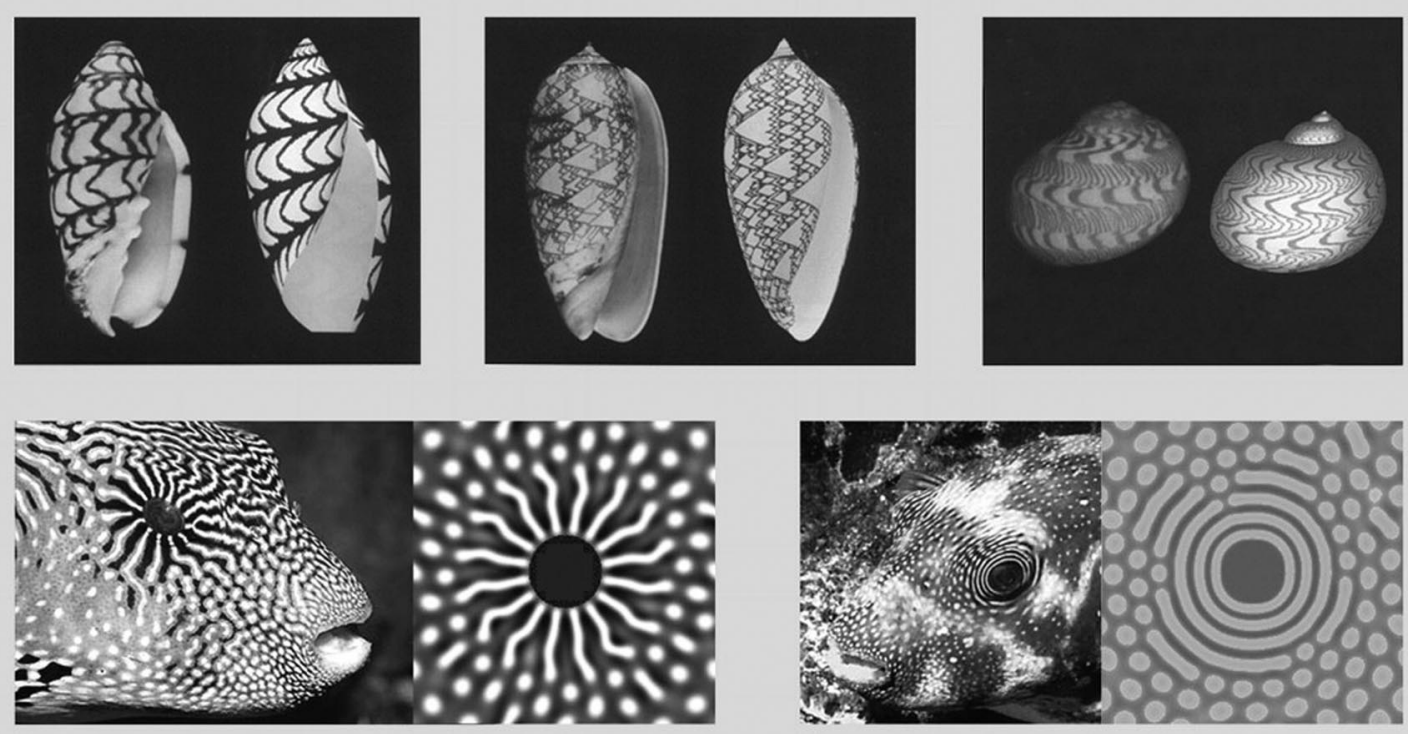

Figure 7. Reaction-diffusion (RD) simulations of pattern formation compared to actual biological patterns. A: Some stable states that RD systems can generate. B: Some two-dimensional simulations produced by a simple Turing model. C top: Actual and simulated shell patterns produced by Meinhardt's (2003) RD model. C bottom: Actual and simulated fish skin patterns produced by Sanderson, Kirby, Johnson, and Yang's (2006) RD model on fish skins. From "Reaction-Diffusion Model as a Framework for Understanding Biological Pattern Formation," by S. Kondo and T. Miura, 2010, Science, 329, p. 1618. Copyright 2010 by the American Association for the Advancement of Science. Reprinted with permission from AAAS. 

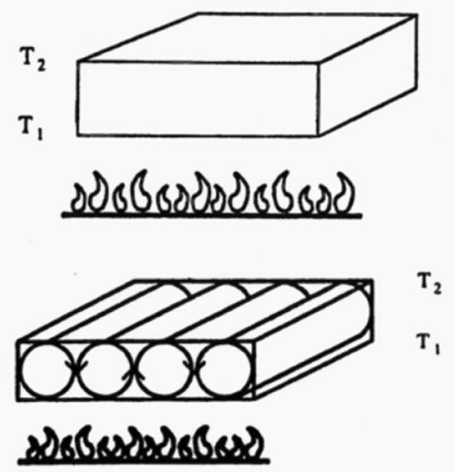

a)

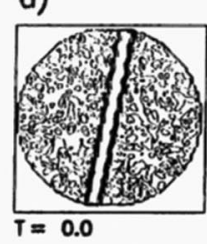

b)
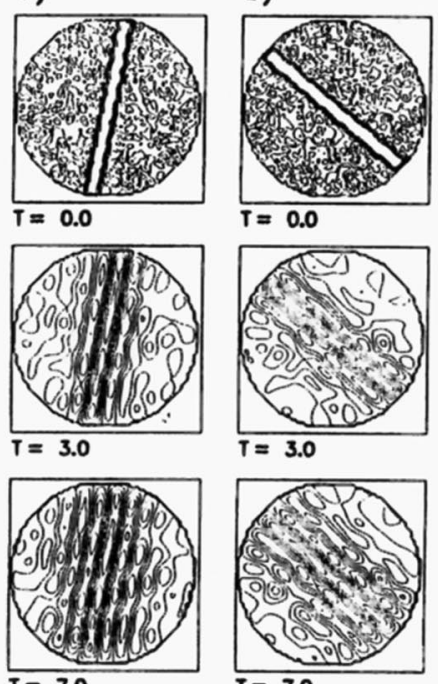

$\mathbf{T}=\mathbf{7 . 0}$
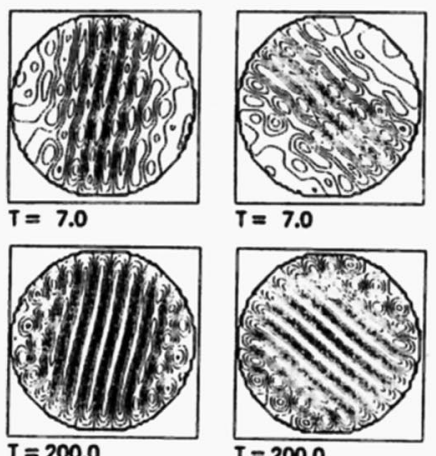

$T=7.0$

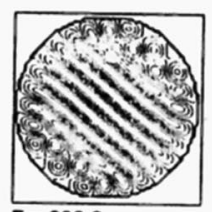

$T=\mathbf{2 0 0 . 0}$ c)
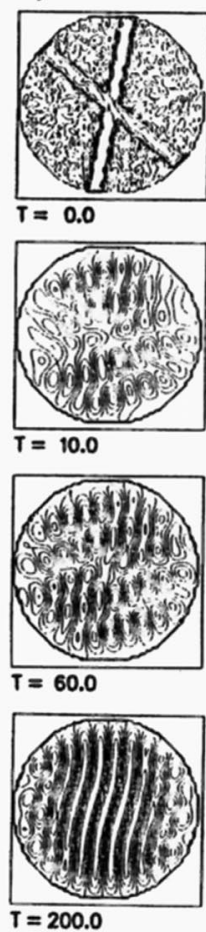

Figure 8. Biasing a classic pattern-forming system-convection patterns formed in a pan of fluid heated from below and cooled from above. Convection organizes into parallel cylinders of rising hot and falling cool fluid, which look like Ermentrout and Cowan's (1979) cortical stripes when viewed from above. The orientation of the stripes forms randomly unless biased by an outside force. The top row of the simulations shows fluid injections (white stripes) delivered at the beginning of the simulation (first studied by Bestehorn \& Haken, 1991). In Columns a and b, by 200 time steps, the biasing geometry has imposed its orientation on the entire emergent pattern. In Column c, two injections are made, one slightly stronger than the other, which compete until the stronger injection eventually dominates the entire pattern. From Principles of Brain Functioning (pp. 37 \& 241), by H. Haken, 1996, Berlin, Germany: Springer-Verlag. Copyright 1996 by Springer-Verlag. With kind permission from Springer Science+Business Media.

$$
\partial A / \partial t=\mathrm{F}(A, I)+D_{A} \nabla^{2} A ; \partial I / \partial t=\mathrm{G}(A, I)+D_{I} \nabla^{2} I,
$$

where $D_{A}$ and $D_{I}$ are diffusion rates for the activator $(A)$ and inhibitor $(I)$ chemicals, respectively, and $\nabla^{2}$ is the second derivative (Laplacian) operator $\partial^{2} x / \partial x^{2}+\partial^{2} y / \partial y^{2}$, which is used to make the diffusion flow

down the $A$ or $I$ concentration gradient. $\mathrm{F}(A, I)$ and $\mathrm{G}(A, I)$ are generally quadratic functions of chemical concentration but are specific to the system being modeled; for example, in Gierer and Meinhardt (1972),

$$
\mathrm{F}(A, I)=k_{1}-k_{2} A+k_{3} A^{2} / I ; \mathrm{G}(A, I)=k_{4} A^{2}-k_{5} I .
$$

Turing was interested in the spot-and-stripe patterns of animal coats, such as leopards and zebras, and showed that a necessary condition for the diffusion rate constants is that $D_{A} \neq D_{I}$. Because of the asymmetry between substance $A$ 's and substance $I$ 's actions ( $I$ inhibits $A$ 's action, but not vice versa), the diffusion can drive an instability. This was a revolutionary development — diffusion had previously been thought an agent of stability — but the combination of the two Turing asymmetries creates the Turing instability. Murray $(1988,1989)$ made some compelling simulations of animal coats using Turing-like reaction-diffusion models (see, e.g., Figure 6). Shoji, Iwasa, Mochizuki, and Kondo, (2002) and Kondo and Miura (2010) extended Turing models to complicated patterns found on fish skins and other surfaces (see Figure 7). The wave of cortical spreading depression-believed to be the physiological basis of migraine - is an example of a reaction-diffusion process in an excitable medium. It is therefore not surprising that several investigators have created reaction-diffusion models of the spread of the migraine fortification arc and its accompanying scotoma. Reggia and Montgomery's (1996) reaction-diffusion model acting on orientation specific neurons can result in a moving arc with irregular jagged lines that come and go, similar to the flickering serrations of fortification illusions. Dahlem and Chronicle (2004) presented an improved reaction-diffusion model that closely matches patient's migraine fortification patterns. Methods developed for studying traveling waves in reaction-diffusion systems have proven useful in studying similar waves produced by Ermentrout-Cowanlike models.

Perhaps closer to Ermentrout-Cowan models are populationbased models that resemble the simple reaction-diffusion model. As a bridge between models, Murray (1989) gifts us with the Parable of the Sweaty Grasshoppers. Imagine a host of grasshoppers in a field of dry grass. The grasshoppers have a mutation that makes them sweat excessively if they get hot. If the dry grass catches fire, nearby grasshoppers fly a distance away and start sweating so much that their surroundings become too wet to ignite when the flame-front arrives. If the sparks from the initial flame ignite many random areas, causing many random grasshopper migrations-cum-sweating-attacks, the result is a scattered pattern of burnt and unburnt areas, not unlike a Dalmatian's spotted coat. The key is that the grasshoppers must move faster than the fire $\left(D_{I}>D_{A}\right.$ in Equation 2), buying time to sweat a firebreak.

Now consider a slightly different system: The unfortunate grasshoppers avoid the fire this time but attract the attention of a group of hungry praying mantises. The interaction between the two populations is asymmetrical in several ways. The mantises inhibit the grasshoppers' numbers $G$ directly by consuming them and indirectly by reducing the number of breeders available. The grasshoppers excite the mantis population $M$ by contributing nutrients for the mantis to use for reproduction. By itself, this can lead to a temporal pattern-a boom-and-bust cycle, where the mantis numbers soar until the grasshopper numbers plummet and the mantises starve, allowing the grasshopper numbers to recover. However, there is another possibility. The grasshoppers diffuse away from the mantises' territory faster than the mantises can breed pursuers while continuing to cover their existing territory. Segel and Jackson (1972) expressed a model of a similar system (sans insect drama) as 


\begin{tabular}{|c|c|c|c|c|c|c|}
\hline $\begin{array}{c}\partial G / \partial t \\
\text { prey population growth } \\
\partial M / \partial t \\
\text { redator population growth }\end{array}$ & $\begin{array}{l}= \\
=\end{array}$ & $\begin{array}{l}a G \\
\text { births } \\
e M \\
\text { births }\end{array}$ & + & $\begin{array}{c}b G M \\
\text { violent deaths } \\
f G M \\
\text { feeding }\end{array}$ & + & $\begin{array}{c}c G^{2} \\
G \text {-cooperation } \\
g S^{2} \\
M \text {-competition }\end{array}$ \\
\hline
\end{tabular}

For some parameterizations, this kind of model leads to patchy and even periodic distributions of predator and prey (Segel \& Levin, 1976), much like the structured coloring of the Turing animal skins. Some similar models can lead to waves of pursuit and evasion (which is an interesting way to think about the advancing migraine wave front, with its leading excitatory fortifications and following inhibitory scotoma). By now, several of the connections between the Ermentrout-Cowan model and the predator-prey model should be clear, and it is not surprising that the Wilson-Cowan model (Cowan, 1968; Wilson \& Cowan, 1972, 1973) which Ermentrout and Cowan's work was based on, can be interpreted as a sophisticated kind of population dynamics model-one in which the populations become active or quiet instead of flourishing or extinct. In particular, note the terms in Equation 4 that contain the $c$ and $g$ coefficients; without these terms, the model does not produce spatial patterns. The $c$ term says that the grasshoppers cooperate with one another; the $-g$ term says that the predators fight with one another. Taken together with the other terms, this asymmetry mirrors the asymmetry of the Ermentrout and Cowan equations, where excitatory cells excite both excitatory and inhibitory cells, while inhibitory cells inhibit their fellow inhibitory cells as well as excitatory cells. Yet the careful reader should wonder, Where in the Ermentrout-Cowan model are the diffusion-like (second spatial derivative) terms that are crucial to pattern formation in both reaction-diffusion systems and population dynamics? ${ }^{4}$ The answer is that they are hidden in the very interactions of the two excitatory and two inhibitory terms in Equation 1. Because the neural excitatory and inhibitory weighting functions are Gaussian-like, with different variances, their effective interaction kernels resemble differences of Gaussians and especially second derivatives of Gaussians. These effective interaction kernels dictate the periodicity and standard scale of neural pattern formation (see Figure 9). Because a second derivative of a Gaussian is a linear operation, one can rearrange terms to isolate a naked second derivative operator, which is the Laplacian operator used in the diffusion terms of reaction-diffusion equations and predator-prey models (for the relevant math in a wonderfully different context, see Marr \& Hildreth, 1980). ${ }^{5}$

\section{Pattern Formation in the Ermentrout-Cowan Model}

If the spatial ranges of cortical excitation and inhibition differ and the level of spatially uniform excitation is raised sufficiently high, the neuronal network in Equation 1 gives rise to periodic parallel stripes of neural activity and inactivity that can form at any orientation (Ermentrout \& Cowan, 1979). The patterning is due to the local excitation-lateral inhibition connectivity built into the excitatory cells in Equation 1. The pattern begins as a small local firing rate increase but grows due to local excitation, resulting in an inhibited firing rate in the flanking regions. The inhibited flanks cannot themselves inhibit their flanks, allowing excitation to grow in the flanks of the flanks and so on. Thus, stripes of activation and inhibition form a periodic grating-like pattern whose scale is determined by the ranges of the local excitation and inhibition. There are two routes to raising the overall level of spatially uniform excitation. The first-used to model drug hallucinations and epilepsy/migraine aura-involves breaking the balance between the neural strengths of excitation and inhibition. Ermentrout and Cowan (1979) induced internal excitation by increasing $a$ and $c$ in Equation 1. However, the near symmetry of the equations suggests that a similar result could be obtained by decreasing inhibition ( $b$ and $d$ ), as some have considered for percepts induced by migraine and epilepsy (Dahlem \& Chronicle, 2004; Tass, 1995, 1997). Tass (1997) studied pattern formation for every combination of increasing or decreasing the four excitatory and inhibitory parameters in Equation 1. Some parameterizations allow only one hallucinatory state (dictated by random initial conditions) to emerge; other parameterizations allow as many as four hallucinatory states to alternate, leading to perceptual multistability. Weaker coupling between the excitatory and inhibitory elements of the network (the $b$ and $c$ terms of Equation 1) favors simple and stable geometric hallucinations, while stronger coupling leads to oscillation between competing states (e.g., perception of fan shapes alternating with perception of spirals and concentric circles; Tass, 1997).

The other route is via a spatially uniform input (the Input term in Equation 1). Because visual neurons have little response to static spatially uniform stimuli, many relatively uniform stimuli that produce hallucinatory pattern formation are temporally modulated (e.g., flicker, pulsed TMS, AC electric stimulation). This could help account for the temporal instability of many induced hallucinations-each temporal modulation is both a stimulus to pattern formation and a perturbation, disturbing the previously elicited state-but other factors likely influence the temporal scales of hallucinations. Rule et al. (2011) experimented with variations of Equation 1 (which autonomously produces produce $13 \mathrm{~Hz}$ damped oscillations). At low forcing frequencies (e.g., below $13 \mathrm{~Hz}$ ), the system responds at the forcing frequency and tends to generate stable hexagonal gratings, like those that Fechner (1838) reported on rotating black-and-white tops. At high forcing frequencies, the system breaks into two populations, each of which responds to every other cycle of the stimulus, and tends to generate stripe patterns. This subsampling (called frequency demultiplication by neural theorists) was also a feature of Drover and Ermentrout's (2006) retinal-based model of electrically induced wake contour

\footnotetext{
${ }^{4}$ Here, diffusion-like indicates spread of neural activity, not movement of substances.

5 The exact form of the spatial derivative term is probably not crucial; Cowan's (1985) model shows a fourth derivative-of-a-Gaussian-like interaction kernel for the entire system (see his Figure 12.7, p. 236), but in spatial frequency terms, this is much like a second derivative tuned to a narrower range of higher spatial frequencies.
} 


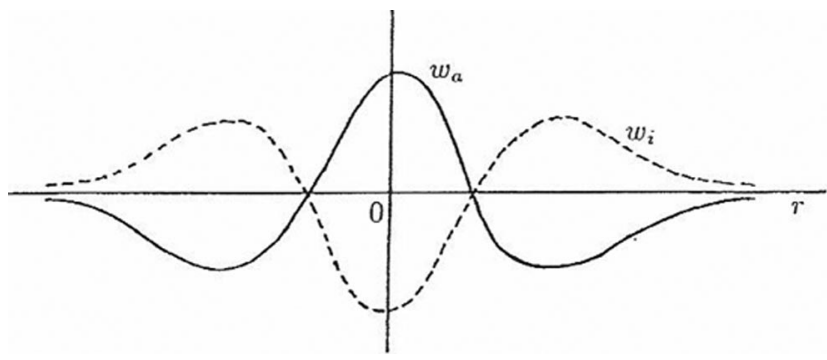

Figure 9. Second derivative-like spatial interaction kernels embedded in the Ermentrout-Cowan model (see Equation 1 in the text; Murray, 1989). These kernels are a systems-level mathematical analogue to the line-spread functions measured in single cell electrophysiology. Here, $W_{a}$ (the solid line) is the interaction built into the top equation of Equation 1; for example, it represents the $a W_{E E} * E-b W_{I E} * I$ interaction in the $E$ (excitation) growth equation. $W_{i}$ (the dashed function) is the $c W_{E I} * E-$ $d W_{I I} * I$ interaction in the $I$ (inhibition) growth equation. Thus, inhibition is the mirror image of excitation, and every stripe of excitatory activity generated by the pattern is flanked by stripes of inhibitory activity (and vice versa). The $a$ and $i$ indices that Murray (1989) used mirror the activator-inhibitor terms of reaction-diffusion theory and were used to address anatomical stripe development on striate cortex. Each three-lobed function is like a scale-specific version of the Laplacian (second derivative) terms in Equations 2 and 4 in the text. Alternately, one can think of these functions as Laplacians that have operated on Gaussians. Laplacian-ofGaussian operators are ubiquitous in vision and neuroscience, especially in the neuroscience of lateral inhibition (where they go by names like Mexican-hat functions, difference of Gaussians, and difference of offset Gaussians). From Mathematical Biology (p. 491), by J. D. Murray, 1989, Berlin, Germany: Springer-Verlag. Copyright 1989 by Springer-Verlag. With kind permission from Springer Science+Business Media.

hallucinations. Because each location in perceptual space is represented by a cell that responds on every other cycle, this creates standing wave patterns on cortex, with the two populations responding both temporally and spatially out of phase with one another. The overall effect should resemble the directionally multistable rotating fan shapes and pulsating bull's-eye patterns reported by Billock and Tsou (2007). Rule et al. found that spatial scale of flicker-induced hallucinations in their model depends on the temporal forcing frequency. An interesting implication of the modeling is that the production of stripes rather than hexagons at high temporal frequencies is guaranteed if the resting level of cortical activity is sufficiently high that the average neuron will be near an inflection point in its sigmoidal input-output function. This is the hallucinatory analogue of Werner's (2007) and Plenz and Thiagarajan's (2007) contention that the nervous system is critically poised. The result may also cast light on some other interactions between intrinsic neural rhythms and hallucinatory activity.

In addition to the standing wave phenomena described by Rule et al. (2011), there are also motion instabilities in neural field models (like Ermentrout and Cowan's) that can result in traveling waves (for reviews, see Coombes, 2005; Ermentrout \& Kleinfeld, 2001; Ermentrout \& Terman, 2010). These waves are similar to those generated by reaction-diffusion equations but travel synaptically, rather than by volume transmission. Recently, it has become possible to study these waves in slices of living surgically removed cortical tissue (e.g., Golomb \& Amitai, 1997). One key theoretical result, which fits nicely with Rule et al.'s findings, is that the speed of the traveling wave is dependent on the threshold (the lower inflection point) of the sigmoidal firing function (e.g., $S_{E}$ in Equation 1; Coombes, 2005); this has been manipulated experimentally by applying electric fields across the tissue slice (Richardson, Schiff, \& Gluckman, 2005).

The similarities between the optimal flicker rate for ErmentroutCowan simple stripe-type (see, e.g., Figure 4) hallucinations (ca. 10-20 Hz; Becker \& Elliot, 2006; Billock \& Tsou, 2007), the psychophysically matched flicker rate of migraine fortification aura (18 Hz; Crotogino et al., 2001), the roughly $10 \mathrm{~Hz}$ rate for kaleidoscopic changes of Klüver-like hallucinations sometimes experienced during migraine (Sacks, 1995b), and the content of the alpha and beta bands of EEG (8-12 and 13-20 Hz, respectively) are intriguing, yet poorly understood. Certainly, the frequency dependency and temporal scaling of hallucinations may be related to resonance between the external modulation and intrinsic neural activity (Ermentrout \& Terman, 2010; Herrmann, 2001; Rule et al., 2011). It was recognized early on that despite reducing the brightness of flicker, inducing hallucinations with flicker through closed eyes is surprisingly effective, perhaps because eye closure increases the background alpha-band EEG signal, which the flicker may interact with (V. J. Walter \& Walter, 1949). This is supported by Shevelev et al.'s (2000) finding that when flicker is synchronized to the observer's alpha rhythm, pattern formation takes 2-5 s, compared to $10-15$ s for nonsynchronized trials. Similarly, Kanai, Chaieb, Antal, Walsh, and Paulus (2008) found that phosphenes are best excited by $10-12 \mathrm{~Hz}$ current applied to cortex when the observer is in the dark and by $16-20 \mathrm{~Hz}$ current when the observer is in the light, in accord with synergistic interactions with underlying EEG cortical activity measured in darkness (alpha band) and light (beta band).

In addition to providing evidence for interactions between intrinsic neural activity and outside stimulation in hallucinatory pattern formation, such studies may shed light on discrepancies between studies in frequency dependencies of flicker-induced hallucinations. Mundy-Castle (1953) pointed out that in addition to the broad range of driving frequencies, the cortex responds with an even wider range of harmonics and subharmonics, making it difficult to identify the mechanism of action. Some subjects have larger evoked responses at the first harmonic than at the fundamental driving frequency, allowing them to respond to rather low stimulus frequencies (V. J. Walter \& Walter, 1949). Seemingly minor stimulation differences could have a strong effect by modifying the intrinsic activity. Some studies use eyelids as diffusers for a bright flickering source, while other studies use open eyes, with rotating tops, strobe-illuminated ganzfelds (empty textureless fields), or flickering monitors as sources. Wackerman, Pütz, and Allefeld (2008) pointed out that although alpha-band intrinsic activity is present in both eyes-closed and eyes-open-ganzfeld viewing, the measured content of the alpha band is different for the two cases, with the alpha-band peak shifting to higher frequencies for ganzfeld viewing (similar to the viewing conditions of Kanai et al.'s, 2008, study). Becker, Gramann, Muller, and Elliott (2009) found a similar frequency shift and an additional increase in gamma-band (ca. $40 \mathrm{~Hz}$ ) activity accompanying the development of the illusory percept. 


\section{Intriguing Synergies Between Hallucinatory Conditions}

The interplay between increasing excitation via the coupling constants (e.g., $a, c$ ) and via the Input term in Equation 1 suggests that condition pairings that drive both should interact synergistically. Five lines of evidence support this: (a) Subjects given a subhallucinatory dose of mescaline immediately perceive mescaline-quality geometric hallucinations (more intense and ornate than those normally seen in flicker) when exposed to flickering light (Smythies, 1960). Similarly, Knoll, Kuger, Höffer, and Lawder (1963) reported similar hallucinatory interactions between electrical cortical stimulation and several hallucinogenic drugs. (b) As discussed above, when flicker is synchronized to the subject's alpha EEG rhythm, pattern formation is 3 to 5 times faster than for unsynchronized trials (Shevelev et al., 2000). (c) Oster (1970) reported that LSD flashbacks (see Figures 1A-1D) are easily evoked by ocular pressure, and Klüver (1966) reported that in the early prehallucinatory stage of intoxication, ocular pressure speeds drug hallucinations. Similarly, Siegel's (1992) patient triggered LSD flashbacks (resembling Figure 1F) with flickering neon lights, but only after heavy stimulant (nicotine and caffeine) use. In keeping with this, about $83 \%$ of heavy amphetamine abusers report hallucinations as a side effect (Paulseth \& Klawans, 1985). A number of studies are consistent with a net excitatory effect of hallucinogenic drugs on the visual system (Winters, 1975). For example, LSD increases visual evoked responses at both the lateral geniculate nucleus (LGN) and cortex (Purpura, 1956a, 1956b). Interestingly, diphenylhydantoin, a drug used to treat epilepsy by dampening neural excitability (usually by deactivating some sodium channels in neural membranes), is also effective in suppressing LSD flashbacks (Thurlow \& Girvin, 1971), and subjects with LSD flashbacks show an epileptic-like increase in the coherence of EEG in the absence of stimulation (Abraham \& Duffy, 2001). (d) Migraineurs, based on many lines of evidence, appear to be chronically hyperexcitable (Aurora \& Wilkinson, 2007) and, most relevantly here, between attacks have reduced thresholds for TMSinduced phosphenes (Aurora et al., 1998). The same reduction in phosphene-induction thresholds has been documented in Ecstasy users who hallucinate (Oliveri \& Calvo, 2003). (e) Flicker triggers hallucinatory auras in some migraineurs and epileptics. Like ordinary flicker-induced and ocular-pressure-induced hallucinations, photo-induced epileptic auras are easier to obtain for two eyes than one (Hess, Harding, \& Drasdo, 1974). Moreover, in animals dosed with GABA-antagonists (which block inhibition), a migraine-like cortical wave is triggered by flicker (van Harreveld \& Stamm, 1955). Many of these interactions make sense as a synergy between excitability (internal parameters set by neural wiring and drug dosage) and the Input term (an external variable tapped by flicker, ocular pressure, and TMS) of Equation 1; both mechanisms increase excitation in a spatially homogeneous way. Consider a pedestal of internal activation to which external stimulation is added: If the combined effects of steady internal activation and transient external stimulation exceed a critical level, then pattern formation occurs. In the case of drug flashbacks, previous hallucinatory experiences may influence the long-term value of Equation 1's $a-d$ parameters via Hebbian learning.

\section{Experimental Evidence of Spontaneous Pattern Formation in Visual Cortex}

Many hallucinations are explained by models based on Ermentrout and Cowan (1979). Yet the Ermentrout-Cowan model's greater significance may be that it suggests that autonomous neural activity could be perceptually meaningful. This has become an important issue in sensory neuroscience (Arieli, Sterkin, Grinvald, \& Aertsen, 1996; Kenet, Bibitchkov, Tsodyks, Grinvald, \& Arieli, 2003; Ringach, 2003, 2009). It has long been known that even unstimulated visual cortex forms neural patterns; once thought to be mere noise, this activity is spatiotemporally structured and may interact in perceptually significant ways with spatiotemporal activity induced by images. For example, Kenet et al. (2003) used voltage-sensitive dyes to make high-resolution video recordings of neural activity in the visual cortex of anesthetized and unstimulated cats; this autonomous cortical activity is highly structured and matches cortical activity induced in the same animals by viewing oriented grating patterns (see Figure 10; Kenet et al., 2003; Ringach, 2003). These autonomously formed patterns are reminiscent of the Ermentrout-Cowan patterns; Goldberg, Rokni, and Sompolinsky (2004) treated both the Kenet et al. and Ermentrout-Cowan patterns as subsets of attractors in a dynamic phase space of neural states visited by the underlying nonlinear dynamic system, an interpretation reinforced by recent work on neural attractor dynamics (Ghosh, Rho, McIntosh, Kötter, \& Jirsa, 2008; Luczak, Bartho, \& Harris, 2009; Ringach, 2009). This description is supported by Kenet et al.'s (2003) results: The spontaneous neural activity continually and unpredictably switched between states describing different grating orientations, reminiscent of the unpredictability of the Ermentrout-Cowan model and the instability of many induced hallucinations. Of course, Kenet's patterns (if present in unanesthetized humans) are subliminal, since we do not perceive illusory oriented gratings wherever we look; Ringach (2009) suggested that sensory deprivation hallucinations (including clinical conditions like CBS) may stem from these patterns becoming visible. However, even subthreshold neural patterns could alter perception by interacting with stimulated activity.

\section{Opponent Interactions Between Hallucinations and Stimulus-Generated Activity}

Several lines of evidence suggest that cortical pattern formation interacts with stimulus-generated cortical activity to influence perception. Wilkinson (2004) proposed that the ictal blindness that follows visual epilepsy may be the result of high autonomous activity in visual cortex that never settles into an organized pattern, interfering with stimulus-driven visual activity without giving rise to percepts of its own. More prosaically, Fiser, Chiu, and Weliky (2004) showed that as cortex ages, the cortical modulation of sensory input by the underlying activity changes as well. Billock and Tsou $(2007,2010)$ attacked the problem from the other direction, using small physical stimuli to bias the otherwise random hallucinations evoked by flickering light. Previous studies of selforganized pattern formation in nonlinear dynamic systems found that pattern-forming systems could be biased by introducing a feature that was aligned in the desired orientation. For example, in Figure 8, a fluid-dynamic system forms oriented cylinders of rising 


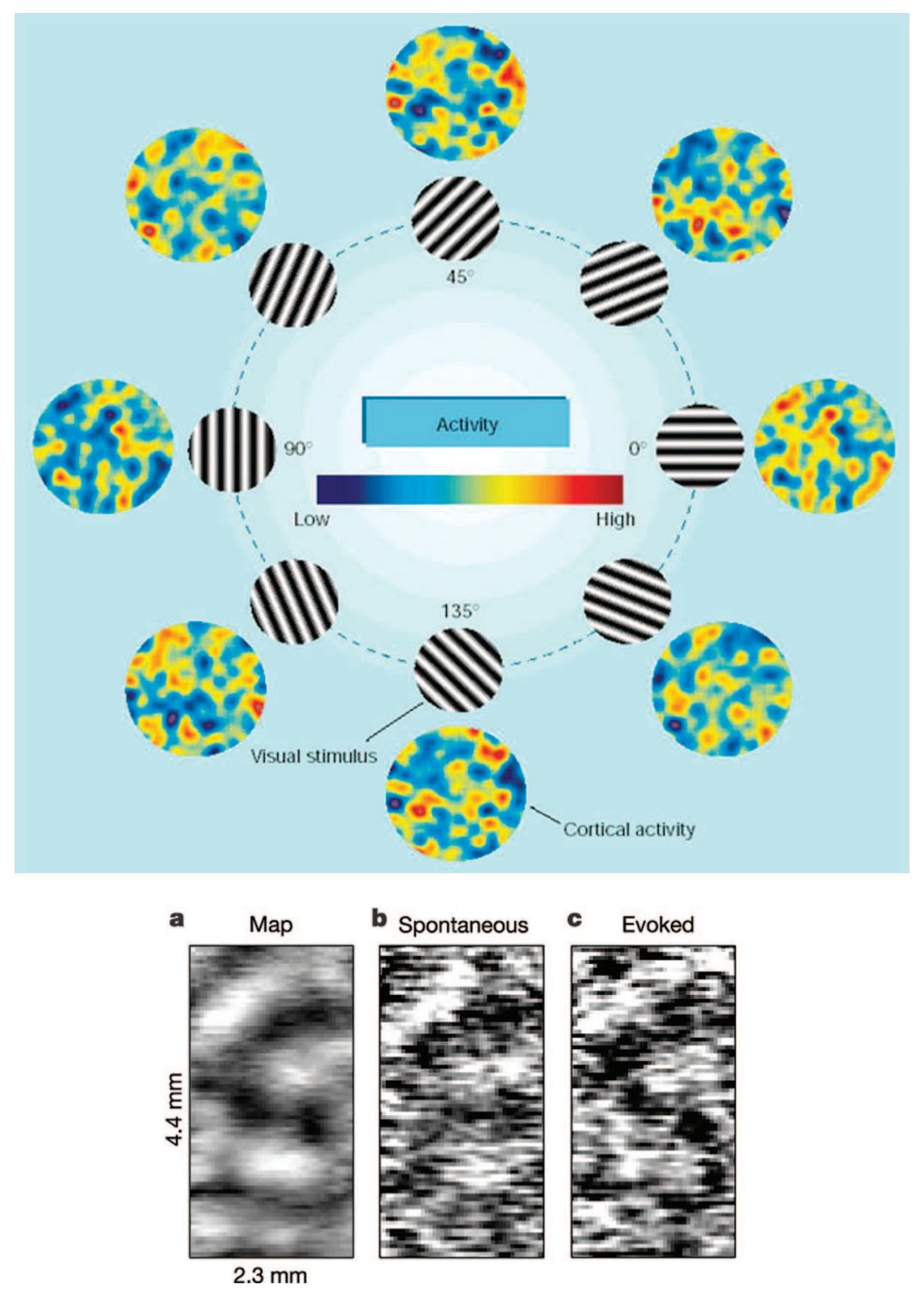

Figure 10. Spontaneous cortical pattern formation in cats. Top: Single-frame images of voltage-dependent dyes on the visual cortex evoked by oriented physical stimuli (Ringach, 2003). Bottom: Similarity of spontaneous states arising in sensory-deprived cats to the activity evoked by oriented stimuli (Kenet, Bibitchkov, Tsodyks, Grinvald, \& Arieli, 2003). a: Cortical activity evoked by presentation of vertical gratings, averaged over 160 frames. b: Single frame of spontaneous activity resembling the activity in Panel a. c: Single frame of imaged neural activity evoked by vertical grating, shown for comparison. Top panel from "Neuroscience: States of Mind," by D. L. Ringach, 2003, Nature, 425, p. 913. Reprinted by permission from Macmillan Publishers Ltd., copyright 2003. Bottom panels from "Spontaneously Emerging Cortical Representations of Visual Attributes," by T. Kenet, D. Bibitchkov, M. Tsodyks, A. Grinvald, and A. Arieli, 2003, Nature, 425, p. 954. Reprinted by permission from Macmillan Publishers Ltd., copyright 2003.

hot and falling cold fluid, which from above appear like the stripes in the Ermentrout-Cowan model. As in the Ermentrout-Cowan model, there is no preferred orientation to these stripes. However, even a single line of injected fluid can dictate the final orientation of the entire system (Bestehorn \& Haken, 1991). Billock and Tsou (2007) adapted this idea to flicker-induced hallucinations, by placing small fan-shaped or bull's-eye patterns in the midst of a uniform flickering field. They expected the hallucinatory patterns to grow from the biasing stimulus, like a geometric seed dictating crystal growth in a supersaturated solution. Instead, they found an interesting perceptual opponency between orthogonal hallucinatory geometries: Flickering around a small physical bull's-eye pattern induces a hallucinatory rotating fan shape in the flicker, whereas flickering around a small fan shape induces hallucinatory concentric circular forms that can wobble and pulsate (see Figure 11). Some patterns are more visible than others: Induced fan- 


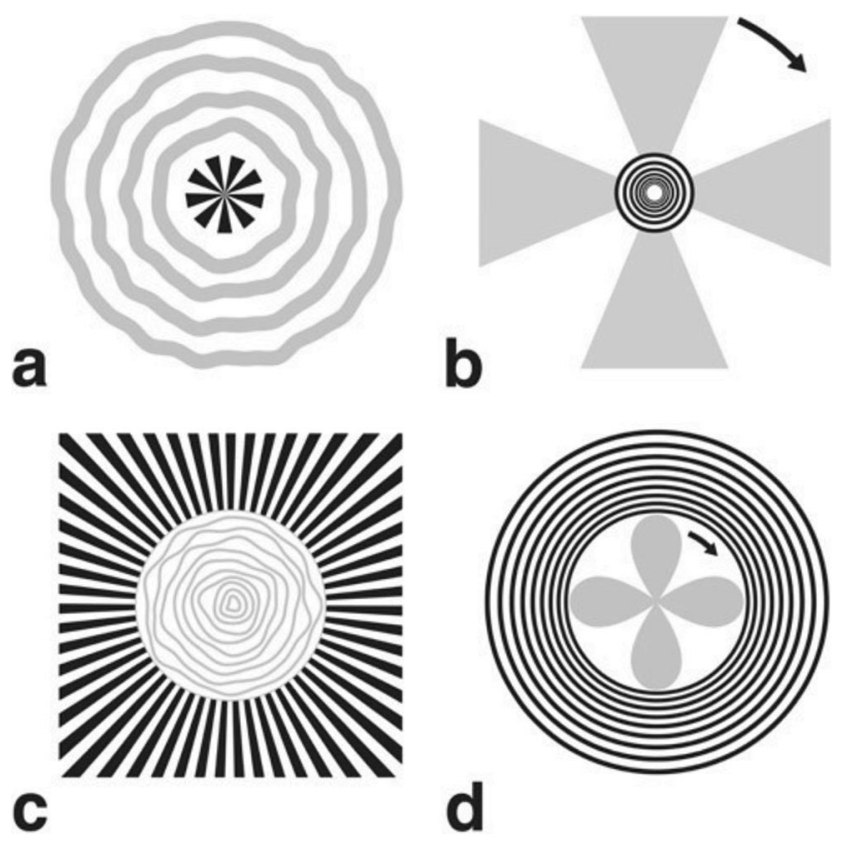

Figure 11. Biasing hallucinations. If a physical geometry (bold figures) is placed within a flickering field, the hallucination induced by the flicker (gray figures) is orthogonal to the physical stimulus; flickering around a physical set of concentric circles induces hallucinatory rotating fan shapes; flickering around physical fan shapes induces wobbling or pulsating concentric circular patterns. If the entire field flickers in phase, the hallucination extends through the physical stimulus. If the flicker does not extend through the physical form or if the form is flickered out of phase, then the hallucination is confined to the empty region beyond the physical biasing field. From "Neural Interactions Between Flicker-Induced Self-Organized Visual Hallucinations and Physical Stimuli," by V. A. Billock and B. H. Tsou, 2007, PNAS: Proceedings of the National Academy of Sciences, USA, 104, p. 8492. Copyright 2007 National Academy of Sciences, USA. Reprinted with permission.

shaped hallucinations are more salient than induced circular patterns, which in turn are more salient than induced spirals. This pattern preference resembles Kenet et al.'s (2003) finding that some spontaneous cortical patterns recur much more often than others. In general, biased hallucinations and their interactions with visual stimuli obey the same rules as perception of ordinary stimuli; these hallucinations are spatiotemporally localized phenomena, limited in space to the flickering field and rigidly locked to the presence of the physical stimulus (Billock \& Tsou, 2007).

There is evidence for temporal opponency with these same orthogonal patterns: MacKay (1957a, 1957b, 1965, 1978) saw orthogonal hallucinations (during the off phase of flickered illumination) superimposed on flickered physical geometric patterns (a sequential contrast phenomenon); as discussed earlier, he saw the same illusions as brief afterimages following prolonged viewing of a geometric pattern and in noise streaming behind transparencies of these geometries. These MacKay effect afterimage opponencies are influenced by the orientation, motion, and spatial scale of the adapting features (Georgeson, 1976, 1985). A similar opponency is seen in ambiguous textures with both circular and radial correlations; for example, after adapting to a radial geome- try, a previously ambiguous pattern of random dots (Glass patterns) appears circular (Clifford \& Weston, 2005). Two (not mutually exclusive) theories on functional interactions of these geometries have been advanced. Some researchers have noted that geometries like the fan shapes and concentric circles used by MacKay (1957a, 1957b) are the basis functions of Lie group theories of spatial vision (Caelli, 1977; Dodwell, 1991). Additionally, some masking phenomena found by Vidyasagar, Buzás, Kisvárday, and Eysel (1999) suggest that geometric opponency reduces the persistence of some visual percepts (Wede \& Francis, 2006). Since a variety of drugs and clinical conditions prolong visual persistence, it would be interesting if any of them also impact geometric opponency.

A possible fourth line of evidence for interactions between spontaneous pattern formation and physical stimuli stems from some particularly odd illusory conjunctions. Treisman (1996) treated illusory conjunctions as feature misbindings; for example, in peripheral vision, a red square and a green triangle may be misperceived as a green square and a red triangle. One experiment - on perception of forbidden colors - used vertical adjacent red/green equiluminous stripes (Billock, Gleason, \& Tsou, 2001; Billock \& Tsou, 2010). When stabilized on the eye, the border between the stripes usually collapses, and observers perceive the entire field as a novel reddish green. However, sometimes the perception of the stripes becomes multistable, and the colors appear to change sides. Although this can be understood as an ordinary illusory conjunction, Billock, Gleason, and Tsou (2001) had one subject who saw the vertical color stripes rearrange into horizontal stripes. In this particular experiment, the tops and bottoms of the vertical stripes were rounded (see Billock \& Tsou, 2004, their Figure 3), so there was no horizontal structure in the actual image for the color to misbind to. However, if humans generate Kenet-type cortical orientation patterns, odd occasional misbindings to the orientation of the Kenet patterns would be expected. If the Kenet patterns are spatially opponent for orthogonal geometries, misbindings would be expected in the orthogonal orientation to the original stimulus.

\section{Advanced Models of Hallucinatory Pattern Formation}

The spatial weighting functions ( $W_{i j}$ in Equation 1) used by Ermentrout and Cowan (1979) and Tass $(1995,1997)$ are spatially symmetric, and pattern formation arises from local reinforcement by neighboring members of the neural network; allowable patterns generated by the network include any doubly periodic pattern that tiles the visual cortex. Some neural network models allow anisotropic connectivity on cortex (Cowan, 1997; Bressloff et al., 2001, 2002; Golubitsky, Shiau, \& Török, 2003; Jirsa \& Kelso, 2000; Qubbaj \& Jirsa, 2007; Wiener, 1994), which creates new possibilities for modeling pattern perception. These networks could be useful in modeling spatial opponency between radial and concentric patterns (Billock \& Tsou, 2007). One can imagine a set of Ermentrout-Cowan-like networks, each biased to form stripes in horizontal, vertical, or oblique angles along V1, with competition between networks for the right to fire. Such a system could produce spatial opponency at a distance, if the vertical and horizontal stripe-producing networks had mutual geometrically antagonistic long-range connections preferentially arranged along particular directions on V1. Anisotropic reaction-diffusion models 
(mathematically similar to Ermentrout-Cowan neural networks) produce similar stripe-orientation opponencies; for example, in pattern formation on fish skins, if diffusion is easier in one direction than another and the inhibitor species has greater range than the activator, then stripes form parallel to the direction of diffusion until the activator's range is exceeded and then switch orientation to the perpendicular direction. Similar results occur if the activator and inhibitor species have different preferred directions of diffusion (Shoji et al., 2002). An anisotropic model should also facilitate modeling the serrated appearance of migraine fortifications and the finding that fans and concentric circles are easier to produce and more salient than spirals (Billock \& Tsou, 2007), whereas isotropic models show no such preference.

Bressloff et al.'s $(2001,2002)$ neural pattern-formation model illustrates the power of stimulus-specific networks for understanding complicated hybrid hallucinations, like textures superimposed on a geometric pattern (see, e.g., Figures $1 \mathrm{~F}$ and 12, which show a finer lattice texturing a coarser spiral structure). Cortical area V1 is functionally subdivided into an array of hypercolumns, which contain neurons with various preferences in feature orientation. In Bressloff et al.'s models, the neurons within a column interact without respect to orientation preference, but connections between cortical columns are between neurons of common orientation preference. These models behave almost as if there is a separate Ermentrout-Cowan-like pattern-forming network for each oriented population that competes for the right to fire; the patterns that arise can appear textured if the winning neurons are perceptually labeled for orientation (see also Golubitsky et al., 2003). ${ }^{6}$ The orientation of the resulting cortical stripe patterns determines the overall geometry of the illusion, while the orientation preferences of the activated neurons determine the texture attached to the geometry. Moreover, the resulting individual stripes of activity need not have the same orientation labels; richly complicated percepts, like herringbone textures, can result. Bressloff et al.'s work drastically expands the range of hallucinatory percepts that can be modeled and points the way to still more powerful treatments; one can imagine Bressloff et al.-like networks with neurons perceptually labeled for contrast, depth, color, and motion. A vast array of perceptual phenomena could be modeled by such networks. Baker and Cowan (2009) created a vector-based model that expands the number of perceptual attributes that such models can pin to spontaneous hallucinatory pattern formation. In another interesting variation, Golubitsky et al. (2003) introduced a weak level of anistrophy into the model by making connection strength vary with orientation preference (but any stimulus preference should apply). Curiously, this also creates time-varying pattern formation, with rotating and pulsating hallucinations, and tunnel-like percepts with structure seeming to emerge from or disappear into the opening of the tunnel.

\section{Stochastic Resonance in Neural/Perceptual Pattern Formation}

Stochastic resonance - a qualitative change in a system's behavior when noise is added-comes in three forms: (a) paradoxical increase in signal-to-noise ratio, (b) induced multistability, and (c) autonomous spatiotemporal pattern formation. The first two phenomena are often enhanced by using random fractal $\left(1 / \mathrm{f}^{\mathrm{n}}\right)$ noise in place of the usual white noise. However, pattern formation via
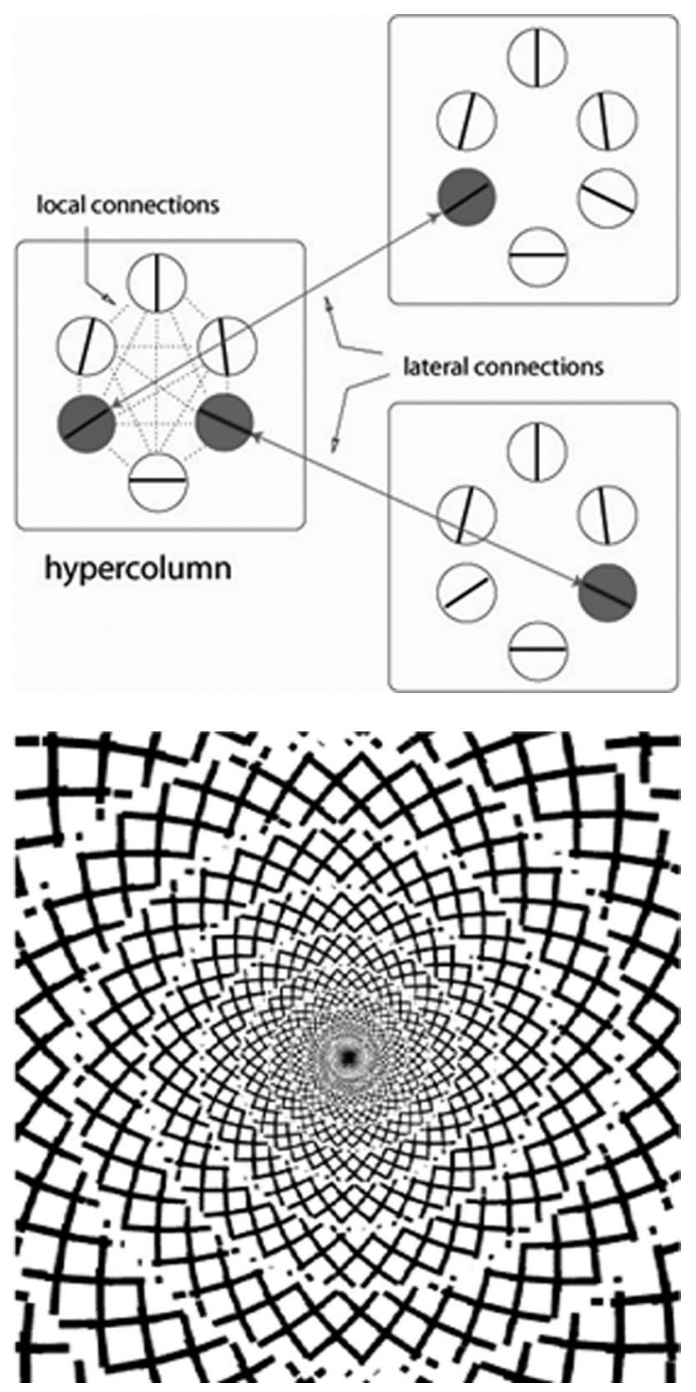

Figure 12. Neural connectivity affects perceptual pattern formation. Top: In Bressloff, Cowan, Golubitsky, Thomas, and Wiener's (2001, 2002) models, these connections can be specific to sensory neural preferences, like feature orientation. Here, local connections $(<1 \mathrm{~mm})$ within a cortical orientation hypercolumn are indiscriminate, while connections between hypercolumns are dependent on orientation selectivity. Bottom: Bressloff et al.'s model leads to textured hallucinations that resemble some complex reported hallucinations, in this case Siegel and Jarvik's (1975) THCinduced hallucination (see Figure 1F). From "Geometric Visual Hallucinations, Euclidean Symmetry and the Functional Architecture of Striate Cortex," by P. C. Bressloff, J. D. Cowan, M. Golubitsky, P. J. Thomas, \& M. C. Wiener, 2001, Philosophical Transactions of the Royal Society of London: Series B. Biological Sciences, 356, pp. 306 \& 325. Copyright 2001 by the Royal Society. Reprinted with permission.

\footnotetext{
${ }^{6}$ This explanation is an oversimplification that does not do complete justice to Bressloff et al.'s $(2001,2002)$ approach. For example, their model not only forms orientation-specific textures but also sharpens the orientation specificity of the network, relative to the specificity of the individual cortical neurons.
} 
stochastic resonance-first predicted for mathematical systems (Ditzinger, Ning, \& Hu, 1994) - is not yet well documented in neural systems; there are only a few relevant models and one related psychophysical study. For example, in a simulation of $200 \times 200$ coupled neural oscillators, 1/f noise was far more efficient than white noise in inducing spatiotemporal pattern formation (Busch, Garcia-Ojalvo, \& Kaiser, 2003; Busch \& Kaiser, 2003). Similarly, a noise input to Tass's (1995) model induces simulated concentric circles that pulsate and spirals and fans that rotate (which corresponds to scrolling of the cortical stripe patterns). These are the same dynamics that emerged in Golubitsky et al.'s (2003) model, but via a very different mechanism. Tass's model illuminates a fascinating experimental finding: Viewing dynamic fractal noise through a transparency of concentric circles generates a hallucinatory rotating fan shape, while viewing identical noise through a transparency of radial blades generates pulsating circles (Billock \& Tsou, 2007). The noise's statistics matter; spatially fractal noise requires much less contrast to generate the hallucinations than does spatially flat (white) noise. The temporal noise statistics affect the hallucinatory rotation or pulsation rate.

\section{Aspects of Perceptual Pattern Formation That Require Additional Work}

Despite these advances, gaps remain in the study of cortical and perceptual pattern formation. Many of these gaps could be filled by improved electrophysiological mapping/functional imaging techniques, but the technical problems are formidable. The more prosaic problem involves resolution of neural events. In this regard, the best spatiotemporal resolution is obtained with voltagespecific dyes on exposed primate cortex (Kenet et al., 2003)—for example, see Figure 10-but the method is unsuited for human studies. Conversely, imaging studies do map migraine trigger waves in V1 and other activity in V3a (Hadjikhani et al., 2001), but not at the resolution required to infer the percept. Biased hallucinations (see Figure 11) —which produce stable, coarse-featured patterns-may be helpful here (Billock \& Tsou, 2007). However, a more serious problem stems from the difficulties imposed by neural coding.

\section{Coding of Hallucinatory Features: Neural Substrates and Functional Imaging}

One difficulty with functional imaging is the way that the imaging signal relates to the neural activity underlying the hallucination. For example, in the Ermentrout-Cowan models that explicitly represent the excitatory and inhibitory cell populations, the stationary patterns consist of stripes of high neural activity in which excitatory activity dominates, flanked by stripes of low neural activity; such stripes-if spatiotemporally stable-should be imageable. However, for scrolling patterns, which in our experience are more common (e.g., rotating fans and spirals, pulsating circles), the inhibitory activity lags the excitatory activity, filling in the cortical grating patterns with neural activity. Inhibitory cells are at least as energetically demanding as excitatory cells, and so, their activity will blur the fMRI blood oxygen level-dependent signal (and just about any kind of imaging that is not neurotransmitter specific), limiting detection of the coarse structure of the hallucination. Even worse for imaging are Rule et al.'s (2011) standing wave patterns, where the stripes of excitation and inhibition exchange positions every $50-100 \mathrm{~ms}^{7}$ Imaging the fine structure is more difficult still; Bressloff et al.'s $(2001,2002)$ work suggests that while Ermentrout-Cowan cortical stripe formation determines the overall geometry of hallucinations, the specific neurons activated in those stripes determine what that geometry looks like: its texture, depth, contrast, color, and more. It is interesting then that many experimental conditions induce pastel or grey hallucinatory features, suggesting small asymmetries in the activation of complementary neural populations. In the early visual system, perceptually opponent mechanisms often balance one another; for example, activated off-response cells signal features darker than their background, while activation of on-response neurons should trigger perception of a bright feature. This coding of images by complementary neural populations imposes limitations on imaging those responses. Consider, for example, a lightand-dark hallucination generated by patterns of on- and offcortical cell activity, respectively; if both yield the same imageable activity on V1, then the structure of the percept would not be resolved using current functional imaging approaches. ${ }^{8}$

However, there are exceptions to the population balance rule. Brilliant colors are often reported in drug-induced hallucinations. Similarly, migraine fortifications are reported as bright, often blindingly so. Of course, this may be a reporting bias-one speaks of the bright fortification lines and not of the dark spaces between them. Indeed, some drawings of migraine fortifications depict the individual fortifications as more like grating patches than line segments (e.g., Dahlem \& Chronicle, 2004, p. 358, their Figure 5), suggesting that both on- and off-neurons can be activated during migraine, but out of phase with one another (a spatiotemporal phase shift could also account for the perceptual scintillations seen in migraine fortifications). This is essentially the same spatiotemporal phase shift considered above for scrolling of the ErmentroutCowan patterns, and it imposes the same filling-in of imageable activity problem. Similar problems will limit ability to discern hallucinatory texture, color, motion, and depth until advances in imaging overcome these limitations.

The nuances of hallucinatory perception just discussed also highlight a related problem. Although we now understand why percepts like spirals, fan shapes, and concentric circles form and can readily imagine why such forms would take on attributes like color and depth, we have relatively little understanding about differences between subjects or conditions in inducing color and depth. For example, in flicker-induced hallucinations, some observers describe the illusory colors as vivid and even unearthly, while other observers (including, alas, ourselves) see the colors as pastels. Small differences in experimental design also seem to have large effects: Most biased hallucinations are achromatic Klüver forms, although subjective colors may appear in the inducing fields (Billock \& Tsou, 2007).

\footnotetext{
${ }^{7}$ A standing wave (whose psychophysical correlate is a contrastreversing grating) is mathematically equivalent to two traveling waves, propagating through one another in opposite directions (Kelly, 1985).

${ }^{8}$ Tootell et al.'s (1998) retinocortical mapping cleverly avoided this problem by using an image (see Figure 3) whose voids were designed to elicit minimal neural response, while the image features (made of contrastreversing checks) were designed to create maximal imaging signals.
} 


\section{Other Complications: Alternate Pattern-Formation Mechanisms and Neural Loci}

Complicating this picture are alternative mechanisms that could lead to hallucinatory geometric patterns. For example, consider rotating spirals and pinwheels: In the Ermentrout-Cowan model, these correspond to a pattern of oblique stripes of neural activity on cortex (angle of orientation on cortex determines spiral tightness), scrolling perpendicularly to the orientation of the stripes. Alternately, some systems of reactive media produce spreading scroll-like waves that in two dimensions are actual spirals. Fohlmeister, Gerster, Ritz, and van Hemman (1995) modeled an Ermentrout-Cowan-like system that produces both stripe patterns and actual scrolling spiral waves on cortex, whose perceptual correlates could be hard to distinguish under some conditions. Ermentrout and Terman (2010) created a coupled neural oscillator model that produces rotating spiral waves on simulated cortex; a network of excitatory cells with time delays can behave similarly (Sacks, 1995b). Models incorporating synaptic-like time delays can also yield temporal oscillations (ca. $10 \mathrm{~Hz}$ ) that resemble the illusory flicker seen in CBS and visual epilepsy (Henke, Robinson, Drysdale, \& Loxley, 2009). Similarly, Ermentrout (1984) and Rule et al. (2011) noted that the Ermentrout and Cowan (1979) model can producing oscillations of $13-20 \mathrm{~Hz}$, similar to migraine scintillations. Another complication is that some classes of visual hallucinations may be influenced by activity in areas other than striate cortex (V1). For example, some cells in cortical area V4 are sensitive to concentric circles and fan shapes (Gallant, Connor, Rakshit, Lewis, \& Van Essen, 1996); V3a (and possibly V8) is active in some migraines (Hadjikhani et al., 2001). Significantly, ffytche (2008) found increases in activity throughout visual and frontal-parietal cortex during flicker-induced hallucinations; activity in visual cortex was centered on V4. Activity in V1 was not particularly elevated compared to nonhallucinating flicker controls, but the Ermentrout-Cowan model does not require a net increase in V1 activity, but rather an increase in spatial structuring of excitatory and inhibitory activity, which is not currently imageable (as discussed above). If other cortices are involved in autonomous spatiotemporal pattern formation, they may further complicate matters by feeding back their activity to V1. It would be extremely interesting to find out if perception of circular and fan-shaped hallucinations could be altered by deactivating V4 temporarily (e.g., by TMS) or permanently by surgery in a patient with pathological V4 lesions. An analogous experiment would monitor migraine percepts before and after V3a and V8 deactivation. It would also be desirable to monitor activity in V1 layer 6 neurons. Although flicker is a fine stimulus for the LGN, ffytsche found an interesting loss of activity in LGN during flicker-induced hallucinations, relative to flicker trials without hallucinations. Although LGN is often thought of as a relay station from retina to cortex, there are an order of magnitude more neurons contacting LGN from layer 6 of V1 than there are LGN cells projecting to V1. This massive feedback pathway is believed to selectively modulate/regulate LGN sensitivity and may be activated by patternforming activity in V1. Because the layer 6 feedback is spatially specific, it may also spatially shape the input to $\mathrm{V} 1$ patternforming mechanisms.

\section{Pattern-Formation Mechanisms in Phosphene and Fortification Percepts}

Much effort has gone into elucidating the self-organized patternformation mechanisms that underlie geometric hallucinations. We have already seen several connections between geometric hallucinations, phosphenes, and fortification percepts, especially in their spatial and temporal scales and in the ways that the same conditions can elicit combinations of the three percepts. Although not as much effort has gone into understanding the nonlinear dynamics of phosphenes and fortifications auras, it is worthwhile to examine some exceptions. Single spot-like phosphenes-most common for ocular pressure and direct cortical electric stimulation-are often best understood as focal activation of one or more neighboring neurons, within a single cortical column. They can however be created by pattern-forming mechanisms, like Sacks's (1995b) time-delay excitatory neural network, which also models Klüver patterns. Multiple phosphenes are an interesting problem. Ermentrout-Cowan models can produce regular arrays of phosphenes - the polyopia phenomena often noted for some clinical conditions and often seen for drug and flicker-induced hallucinations. Reaction-diffusion-like mechanisms-which can model migraine fortification arcs (Dahlem \& Chronicle, 2004)—would also be able to model phosphene polyopia and stripe formation in vision (which resembles the spot-and-stripe formation on animal coats that motivated reaction-diffusion theory). For example, when nonequiluminant color borders are retinally stabilized, the borders can collapse, and the colors can seem to slowly diffuse across the former boundaries, like an image made of melting wax. As the colors flow and mix, they can form patterns, like red streaks on a green background or blue glitter on a yellow background (Billock, Gleason, \& Tsou, 2001; Billock \& Tsou, 2010), much like the spotted and striped animal coats that are formed by reaction-diffusion systems (Murray, 1989; Turing, 1952). Although, at first blush, diffusion seems like an odd mechanism to invoke, filling-in mechanisms are ubiquitous in vision and often mimic a high-velocity diffusion-like process (see Paradiso \& Nakayama, 1991, who found a fast cortical spread speed of at least $150 \mathrm{~mm} / \mathrm{s}$ by using temporal masking as a probe). In this regard, the slowest color diffusion seen by Billock, Gleason, and Tsou (2001) would lie between the normal very fast filling-in process and the very slow movement of migraine activity. Of course, given the conceptual similarity of the Ermentrout-Cowan model to a reaction-diffusion system, a unified model that tackles the sequential development of phosphenes, fortifications, and Klüver geometries within a single migraine attack should be feasible. Along these lines, it is worthwhile to consider cases that blur the distinction between phosphenes and Klüver patterns.

\section{The Blurry Distinction Between Phosphenes and Klüver Forms}

As discussed earlier, phosphenes show the same kind of nonlinear scaling with position in the visual field as geometric hallucinations and migraine fortifications. When phosphenes occur in large numbers (polyopia), they are often arranged in the same geometries as Klüver patterns. Yet this does not exhaust the potential links between these phenomena, which 
sometimes appear to be more a continuum of states than a set of discrete percepts. For example, in TMS of cortex, single- and double-wedge (bow-tie/butterfly) phosphenes are common. Kammer et al. (2005) found that single wedges are seen at lower stimulus intensities and that butterfly wedges extend into both visual fields at higher intensities. These also occur in epilepsy (Babb, Halgren, Wilson, Engel, \& Crandall, 1981), but a more common form for occipital epilepsy is a doughnut-shaped phosphene. This favoritism for certain forms recalls Kenet et al.'s (2003) finding that some spontaneously formed cortical activity patterns occur more frequently than others. It is also interesting to note that these particular phosphenes would correspond to single vertical or horizontal stripes of cortical activation (a double wedge results if a horizontal stripe forms symmetrically on both occipital cortices). The length and position on cortex of these stripes would determine the size and location of the phosphene. Since isolated stripes could occur early in the pattern-formation process and could wane before seeding a full periodic pattern, this could provide a route to understanding such phosphenes. Alternately, some pattern-forming systems can form single stripes as a final state (known as a bump in neural field theory; see Kishimoto \& Amari, 1979; see also Coombes, 2005; Ermentrout \& Terman, 2010). Similarly, the small rotating pinwheels and spoked stars seen in some electrical stimulations of cortex (Dobelle \& Mladejovsky, 1974; Penfield \& Rasmussen, 1950) could be small Klüver patterns created by Ermentrout-Cowan mechanisms but could also represent a small wave front of excitation, activating in sequence orientation-specific cell populations as the wave moves through a single orientation column (Schwartz, 1980), similar to a mechanism sometimes evoked to explain migraine scintillation.

\section{Eye Movements, Edge-Sharpening Mechanisms, and Hallucination}

In ordinary vision, the visual system compensates for eye movements in various ways, perceptually stabilizing the moving world, deblurring image motion for some eye movements, and blocking visual perception during fast saccades and blinks (Volkmann, 1986). Similar phenomena can be found in visual hallucinations, but the overall picture is incomplete because these phenomena are mostly the subject of observer commentary rather than systematic study. Clearly, dream imagery is compatible with rapid eye movements, as is eidetic imagery, and neither seems to shift with eye movements (Grüsser \& Landis, 1991). However, migraine hallucinations and experimentally induced phosphenes all shift in the direction of gaze for saccadic and pursuit eye movements (Grüsser, 1986). The differences may lie in whether the cortical area in which the hallucination arises is directly involved in corollarydischarge-based eye movement compensation. Evidence for additional postprocessing exists. For example, in CBS, clinical visual loss leads to sensory deprivation hallucinations that can sometimes be abolished by making saccadic (but not pursuit) eye movements (Kölmel, 1985), especially for saccades toward the normal portion of the visual field (Vaphiades, Celesia, \& Brigell, 1996). This joins findings that saccades disrupt not only active vision but visual phosphenes (Riggs, Merton, \& Morton, 1974) and visual cognition driven by mental imagery (Brockmole, Carlson, \& Irwin, 2002). Curiously, when Charles Bonnet hallucinations originate in now blind areas of peripheral visual field, they seem sharper than is normally seen in periphery. Similarly, Richards (1971) noted that converging eye movements can make migraine hallucinations seem sharper than otherwise. In both cases, the anomalous sharpening is similar to the neural deblurring that occurs for some viewing conditions (e.g., Hammett \& Bex, 1996).

\section{Pattern Formation in Other Sensory Cortices: What Would Cortical Stripes Sound Like?}

The cytoarchitecture of visual cortex is replicated in other sensory cortices; pattern formation is likely to occur in other sensory cortices and should be experienced in characteristic ways as auditory, somatosensory, or other hallucinations. For example, an unfortunate somatosensory hallucination that can occur in migraine is formication, a sensation of insects moving on or under the skin. This might be explained as the analogue of a visual fortification arc; a scintillating fortification wave front moving through somatosensory cortex could result in a sensation of spreading irregular movement across a section of skin. Similarly, Klüver (1966) described a curious synesthesia experienced by a mescaline-dosed subject who reported not only seeing hexagonal textures (which he called fretwork) but also that he himself seemed to be made of the same pattern. Another subject could feel a moving cobweb sensation on his tongue. A third subject reported that his legs and feet became rotating spirals, identical to the rotating spiral he was seeing in his visual field, and that he could feel them as rotating spirals; "one has the sensation of somatic and optic unity" (p. 71). Several of these reports came from physicians and show useful insights but might carry little weight if not for similar reports of geometric percepts in somatosensory synesthesia without intoxication (Cytowic, 1989). Some of Klüver's reports are suggestive of sensory binding between visual and somatosensory cortex. There is precedent for this: Tactile two-point discrimination on a limb improves if the limb is viewed under magnification, even if the magnified view is uninformative, suggesting that visual magnification forces some kind of slaved magnified remapping of somatosensory cortex (Jackson, 2001; Kennett, Taylor-Clarke, \& Haggard, 2001). Moreover, entrainment of pattern formation between cortices does not require massive coupling; in simulations intended to probe the continuity of visual hallucinations between the two visual hemispheres, Rule et al. (2011) found that only a thin band of simulated connecting neurons was enough to cause identical stripe formation in the two cortices. This begs the question, If pattern formation occurs in nonvisual cortex (and given the structural similarities between sensory cortices, it is likely), would the orientation of the stripes be as perceptually meaningful as they are in visual cortex? To answer this may require that the work that illuminated visual hallucinations in visual cortex be recapitulated for the other senses and their associated cortices. Such an effort would have three main thrusts: First, like Klüver, we need to synthesize reports of nonvisual hallucinations in synesthesia, migraine, epileptic seizures, and other inducing conditions, seeking recurrent archetyped nonvisual hallucinations that are analogues of Klüver's visual form constants. Some of these may correspond to stripe patterns on specific sensory cortices, as Klüver's visual forms did. Second, 
some theoreticians use the mapping from geometric patterns on retina to stripes on V1 to argue that these mappings evolved to allow the cortex to exploit certain regularities and invariances in image processing (Caelli, 1977; Dodwell, 1991; Schwartz, 1977, 1980); if other sensory cortices exploit similar approaches, it should be possible to derive what forms would be of special significance to the information processing taking place in these sensory cortices. Third, the insights from the first two lines of research could be used to design functional imaging studies of nonvisual cortices, using nonvisual stimuli to reveal cortical stripe formation, as Tootell, Switkes, Silverman, and Hamilton (1988; Tootell et al., 1998) did with visual cortex. This then would provide feedback to the researchers engaged in the other two lines of research.

\section{Conclusions}

It is in this sense, finally, that migraine is enthralling; for it shows us, in the form of a hallucinatory display, not only an elemental activity of the cerebral cortex, but an entire self-organizing system, a universal behavior, at work. It shows us not only the secrets of neuronal organization, but the creative heart of Nature itself.

—Oliver Sacks (1995b)

The variety of hallucinatory patterns generated by selforganized cortical pattern formation is dauntingly broad, and our understanding of these phenomena is still incomplete, but that should not keep us from celebrating the remarkable multidisciplinary convergence described above. Until recently, hallucinations were treated as disparate curiosities of vision, ill-conditioned for serious study. Today, all evidence suggests that elementary hallucinations are grounded in nonlinear dynamic neuronal networks and that hallucinations provide a window into the inner working of those networks. Geometric hallucinations are linked to phosphenes and migraine fortifications by their common spatial and temporal scales, by various theoretical commonalities, by inducers that lead to various combinations of these hallucinations, and by interactions between the many conditions that induce them. Further advances are expected from developments in functional imaging and sophisticated mathematical models. These studies should illuminate ordinary visual processing as well; the interactions of geometric hallucinations with one another and with physical stimuli obey many simple rules found in ordinary stimulusdriven vision and operate on familiar spatial and temporal neural scales. Specifically, the behavior of hallucinatory percepts suggests that neural pattern formation in visual cortex is governed by the same cortical properties of localized processing, lateral inhibition, simultaneous and sequential contrast, saccadic interference, and perceptual opponency that govern ordinary vision. Hallucinatory patterns arise from ordinary cortical mechanisms driven in extraordinary ways.

\section{References}

Abraham, H. D., \& Duffy, F. H. (2001). EEG coherence in post-LSD visual hallucinations. Psychiatry Research: Neuroimaging, 107, 151-163. doi: 10.1016/S0925-4927(01)00098-1

Aleman, A., \& Larøi, F. (2008). Hallucinations: The science of idiosyncratic perception. Washington, DC: American Psychological Association. doi:10.1037/11751-000
Allefeld, C., Pütz, P., Kastner, K., \& Wackermann, J. (2011). Flicker-light induced visual phenomena: Frequency dependence and specificity of whole percepts and percept features. Consciousness and Cognition, 20, 1344-1362. doi:10.1016/j.concog.2010.10.026

Amari, S. (1977). Dynamics of pattern formation in lateral-inhibition type neural fields. Biological Cybernetics, 27, 77-87. doi:10.1007/ BF00337259

Arieli, A., Sterkin, A., Grinvald, A., \& Aertsen, A. (1996, September 27). Dynamics of ongoing activity: Explanation for the large variability in evoked cortical responses. Science, 273, 1868-1871. doi:10.1126/ science.273.5283.1868

Aurora, S. K., Ahmad, B. K., Welch, K. M., Bhardhwaj, P., \& Ramadan N. M. (1998). Transcranial magnetic stimulation confirms hyperexcitability of occipital cortex in migraine. Neurology, 50, 1111-1114. Retrieved from http://neurology.org/content/50/4/1111.full

Aurora, S. K., \& Wilkinson, F. (2007). The brain is hyperexcitable in migraine. Cephalalgia, 27, 1442-1453. doi:10.1111/j.1468-2982 2007.01502.x

Babb, T. L., Halgren, E., Wilson, C., Engel, J., \& Crandall, P. (1981). Neuronal firing patterns during the spread of an occipital lobe seizure to the temporal lobes in man. Electroencephalography and Clinical Neurophysiology, 51, 104-107. doi:10.1016/0013-4694(81)91513-3

Bak, M., Girvin, J. P., Hambrecht, F. T., Kufta, C. V., Leob, G. E., \& Schmidt, E. M. (1990). Visual sensations produced by intracortical microstimulation of the human occipital cortex. Medical and Biological Engineering and Computing, 28, 257-259. doi:10.1007/BF02442682

Baker, T. I., \& Cowan, J. D. (2009). Spontaneous pattern formation and pinning in the primary visual cortex. Journal of Physiology (Paris), 103, 52-68. doi:10.1016/j.jphysparis.2009.05.011

Becker, C., \& Elliot, M. A. (2006). Flicker-induced color and form: Interdependencies and relation to stimulation frequency and phase. Consciousness and Cognition, 15, 175-196. doi:10.1016/j.concog.2005 .05 .004

Becker, C., Gramann, K., Muller, H. J., \& Elliott, M. C. (2009). Electrophysiological correlates of flicker-induced color hallucinations. Consciousness and Cognition, 18, 266-276. doi:10.1016/j.concog2008 .05 .001

Bestehorn, M., \& Haken, H. (1991). Associative memory of a dynamical system: The example of the convection instability. Zeitschrift für Physik $B, 82,305-308$. doi:10.1007/BF01324341

Bexton, W. H., Heron, W., \& Scott, T. H. (1954). Effects of decreased variation in the sensory environment. Canadian Journal of Psychology/ Revue canadienne de psychologie, 8, 70-76. doi:10.1037/h0083596

Billock, V. A., Cunningham, D. W., Havig, P. R., \& Tsou, B. H. (2001). Perception of spatiotemporal random fractals: An extension of colorimetric methods to the study of dynamic texture. Journal of the Optical Society of America: A. Optics, Image Science \& Vision, 18, 2404-2413. doi:10.1364/JOSAA.18.002404

Billock, V. A., Gleason, G., \& Tsou, B. H. (2001). Perception of forbidden colors in retinally stabilized equiluminant images: An indication of softwired cortical color opponency? Journal of the Optical Society of America: A. Optics, Image Science \& Vision, 18, 2398-2403. doi: 10.1364/JOSAA.18.002398

Billock, V. A., \& Tsou, B. H. (2004). What do catastrophic visual binding failures look like? Trends in Neurosciences, 27, 84-89. doi:10.1016/ j.tins.2003.12.003

Billock, V. A., \& Tsou, B. H. (2006). Fechner-Benham subjective colors do not induce McCollough after-effects. Spatial Vision, 19, 161-172. doi:10.1163/156856806776923443

Billock, V. A., \& Tsou, B. H. (2007). Neural interactions between flickerinduced self-organized visual hallucinations and physical stimuli. PNAS. Proceedings of the National Academy of Sciences, USA, 104, 84908495. doi:10.1073/pnas.0610813104

Billock, V. A., \& Tsou, B. H. (2010, February). Seeing forbidden colors. 
Scientific American, 302(2), 72-77. doi:10.1038/scientificamerican 0210-72

Billock, V. A., \& Tsou, B. H. (2011). To honor Fechner and obey Stevens: Relationships between psychophysical and neural nonlinearities. Psychological Bulletin, 137, 1-18. doi:10.1037/a0021394

Bonhoeffer, T., \& Grinvald, A. (1991, October 3). Iso-orientation domains in cat visual cortex are arranged in pinwheel-like patterns. Nature, 353, 429-431. doi:10.1038/353429a0

Boroojerdi, B., Meister, I. G., Foltys, H., Sparing, R., Cohen, L. G., \& Töpper, R. (2002). Visual and motor cortex excitability: A transcranial magnetic stimulation study. Clinical Neurophysiology, 113, 1501-1504. doi:10.1016/S1388-2457(02)00198-0

Brady, J. S. (1954). The role of frequency selective reverberatory nets in cerebral function. Electroencephalography and Clinical Neurophysiology, 6, 473-478. doi:10.1016/0013-4694(54)90063-2

Bressloff, P. C., Cowan, J. D., Golubitsky, M., Thomas, P. J., \& Wiener, M. C. (2001). Geometric visual hallucinations, Euclidean symmetry and the functional architecture of striate cortex. Philosophical Transactions of the Royal Society of London: Series B. Biological Sciences, 356, 299-330. doi:10.1098/rstb.2000.0769

Bressloff, P. C., Cowan, J. D., Golubitsky, M., Thomas, P. J., \& Wiener, M. C. (2002). What geometric hallucinations tell us about the visual cortex? Neural Computation, 14, 473-491. doi:10.1162/ 089976602317250861

Brewster, D. (1834). On the influence of successive impulses of light upon the retina. London Edinburgh Philosophical Magazine Journal of Science, 4, 241-245.

Brindley, G. S. (1955). The site of electrical excitation of the human eye. Journal of Physiology, 127, 189-200. Retrieved from http:// jp.physoc.org/content/127/1/189.full.pdf

Brindley, G. S., \& Lewin, W. S. (1968). The sensations produced by electrical stimulation of the visual cortex. Journal of Physiology, 196, 479-493. Retrieved from http://jp.physoc.org/content/196/2/479.full .pdf

Brockmole, J. R., Carlson, L. A., \& Irwin, D. E. (2002). Inhibition of attended processing during saccadic eye movements. Perception \& Psychophysics, 64, 867-881. doi:10.3758/BF03196792

Brown, C. R., \& Gebhard, J. W. (1948). Visual field articulation in the absence of spatial stimulus gradients. Journal of Experimental Psychology, 38, 188-200. doi:10.1037/h0054433

Busch, H., Garcia-Ojalvo, J., \& Kaiser, F. (2003). Influence of spatiotemporal $1 / \mathrm{f}^{\alpha}-$ noise on structure formation in excitable media. Proceedings of the SPIE, 5114, 468-477. doi:10.1117/12.492943

Busch, H., \& Kaiser, F. (2003). Influence of spatiotemporally correlated noise on structure formation in excitable media. Physical Review E, 67, 041105. doi:10.1103/PhysRevE.67.041105

Caelli, T. (1977). Psychophysical interpretations and experimental evidence for the LTG/NP theory of perception. Cahiers Psychologie, 20, $107-134$

Carpenter, R. H. S. (1973). Contour-like phosphenes from electrical stimulation of the human eye: Some new observations. Journal of Physiology, 229, 767-785. Retrieved from http://ncbi.nlm.nih.gov/pmc/articles/ PMC1350562

Chadaide, Z., Arlt, S., Antal, A., Nitsche, M. A., Lang, N., \& Paulus, W. (2007). Transcranial direct current stimulation reveals inhibitory deficiency in migraine. Cephalalgia, 27, 833-839. doi:10.1111/j.14682982.2007.01337.x

Chervin, R. D., Pierce, P. A., \& Connors, B. W. (1988). Periodicity and directionality in the propagation of epileptiform discharges across neocortex. Journal of Neurophysiology, 60, 1695-1713. Retrieved from http://jn.physiology.org/content/60/5/1695.full.pdf + html

Chronicle, E. P., Wilkins, A. J., \& Coleston, D. M. (1995). Thresholds for detection of a target against a background grating suggest visual dys- function in migraine with aura but not migraine without aura. Cephalalgia, 15, 117-122. doi:10.1046/j.1468-2982.1995.015002117.x

Clifford, C. W., \& Weston, E. (2005). Aftereffect of adaptation to Glass patterns. Vision Research, 45, 1355-1363. doi:10.1016/j.visres .2004.12.016

Collerton, D., Perry, E., \& McKeith, I. (2005). Why people see things that are not there: A novel perception and attention deficit model for recurrent complex visual hallucinations. Behavioral and Brain Sciences, 28, 737-757. doi:10.1017/S0140525X05000130

Coombes, S. (2005). Waves, bumps and patterns in neural field theories. Biological Cybernetics, 93, 91-108. doi:10.1007/s00422-005-0574-y

Cowan, J. D. (1968). Statistical mechanics of nervous nets. In E. R Caianiello (Ed.), Neural networks (pp. 181-188). New York, NY: Springer.

Cowan, J. D. (1977). Some remarks on channel bandwidths for visual contrast detection. Neurosciences Research Program Bulletin, 15, 492 515

Cowan, J. D. (1985). What do drug-induced visual hallucinations tell us about the brain? In W. R. Levy, J. A. Anderson, \& S. Lehmkuhle (Eds.), Synaptic modification, neuron selectivity and nervous system organization (pp. 223-241). Hillsdale, NJ: Erlbaum.

Cowan, J. D. (1997). Neurodynamics and brain mechanisms. In M. Ito, Y Miyashita, \& E. T. Rolls (Eds.), Cognition, computation, and consciousness (pp. 205-231). Oxford, England: Oxford University Press. doi: $10.1037 / 10247-014$

Crotogino, J., Feindel, A. F., \& Wilkinson, F. (2001). Perceived scintillation rate of migraine aura. Headache, 41, 40-48. doi:10.1046/j.15264610.2001.111006040.x

Cytowic, R. E. (1989). Synesthesia: A union of the senses. New York, NY: Springer.

Dahlem, M. A., \& Chronicle, E. P. (2004). A computational perspective on migraine aura. Progress in Neurobiology, 74, 351-361. doi:10.1016/ j.pneurobio.2004.10.003

Dahlem, M. A., Engelman, R., Löwel, S., \& Müller, S. C. (2000). Does the migraine aura reflect cortical organization? European Journal of Neuroscience, 12, 767-770. doi:10.1046/j.1460-9568.2000.00995.x

Dahlem, M. A., \& Hadjikhani, N. (2009). Migraine aura: Retracting particle-like waves in weakly susceptible cortex. PLoS ONE, 4, Article e5007. doi:10.1371/journal.pone.0005007

Dahlem, M. A., \& Müller, S. C. (2003). Migraine aura dynamics after reverse retinotopic mapping of weak excitation waves in the primary visual cortex. Biological Cybernetics, 88, 419-424. doi:10.1007/ s00422-003-0405-y

Dahlem, M. A., \& Müller, S. C. (2004). Reaction-diffusion waves in neuronal tissue and the window of cortical excitability. Annalen der Physik, 13, 442-449. doi:10.1002/andp.200410087

Ditzinger, T., Ning, C. Z., \& Hu, G. (1994). Resonancelike responses of autonomous nonlinear systems to white noise. Physical Review E, 50 , 3508-3516. doi:10.1103/PhysRevE.50.3508

Dobelle, W. H., \& Mladejovsky, M. G. (1974). Phosphenes produced by electrical stimulation of human occipital cortex and their application to the development of a prosthesis for the blind. Journal of Physiology, 243, 553-576. Retrieved from http://jp.physoc.org/content/243/2/ 553.full.pdf + html

Dobelle, W. H., Mladejovsky, M. G., Evans, J. R., Roberts, T. S., \& Girvin, J. P. (1976, January 15). "Braille" reading by a blind volunteer by visua cortex stimulation. Nature, 259, 111-112. doi:10.1038/259111a0

Dobelle, W. H., Quest, D. O., Autunes, J. L., Roberts, T. S., \& Girvin, J. P. (1979). Artificial vision for the blind by electrical stimulation of the visual cortex. Neurosurgery, 5, 521-527. doi:10.1227/00006123197910000-00022

Dodwell, P. C. (1991). Transformations after Gibson: Biological and ecological constraints on vision. In R. J. Watt (Ed.), Pattern perception by man and machine (pp. 19-29). Boca Raton, FL: CRC Press. 
Drover, J. D., \& Ermentrout, G. B. (2006). Phase boundaries as electrically induced phosphenes. SIAM Journal of Applied Dynamic Systems, 5, 529-551. doi: $10.1137 / 050646469$

Eichmeier, J., \& Niedermaier, S. (1976). Excitation of subjective light patterns (phosphenes) at different altitudes. International Journal of Biometeorology, 20, 304-308. doi:10.1007/BF01553587 doi:10.1007/ BF01553587

Ermentrout, B. (1984). Mathematical models for the patterns of premigrainous auras. In A. V. Holden \& W. Winlow (Eds.), The neurobiology of pain (pp. 267-283). Manchester, England: Manchester University Press.

Ermentrout, B., Campbell, J., \& Oster, G. (1986). A model for shell patterns based on neural activity. Veliger, 28, 369-388. Retrieved from http://www.math.pitt.edu/ bard/pubs/Shells.pdf

Ermentrout, G. B., \& Cowan, J. D. (1979). A mathematical theory of visual hallucination patterns. Biological Cybernetics, 34, 137-150. doi: 10.1007/BF00336965

Ermentrout, G. B., \& Kleinfeld, D. (2001). Traveling electrical waves in cortex: Insights from phase dynamics and speculation on a computational role. Neuron, 29, 33-44. doi:10.1016/S0896-6273(01)00178-7

Ermentrout, G. B., \& Terman, D. H. (2010). Mathematical foundations of neuroscience. New York, NY: Springer.

Evans, J. R., Gordon, J., Abramov, I., Mladejovsky, M. G., \& Dobelle, W. H. (1979). Brightness of phosphenes elicited by electrical stimulation of human visual cortex. Sensory Processes, 3, 82-94. Retrieved from http://ncbi.nlm.nih.gov/pubmed/515743

Fechner, G. T. (1838). Über eine Scheibe zur Erzeungung subjectiver Farben [Via a disk for generating subjective colors]. Poggendorff's Annalen der Physik und Chemie, 45, 227-232.

ffytche, D. H. (2005). Visual hallucinations and the Charles Bonnet syndrome. Current Psychiatry Reports, 7, 168-179. doi:10.1007/s11920005-0050-3

ffytche, D. H. (2008). The hodology of hallucinations. Cortex, 44, 10671083. doi:10.1016/j.cortex.2008.04.005

ffytche, D. H., Howard, R. J., Brammer, M. J., Woodruff, D. P., \& Williams, S. (1998). The anatomy of conscious vision: An fMRI study of visual hallucinations. Nature Neuroscience, 1, 738-742. doi:10.1038/ 3738

Fierro, B., Ricci, R., Piazza, A., Scalia, S., Giglia, G., Vitello, G., \& Brighina, F. (2003). $1 \mathrm{~Hz}$ rTMS enhances extrastriate cortex activity in migraine: Evidence of a reduced inhibition. Neurology, 61, 1446-1448. doi:10.1046/j.1460-9568.2003.02679.x

Fiorentini, A., \& MacKay, D. M. (1965). Temporal factors in pattern vision. Quarterly Journal of Experimental Psychology, 17, 282-291. doi:10.1080/17470216508416446

Fiser, J., Chiu, C., \& Weliky, M. (2004, September 30). Small modulation of ongoing cortical dynamics by sensory input during natural vision. Nature, 431, 573-578. doi:10.1038/nature02907

Fohlmeister, C., Gerster, W., Ritz, R., \& van Hemman, J. L. (1995). Spontaneous excitations in the visual cortex: Stripes, spirals, rings and collective bursts. Neural Computation, 7, 905-914. doi:10.1162/ neco.1995.7.5.905

Freedman, S. J., \& Marks, P. A. (1965). Visual imagery produced by rhythmic photic stimulation: Personality correlates and phenomenology. British Journal of Psychology, 56, 95-112. Retrieved from http:// www.ncbi.nlm.nih.gov/pubmed/14292059

Fried, I., Spenser, D. D., \& Spenser, S. S. (1995). The anatomy of epileptic auras: Focal pathology and surgical outcome. Journal of Neurosurgery, 83, 60-66. doi:10.3171/jns.1995.83.1.0060

Gallant, J. L., Connor, C. E., Rakshit, S., Lewis, J. W., \& Van Essen, D. C. (1996). Neural responses to polar, hyperbolic, and Cartesian gratings in area V4 of the macaque monkey. Journal of Neurophysiology, 76, 2718-2739. Retrieved from http://jn.physiology.org/content/76/4/ 2718.long
Georgeson, M. A. (1976). Psychophysical hallucinations of orientation and spatial frequency. Perception, 5, 99-111. doi:10.1068/p050099

Georgeson, M. A. (1985). Inferring cortical organization from subjective visual patterns. In D. Rose \& V. G. Dobson (Eds.), Models of the visual cortex (pp. 223-232). New York, NY: Wiley.

Ghosh, A., Rho, Y., McIntosh, A. R., Kötter, R., \& Jirsa, V. K. (2008) Noise during rest enables the exploration of the brain's dynamic repertoire. PLOS Computational Biology, 4, Article e1000196. Retrieved from http://ploscompbiol.org/article/info\%3Adoi\%2F10.1371\% 2Fjournal.pcbi.1000196

Gierer, A., \& Meinhardt, H. (1972). A theory of biological pattern formation. Kybernetik, 12, 30-39. doi:10.1007/BF00289234

Goldberg, J. A., Rokni, U., \& Sompolinsky, H. (2004). Patterns of ongoing activity and the functional architecture of the primary visual cortex. Neuron, 42, 489-500. doi:10.1016/S0896-6273(04)00197-7

Golomb, D., \& Amitai, Y. (1997). Propagating neuronal discharges in neocortical slices: Computational and experimental study. Journal of Physiology, 78, 1199-1211. Retrieved from http://jn.physiology.org/ content/78/3/1199.ful

Golubitsky, M., Shiau, L. J., \& Török, A. (2003). Bifurcation on the visual cortex with weakly anisotropic lateral coupling. SIAM Journal of Applied Dynamical Systems, 2, 97-143. doi:10.1137/S1111111102409882

Grafstein, B. (1963). Neural release of potassium during spreading depression. In M. A. B. Brazier (Ed.), Brain function: Cortical excitability and steady potentials (pp. 87-124). Berkeley: University of California Press.

Granziera, C., DaSilva, A. F. M., Snyder, J., Tuch, D. S., \& Hadjikhani, N. (2006). Anatomical alterations of the visual motion processing network in migraine with or without aura. PLOS Medicine, 3, 1915-1921. doi 10.1371/journal.pmed.0030402

Grüsser, O. J. (1986). Interaction of efferent and afferent signals in visual perception. Acta Psychologica, 63, 3-21. doi:10.1016/00016918(86)90039-9

Grüsser, O. J. (1995). Migraine phosphenes and the retino-cortical magnification faction. Vision Research, 35, 1125-1134. doi:10.1016/00426989(94)00187-Q

Grüsser, O. J., Grüsser-Cornhels, U., Kusel, R., \& Przybyzewski, A. W. (1989). Responses of retinal ganglion cells to eyeball deformation: A neurophysiological basis for "pressure phosphenes." Vision Research, 29, 181-194. doi:10.1016/0042-6989(89)90123-5

Grüsser, O. J., \& Hagner, M. (1990). On the history of deformation phosphenes and the idea of internal light generated in the eye for the purpose of vision. Documenta Ophthalmologica, 74, 57-85. doi: 10.1007/BF00165665

Grüsser, O. J., Hagner, M., \& Przybyswewski, A. (1989). The effect of dark adaptation on the response of cat retinal ganglion cells to eyeball deformation. Vision Research, 29, 1059-1068. doi:10.1016/00426989(89)90053-9

Grüsser, O. J., \& Landis, T. (1991). Visual agnosias and other disturbances of visual perception and cognition. Boca Raton, FL: CRC Press.

Hadjikhani, N. Sanchez del Rio, M., Wu, O., Schwartz, D. Bakker, D., Fischl, B., .. Moskowitz, M. A. (2001). Mechanisms of migraine aura revealed by functional MRI in human visual cortex. PNAS: Proceedings of the National Academy of Sciences, USA, 98, 4687-4692. doi:10.1073/ pnas.071582498

Haken, H. (1996). Principles of brain functioning. Berlin, Germany: Springer-Verlag.

Hammett, S. T., \& Bex, P. J. (1996). Motion sharpening: Evidence for addition of high spatial frequencies to the effective neural image. Vision Research, 36, 2729-2733. doi:10.1016/0042-6989(96)00009-0

Harding, A. J., Broe, G. A., \& Halliday, G. M. (2002). Visual hallucinations in Lewy body disease relate to Lewy bodies in the temporal lobe. Brain, 125, 391-403. doi:10.1093/brain/awf033

Henke, H., Robinson, P. A., Drysdale, P. M., \& Loxley, P. N. (2009). Spatiotemporal dynamics of pattern formation in the primary visual 
cortex and hallucinations. Biological Cybernetics, 101, 3-18. doi: 10.1007/s00422-009-0315-8

Heron, W. (with D. O. Hebb). (1961). Cognitive and physiological effects of perceptual isolation. In P. Solomon (Ed.), Sensory deprivation: A symposium held at Harvard Medical School (pp. 6-33). Cambridge, MA: Harvard University Press.

Heron, W., Doane, B. K., \& Scott, T. H. (1956). Visual disturbances after prolonged perceptual isolation. Canadian Journal of Psychology/Revue canadienne de psychologie, 10, 13-18. doi:10.1037/h0083650

Herrmann, C. S. (2001). Human EEG responses to 1-100 Hz flicker: Resonance phenomena in human visual cortex and their potential correlation to cognitive phenomena. Experimental Brain Research, 137, 346-353. doi:10.1007/s002210100682

Herschel, J. F. W. (1867). Familiar lectures on scientific subjects. London, England: Alexander Strahan.

Hess, R. F., Harding, G. F., \& Drasdo, N. (1974). Seizures induced by flickering light. American Journal of Optometry and Physiological Optics, 51, 517-529. doi:10.1097/00006324-197408000-00001

Holcombe, A. O., Macknik, S. L., Intrilgator, J., Seiffert, A. E., \& Tse, P. U. (1999). Waves and spokes: New motion-induced brightness illusions. Perception, 28, 1231-1242. doi:10.1068/p2965

Horowitz, M. J. (1978). Image formation and cognition (2nd ed.). New York, NY: Appleton.

Horton, J. C. (1996). Patterns of ocular dominance columns in human striate cortex in strabismic amblyopia. Visual Neuroscience, 13, 787795. doi: $10.1017 /$ S0952523800008658

Horton, J. C., \& Hedley-White, E. T. (1984). Cytochrome oxidase studies of human visual cortex. Philosophical Transactions of the Royal Society of London: Series B. Biological Sciences, 304, 255-272. doi:10.1098/ rstb.1984.0022

Hughlings Jackson, J. (1958). Selected writings of John Hughlings Jackson (Vols. 1-2). New York, NY: Basic Books.

Jackson, S. R. (2001). Action binding: Dynamic interactions between vision and touch. Trends in Cognitive Science, 5, 505-506. doi:10.1016/ S1364-6613(00)01792-7

James, W. (1920). The letters of William James (Vol. 2). Boston, MA: Atlantic Monthly Press.

Jirsa, V. K., \& Kelso, J. A. S. (2000). Spatiotemporal pattern formation in neural systems with heterogeneous connection topologies. Physical Review E, 62, 8462-8465. doi:10.1103/PhysRevE.62.8462

Kammer, K., Puls, K., Erb, M., \& Grodd, W. (2005). Transcranial magnetic stimulation in the visual system: II. Characterization of induced phosphenes and scotomas. Experimental Brain Research, 160, 129-140. doi:10.1007/s00221-004-1992-0

Kanai, R., Chaieb, L., Antal, A., Walsh, V., \& Paulus, W. (2008). Frequency dependent electrical stimulation of the visual cortex. Current Biology, 18, 1839-1843. doi:10.1016/j.cub.2008.10.027

Kellerman, J., Rigler, D., \& Siegel, S. (1977). The psychological effect of isolation in protected environments. American Journal of Psychiatry, 134, 563-565.

Kelly, D. H. (1985). Visual processing of moving stimuli. Journal of the Optical Society of America: A. Optics, Image Science \& Vision, 2, 216-225. doi:10.1364/JOSAA.2.000216

Kenet, T., Bibitchkov, D., Tsodyks, M., Grinvald, A., \& Arieli, A. (2003, October 30). Spontaneously emerging cortical representations of visual attributes. Nature, 425, 954-956. doi:10.1038/nature02078

Kennett, S., Taylor-Clarke, M., \& Haggard, P. (2001). Noninformative vision improves spatial resolution. Current Biology, 11, 1188-1191. doi:10.1016/S0960-9822(01)00327-X

King-Smith, P. E., Loffing, D. H., \& Jones, R. (1986). Rod and cone ERGs and their oscillatory potentials. Investigative Ophthalmology and Visual Science, 27, 270-273. Retrieved from http://iovs.org/content/27/2/ 270.full.pdf + html

Kishimoto, K., \& Amari, S. (1979). Existence and stability of local exci- tations in homogeneous neural fields. Journal of Mathematical Biology, 7, 303-318.

Klüver, H. (1966). Mescal and mechanisms of hallucinations. Chicago, IL University of Chicago Press.

Knoll, M., Kugler, J., Eichmeier, J., \& Höffer, O. (1962). Note on the spectroscopy of subjective light patterns. Journal of Analytical Psychology, 7, 55-69. doi:10.1111/j.1465-5922.1962.00055.x

Knoll, M., Kugler, J., Höffer, O., \& Lawder, S. D. (1963). Effects of chemical stimulation of electrically-induced phosphenes on their bandwidth, shape, number and intensity. Confinia Neurologica, 23, 201-226. doi: $10.1159 / 000104299$

Kölmel, H. (1985). Complex visual hallucinations in hemianopic fields. Journal of Neurology, Neurosurgery and Psychiatry, 48, 29-38. doi: 10.1136/jnnp.48.1.29

Kondo, S., \& Miura, T. (2010, September 24). Reaction-diffusion model as a framework for understanding biological pattern formation. Science, 329, 1616-1620. doi:10.1126/science.1179047

Lashley, K. S. (1941). Patterns of cerebral integration indicated by the scotomas of migraine. Archives of Neurology and Psychiatry, 46, 331339. Retrieved from http://archneurpsyc.ama-assn.org/cgi/reprint/46/2/ 331

Leão, A. A. P. (1963). On the spread of spreading depression. In M. A. B. Brazier (Ed.), Brain function: Cortical excitability and steady potentials (pp. 73-85). Berkeley: University of California Press.

Lepore, F. (1990). Spontaneous visual phenomena with visual loss: 104 patients with lesions of retinal and neuronal afferent pathways. Neurology, 40, 441-447. Retrieved from http://neurology.org/content/40/ 3_Part_1/444.full.pdf + html

Luczak, A., Bartho, P., \& Harris, K. D. (2009). Spontaneous events outline the realm of possible sensory responses in neocortical populations. Neuron, 62, 413-425. doi:10.1016/j.neuron.2009.03.014

MacKay, D. M. (1957a, October 26). Moving visual images produced by regular stationary patterns. Nature, 180, 849-850. doi:10.1038/ $180849 \mathrm{a} 0$

MacKay, D. M. (1957b, November 23). Some further visual phenomena associated with regular pattern stimulation. Nature, 180, 1145-1146. doi:10.1038/1801145c0

MacKay, D. M. (1965). Visual noise as a tool of research. Journal of General Psychology, 72, 181-197. doi:10.1080/00221309.1965 .9710688

MacKay, D. M. (1978). The time-course of induction of complementary images. Vision Research, 18, 913-916. doi:10.1016/0042 6989(78)90017-2

Marg, E., \& Rudiak, D. (1994). Phosphenes induced by magnetic stimulation over the occipital brain: Description and probable site of stimulation. Optometry and Vision Science, 71, 301-311. doi:10.1097/ 00006324-199405000-00001

Marr, D. H., \& Hildreth, E. (1980). Theory of edge detection. Proceedings of the Royal Society of London B, 207, 187-217. doi:10.1098/ rspb.1980.0020

Mayzner, M. S. (1975). Studies of visual information processing in man. In R. L. Solso (Ed.), Information processing and cognition: The Loyola Symposium (pp. 31-54). Hillsdale, NJ: Erlbaum.

McDougall, W. (1904). The variation of intensity of visual sensation with the duration of the stimulus. British Journal of Psychology, 1, 151-189. Retrieved from http://pao.chadwyck.co.uk/PDF/1298216434325.pdf

Meinhardt, M. (2003). The algorithmic beauty of seashells. Berlin, Germany: Springer-Verlag.

Merton, P. A., \& Morton, H. B. (1980, May 22). Stimulation of the cerebral cortex in the intact human subject. Nature, 285, 227. doi:10.1038/ $285227 \mathrm{a} 0$

Motokawa, K. (1970). Physiology of color and pattern vision. Berlin, Germany: Springer-Verlag.

Mulleners, W. M., Aurora, S. K., Chronicle, E. P., Stwert, R., Gopal, S., \& 
Koehler, P. J. (2001). Self-reported photophobic symptoms in migraineurs and controls are reliable and predict diagnostic category accurately. Headache, 41, 31-39. doi:10.1046/j.1526-4610.2001 $.111006031 . x$

Mulleners, W. M., Chronicle, E. P., Palmer, J. E., Koehler, P. J., \& Vredeveld, J. W. (2001). Visual cortex excitability in migraine with and without aura. Headache, 41, 565-572. doi:10.1046/j.15264610.2001.041006565.x

Mulleners, W. M., Chronicle, E. P., Vredeveld, J. W., \& Koehler, P. J. (2002). Visual cortex excitability in migraine before and after valproate prophylaxis: A pilot study using TMS. European Journal of Neurology, 9, 35-40. doi:10.1046/j.1468-1331.2002.00334.x

Mundy-Castle, A. C. (1953). Electrical responses of the brain in relation to behavior. British Journal of Psychology, 44, 318-329. Retrieved from http://ncbi.nlm.nih.gov/pubmed/13106271

Murray, J. D. (1988, March). How the leopard gets its spots. Scientific American, 258(3), 80-87. doi:10.1038/scientificamerican0388-80

Murray, J. D. (1989). Mathematical biology. Berlin, Germany: SpringerVerlag.

Oliveri, M., \& Calvo, G. (2003). Increased visual cortical excitability in ecstasy users: A transcranial magnetic stimulation study. Journal of Neurology, Neurosurgery, and Psychiatry, 74, 1136-1138. doi:10.1136/ jnnp.74.8.1136

Oster, G. (1970, February). Phosphenes. Scientific American, 222(2), 8287. doi:10.1038/scientificamerican0270-82

Palmer, J. E., Chronicle, E. P., Rolan, P., \& Mulleners, W. P. (2000). Cortical hyperexcitability is cortical under-inhibition: Evidence from a novel functional test of migraine patients. Cephalalgia, 20, 525-532. doi:10.1046/j.1468-2982.2000.00075.x

Panayiotopoulos, C. P. (1999). Elementary visual hallucinations, blindness and headache in idiopathic occipital epilepsy: Differentiation from migraine. Journal of Neurology, Neurosurgery and Psychiatry, 66, 536540. doi:10.1136/jnnp.66.4.536

Paradiso, M. A., \& Nakayama, K. (1991). Brightness perception and filling-in. Vision Research, 31, 1221-1236. doi:10.1016/0042-6989 (91)90047-9

Paulseth, J. E., \& Klawans, H. L. (1985). Drug-induced behavioral disorders. In P. J. Vinken, G. W. Bruyn, \& H. L. Klawans (Eds.), Handbook of clinical neurology: Vol. 2. Neurobehavioral disorders (pp. 591-608). Amsterdam, the Netherlands: Elsevier.

Paulus, W. (2010). On the difficulties of separating retinal from cortical origins of phosphenes when using transcranial alternating current stimulation (tACS). Clinical Neurophysiology, 121, 987-991. doi:10.1016/ j.clinph.2010.01.029

Penfield, W., \& Kristiansen, K. (1951). Epileptic seizure patterns. Springfield, IL: Charles C Thomas.

Penfield, W., \& Rasmussen, T. (1950). The cerebral cortex of man. New York, NY: Macmillan.

Plenz, D., \& Thiagarajan, T. (2007). The organizing principal of neuronal avalanches: Cell assemblies in the cortex. Trends in Neurosciences, 30 , 101-110. doi:10.1016/j.tins.2007.01.005

Purkinje, J. E. (1819/1823). Beobachtungen und versuche zur physiolgie der sinne: Beiträge zur kenntniss des schens in subjectiver hinsicht [Observations and experiments on the physiology of the senses: Contributions to the knowledge of vision in its subjective aspect]. Prague, Czechoslovakia: Calve.

Purpura, D. P. (1956a). Electrophysiological analysis of psychotogenic drug action: I. Effect of LSD on specific afferent systems in the cat. Archives of Neurology and Psychiatry, 75, 122-131. Retrieved from http://archneurpsyc.highwire.org/cgi/reprint/75/2/122

Purpura, D. P. (1956b). Electrophysiological analysis of psychotogenic drug action. II. General nature of lysergic acid diethylamide (LSD) action on central synapses. Archives of Neurology and Psychiatry, 75,
132-141. Retrieved from http://archneurpsyc.ama-assn.org/cgi/reprint/ $75 / 2 / 132$

Purves, D., Paydarfar, J. A., \& Andrews, T. J. (1996). The wagon wheel illusion in movies and reality. PNAS: Proceedings of the National Academy Sciences, USA, 93, 3693-3697. doi:10.1073/pnas.93.8.3693

Qubbaj, M. R., \& Jirsa, V. K. (2007). Neural field dynamics with heterogeneous connection topologies. Physical Review Letters, 98, Article 238102. doi:10.1103/PhysRevLett.98.238102

Ray, P. G., Meador, K. J., Epstein, C. M., Loring, D. W., \& Day, L. J. (1998). Magnetic stimulation of visual cortex: Factors influencing the perception of phosphenes. Journal of Clinical Neurophysiology, 15, 351-357. doi:10.1097/00004691-199807000-00007

Reggia, J. A., \& Montgomery, D. (1996). A computational model of visual hallucinations in migraine. Computers in Biology and Medicine, 26 133-141. doi:10.1016/0010-4825(95)00051-8

Remole, A. (1971). Luminance thresholds for subjective patterns in a flickering field: Effects of wavelength. Journal of the Optical Society of America, 61, 1164-1168. doi:10.1364/JOSA.61.001164

Remole, A. (1973). Subjective patterns in a flickering field: Binocular vs monocular observation. Journal of the Optical Society of America, 63, 745-748. doi:10.1364/JOSA.63.000745

Richards, W. (1971, May). The fortification illusions of migraines. Scientific American, 224(5), 88-96. doi:10.1038/scientificamerican0571-88

Richardson, K. A., Schiff, S. J., \& Gluckman, B. J. (2005). Control of traveling waves in the mammalian cortex. Physical Review Letters, 94, Article 028103. doi:10.1103/PhysRevLett.94.028103

Riggs, L. A., Merton, P. A., \& Morton, H. B. (1974). Suppression of visual phosphenes during saccadic eye movements. Vision Research, 14, $997-$ 1011. doi:10.1016/0042-6989(74)90169-2

Ringach, D. L. (2003, October 30). Neuroscience: States of mind. Nature, 425, 912-913. doi:10.1038/425912a

Ringach, D. L. (2009). Spontaneous and driven cortical activity: Implications for computation. Current Opinion in Neurobiology, 19, 439-444. doi:10.1016/j.conb.2009.07.005

Rule, M., Stoffregen, M., \& Ermentrout, B. (2011). A model for the origin and properties of flicker-induced geometric phosphenes. PLoS Computational Biology, 7, Article e1002158. doi:10.1371/journal.pcbi.1002158 Sacks, O. (1995a). Migraine. London, England: Picador.

Sacks, O. (with Seigel, R. M.). (1995b). Migraine aura and hallucinatory constants. In O. Sacks (Author), Migraine (pp. 273-297). London, England: Picador.

Sanderson, A. R., Kirby, R. M., Johnson, C. R., \& Yang, L. (2006) Advanced reaction-diffusion models for texture synthesis. Journal of Graphics, GPU, and Game Tools, 11, 47-71. doi:10.1080/ 2151237X.2006.10129222

Sannita, W. R., Narici, L., \& Picozza, P. (2006). Positive visual phenomena in space: A scientific case and a safety issue in space travel. Vision Research, 46, 2159-2165. doi:10.1016/j.visres.2005.12.002

Schwartz, E. L. (1977). Spatial mapping in the primate sensory projection Analytic structure and relevance to perception. Biological Cybernetics, 25, 181-194. doi:10.1007/BF01885636

Schwartz, E. L. (1980). Computational anatomy and functional architecture of striate cortex: A spatial mapping approach to perceptual coding Vision Research, 20, 645-669. doi:10.1016/0042-6989(80)90090-5

Schwartz, E. L. (1994). Topographical mapping in primate visual cortex: History, anatomy, and computation. In D. H. Kelly (Ed.), Visual science and engineering: Models and applications (pp. 293-359). New York, NY: Marcel Dekker.

Segel, L. A., \& Jackson, J. L. (1972). Dissipative structure: An explanation and an ecological example. Journal of Theoretical Biology, 37, 545-559. doi:10.1016/0022-5193(72)90090-2

Segel, L. A., \& Levin, S. A. (1976). Application of nonlinear stability theory to the study of the effects of diffusion on predator-prey interactions. In R. A. Piccirrelli (Ed.), Topics in statistical mechanics and 
biophysics: A memorial to Julius L. Jackson (pp. 123-152). New York, NY: American Institute of Physics.

Shevelev, L. A., Kamenkovich, V. M., Bark, E. D., Verkhlutov, V. M., Sharaev, G. A., \& Mikhailova, E. S. (2000). Visual illusions and travelling alpha waves produced by flicker at alpha frequency. International Journal of Psychophysiology, 39, 9-20. doi:10.1016/S0167-8760 (00)00105-7

Shoji, H., Iwasa, Y., Mochizuki, A., \& Kondo, S. (2002). Directionality of stripes formed by anisotropic reaction-diffusion models. Journal of Theoretical Biology, 214, 549-561. doi:10.1006/jtbi.2001.2480

Siegel, R. K. (1992). Fire in the brain: Clinical tales of hallucination. New York, NY: Penguin.

Siegel, R. K., \& Jarvik, M. E. (1975). Drug-induced hallucinations in animals and man. In R. K. Siegel, \& L. J. West (Eds.), Hallucinations (pp. 81-162). New York, NY: Wiley.

Smythies, J. R. (1959a). The stroboscopic patterns: I. The dark phase. British Journal of Psychology, 50, 106-116. Retrieved from http:// pao.chadwyck.co.uk/PDF/1298215949727.pdf

Smythies, J. R. (1959b). The stroboscopic patterns: II. The phenomenology of the bright phase and after-images. British Journal of Psychology, 50, 305-324. Retrieved from http://pao.chadwyck.co.uk/PDF/ 1298215661643.pdf

Smythies, J. R. (1960). The stroboscopic patterns: III. Further experiments and discussion. British Journal of Psychology, 51, 247-255. Retrieved from http://pao.chadwyck.co.uk/PDF/1298216224341.pdf

Stwerka, S. A. (1993). The stroboscopic patterns as dissipative structures. Neuroscience and Biobehavioral Reviews, 17, 69-78. doi:10.1016/ S0149-7634(05)80231-3

Swindale, N. V., Matsubara, J. A., \& Cynader, M. S. (1987). Surface organization of orientation and direction selectivity in cat area 18 . Journal of Neuroscience, 7, 1414-1427. Retrieved from http:// www.jneurosci.org/cgi/reprint/7/5/1414

Tass, P. (1995). Cortical pattern formation during visual hallucinations. Journal of Biological Physics, 21, 177-210. doi:10.1007/BF00712345

Tass, P. (1997). Oscillatory cortical activity during hallucination. Journal of Biological Physics, 23, 21-66. doi:10.1023/A:1004990707739

Thom, R. (1975). Structural stability and morphogenesis. Reading, MA: W. A. Benjamin.

Thurlow, H. J., \& Girvin, J. P. (1971). Use of anti-epileptic medication in treating "flashbacks" from hallucinogenic drugs. Canadian Medical Association Journal, 105, 947-948. Retrieved from http://www.ncbi.nlm .nih.gov/pmc/articles/PMC1931731

Tootell, R. B. H., Hadjikhani, N. K., Vanduffel, W., Liu, A. K., Mendola, J. D., Sereno, M. I., \& Dale, A. M. (1998). Functional analysis of primary visual cortex (V1) in humans. PNAS: Proceedings of the National Academy of Sciences, USA, 95, 811-817. doi:10.1073/pnas 95.3.811

Tootell, R. B. H., Silverman, M. S., Switkes, E., \& DeValois, R. L. (1982, November 26). Deoxyglucose analysis of retinotopic organization in primate striate cortex. Science, 218, 902-904. Retrieved from http:// www.jstor.org/stable/1689042

Tootell, R. B. H., Switkes, E., Silverman, M. S., \& Hamilton, S. L. (1988). Functional anatomy of macaque striate cortex: II. Retinotopic organization. Journal of Neuroscience, 8, 1531-1568. Retrieved from http:// jneurosci.org/content/8/5/1531.long

Treisman, A. (1996). The binding problem. Current Opinion in Neurobiology, 6, 171-178. doi:10.1016/S0959-4388(96)80070-5

Turing, A. M. (1952). The chemical basis of morphogenesis. Philosophical Transactions of the Royal Society of London: Series B. Biological Sciences, 237, 37-72. doi:10.1098/rstb.1952.0012

Tyler, C. W. (1978). Some new entopic phenomena. Vision Research, 18, 1633-1639. doi:10.1016/0042-6989(78)90255-9

Tyler, C. W., \& Hardage, L. (1998). Long-range twinkle induction: An achromatic rebound effect in the magnocellular processing system? Perception, 27, 203-214. doi: $10.1068 / \mathrm{p} 270203$

Tynan, P., \& Sekular, R. (1975, May 30). Moving visual phantoms: A new contour completion effect. Science, 188, 951-952. doi:10.1126/ science. 1138365

van Harreveld, A., \& Stamm, J. S. (1955). Cortical responses to metrazol and sensory stimulation in the rabbit. Electroencephalography and Clinical Neurophysiology, 7, 363-370. doi:10.1016/0013-4694(55)90005-5

Vaphiades, M., Celesia, G. G., \& Brigell, M. G. (1996). Positive spontaneous visual phenomena limited to hemianopic fields in lesions of central visual pathways. Neurology, 47, 408-417. Retrieved from http:// neurology.org/content/47/2/408.full.pdf + html

Vernon, J. A. (1963). Inside the black room. New York, NY: Clarkson Potter.

Vidyasagar, T. R., Buzás, P., Kisvárday, Z. F., \& Eysel, U. T. (1999, June 3). Release from inhibition reveals the visual past. Nature, 399, 422 doi: $10.1038 / 20836$

Volkmann, F. C. (1986). Human visual suppression. Vision Research, 26 , 1401-1416. doi:10.1016/0042-6989(86)90164-1

Wackermann, J., Pütz, P., \& Allefeld, C. (2008). Ganzfeld-induced hallucinatory experience, its phenomenology and cerebral electrophysiology. Cortex, 44, 1364-1378. doi:10.1016/j.cortex.2007.05.003

Wade, N. J., \& Brozek, J. (2001). Purkinje's vision: The dawning of neuroscience. Mahwah, NJ: Erlbaum.

Walter, V. J., \& Walter, W. G. (1949). The central effects of rhythmic sensory stimulation. Electroencephalography and Clinical Neurophysiology, 1, 57-86. doi:10.1016/0013-4694(49)90164-9

Walter, W. G. (1956, April 14). Colour illusions and aberrations during stimulation by flickering light. Nature, 177, 710. doi:10.1038/177710a0

Wede, J., \& Francis, G. (2006). The time course of visual afterimages: Data and theory. Perception, 35, 1155-1170. doi:10.1068/p5521

Werner, G. (2007). Metastability, criticality and phase transitions in brain and its models. Biosystems, 90, 496-508. doi:10.1016/j.biosystems.2006.12.001

Wiener, M. C. (1994). Hallucinations, symmetry, and the structure of primary visual cortex: A bifurcation theory approach (Doctoral dissertation). University of Chicago, Chicago, IL.

Wilkinson, F. (2004). Auras and other hallucinations: Windows on the visual brain. Progress in Brain Research, 144, 305-320. doi:10.1016/ S0079-6123(03)14402-1

Wilkinson, F., Feindel, A., \& Grivell, J. E. (1999). Mapping of visual migraine auras into cortical coordinates [Abstract]. Headache, 39, 386.

Wilkinson, F., Karanovic, O., \& Wilson, H. R. (2008). Binocular rivalry in migraine. Cephalalgia, 28, 1327-1338. doi:10.1111/j.14682982.2008.01696.x

Williamson, P. D., Thadani, V. M., Darcey, T. M., Spenser, D. D., \& Mattson, R. H. (1992). Occipital lobe epilepsy: Clinical characteristics, seizure spread patterns, and results of surgery. Annals of Neurology, 31, 3-13. doi:10.1002/ana.410310103

Wilson, H. R. (1999). Spikes, decisions and actions: Dynamical foundations of neuroscience. Oxford, England: Oxford University Press.

Wilson, H. R., \& Cowan, J. D. (1972). Excitatory and inhibitory interactions in localized populations of model neurons. Biophysical Journal, 12, 1-24. doi:10.1016/S0006-3495(72)86068-5

Wilson, H. R., \& Cowan, J. D. (1973). A mathematical theory of the functional dynamics of cortical and thalamic nervous tissue. Kybernetik, 13, 55-80. doi:10.1007/BF00288786

Winters, W. D. (1975). The continuum of CNS excitatory states and hallucinosis. In R. K. Siegel \& L. J. West (Eds.), Hallucinations. Behavior, experience and theory (pp. 53-70). New York, NY: Wiley.

Wolff, J. G., Delacour, J., Carpenter, R. H. S., \& Brindley, G. S. (1968). The patterns seen when alternating electric current is passed through the eye. Quarterly Journal of Experimental Psychology, 20, 1-10. doi: $10.1080 / 14640746808400122$

Wolters, E. C., \& Berendse, H. W. (2001). Management of psychosis in 
Parkinson's disease. Current Opinion in Neurology, 14, 499-504. doi: 10.1097/00019052-200208000-00011

Young, R. S. L., Cole, R. E., Gamble, M., \& Rayner, D. M. (1975). Subjective patterns elicited by light flicker. Vision Research, 15, 12911293. Retrieved from http://ncbi.nlm.nih.gov/pubmed/1198944
Zubek, J. P. (1969). Sensory deprivation: Fifteen years of research. New York, NY: Appleton-Century-Crofts.

Zuckerman, M., \& Cohen, N. (1964). Sources of reports of visual and auditory sensations in perceptual-isolation experiments. Psychological Bulletin, 62, 1-20. doi:10.1037/h0048599

\section{Appendix}

\section{The Nonlinear Transform From Retinal to Cortical Coordinates}

Geometry is a magic that works.—René Thom (1975)

It has long been known that resolution of stimuli grows progressively worse with the distance on retina away from the fovea (fixation point). Several factors contribute to this: Receptive field centers in foveal retina are smaller than in the periphery, foveal optics are better than peripheral optics, retinal ganglion cell density is higher near fovea, and visual cortex devotes more neurons to foveal inputs to peripheral ones. These factors fit neatly together; the cortex devotes its resources in accordance to the availability of retinal information. Thus, the cortex magnifies the foveal representation and progressively minifies the peripheral representation. Originally, cortical magnification $C_{M}$ was thought of as a ratio of neural image size on cortex to optical image size on retina. An application of this is $M$-scaling: the physical magnification that must be applied to a stimulus at a peripheral location in the retina for a particular perceptual task to be completed that could be completed with an unmagnified stimulus presented to the fovea. For example, contrast sensitivity is relatively constant if spatial frequency is expressed in cycles/millimeter on visual cortex. The cortical magnification factor can also be inferred from the scaling of fortification serrations in migraine (Grüsser, 1995).

In general, cortical magnification $C_{M}$ at a distance $r$ (in degrees of visual angle) from the fovea along a constant retinal direction $\theta$, is fit by a simple monotonically decreasing function

$$
C_{M}=k /(r+a)
$$

Because $C_{M}$ can be defined as a ratio of small changes of cortical and retinal extent, it resembles a differential. If we naively integrate a function of this form - as Fechner did for Weber's law (see Billock \& Tsou, 2011, on Fechner's law)—a logarithmic function is obtained. However, since both the retina and cortex are twodimensional sheets and isotropy is not guaranteed, the situation is a little more complex (Schwartz, 1994). In 1977, two independent investigators noted that the cortical magnification factor can be integrated in such a way as to yield a sensible mapping from the polar $(r, \theta)$ retina to the more rectilinear $(x, y)$ cortex (Cowan, 1977; Schwartz, 1977).

$$
x=\beta \ln \left[\left(\sqrt{ }(\varepsilon r)+\left(w_{o}^{2}+\varepsilon r^{2}\right)^{1 / 2}\right) / 2 w_{o}\right] ; y=\beta r \theta /\left(w_{o}^{2}+\varepsilon r^{2}\right)^{1 / 2},
$$

where $r$ is radial distance (in degrees of visual angle) on retina from fovea, $\theta$ is the angular direction from the fovea, $w_{o}$ is the mean diameter of foveal receptive fields, $\varepsilon$ is the rate of increase of that diameter with $r, d$ is the density of receptive field overlap, and $\beta=(4 d / \pi \varepsilon)^{1 / 2}$. Equation $\mathrm{A} 2$ is the version that was employed by Ermentrout and Cowan (1979) and Ermentrout (1984); variations can be found in other studies, but for our purposes, the differences are of no consequence. For distances greater than one degree of visual angle from the fovea (e.g., for all but a tiny portion of the ca. $135^{\circ}$ vertical $\times 150^{\circ}$ horizontal degree visual field), this function is well approximated as $x=\beta \ln \left(r \sqrt{ } \varepsilon / w_{o}\right) ; y=\beta \theta$. This mapping is sometimes called a complex logarithmic function (because the same form can be used to represent the logarithm of a complex number like $\ln [r, i \theta]$; Cowan, 1977; Schwartz, 1977). This conceptual advance did not get the attention it deserved until Tootell, Silverman, Switkes, and DeValois (1982) found a direct and astonishingly convincing way to image the mapping in primates. They created a polar grid visual stimulus consisting of radial lines intersecting logarithmically spaced concentric circles. Markings on the grid continually reversed in contrast, assuring that they would activate both on- and off-cells and both sustained- and transient-cells for maximum neural response. The stimuli were shown for $30 \mathrm{~min}$ to one eye of a macaque who had been dosed with radioactive ${ }^{14} \mathrm{C}$-labeled 2-deoxy-D-glucose. Increased neural activity led to increased uptake of the radioactive glucose; after metabolism, the radioactive tag accumulated in the active neurons. The animal was then sacrificed, and its occipital cortex was extracted and pressed against a photographic plate, which was exposed by emitted radiation (see Figure A1). The delineation between stimulated and inactive cortex is remarkably crisp. Although modern imaging techniques allow the same mapping to be studied in intact humans (see Figure 5), this remains the most convincing single direct demonstration of any neural inference that we are aware of. ${ }^{\mathrm{A} 1}$

Although the nonlinear retinocortical mapping has important implications for how the cortex may be organized to process spatial information (Schwartz, 1977, 1980), for our purposes its most important implication is that three very different kinds of

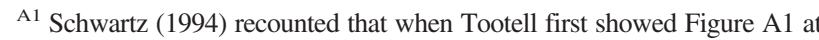
a conference (the Association for Research in Vision and Ophthalmology annual meeting, ca. 1980), the audience spontaneously stood and applauded.
} 

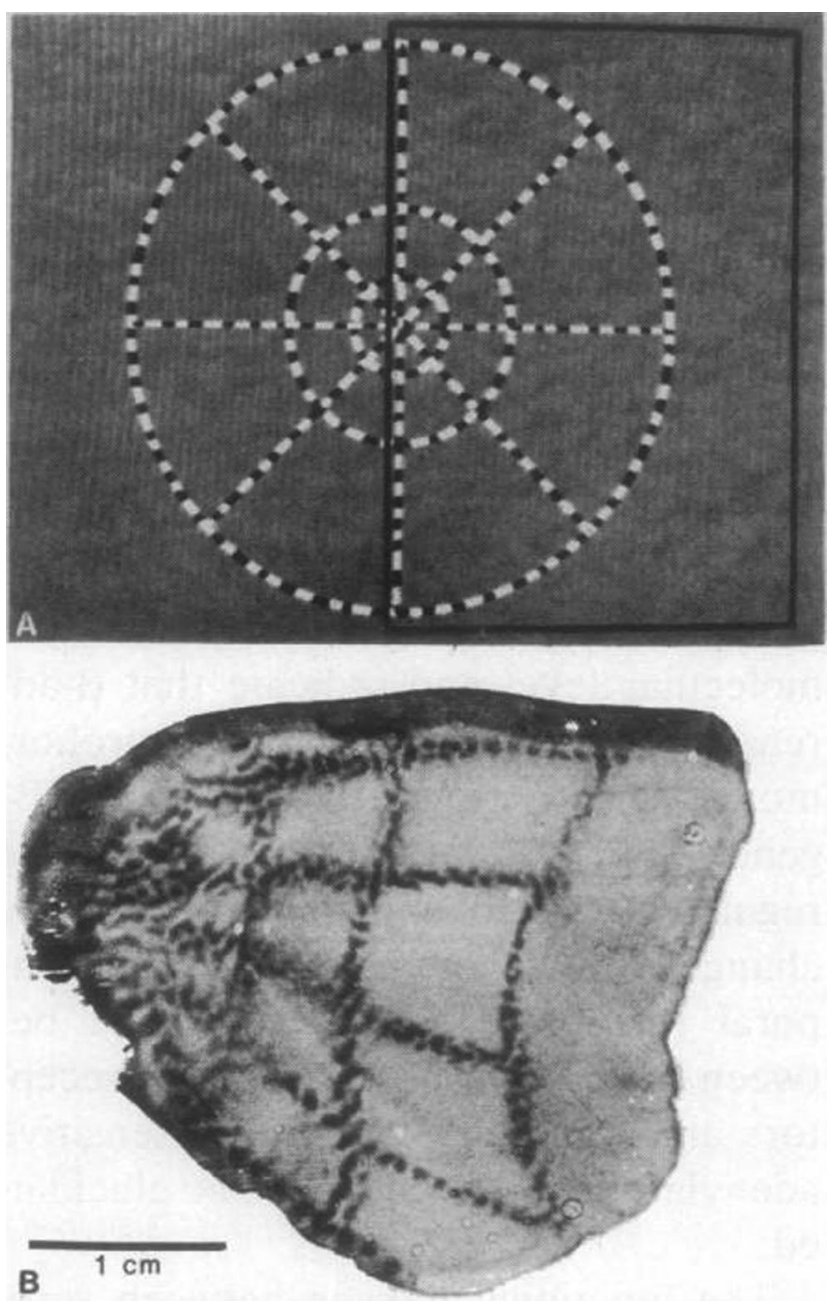

Figure A1. The clearest and most compelling demonstration of the nonlinear mapping from retinal to cortical coordinates. Equation A2 is a complex logarithmic mapping from retinal polar coordinates to cortical Cartesian coordinates, which formed the basis for Ermentrout and Cowan's (1979) hypothesis. The polar web pattern on the top (A) was used to stimulate the visual system of a macaque treated with radioactively labeled glucose. After sufficient time to accumulate the tracer in the most active cells (for details, see the Appendix), the radiograph (B) of the animal's cortex was made. The radial and concentric features of the polar web stimulation on retina are transformed to the nearly rectilinear grid shown on V1. Almost two decades later, it became possible for Tootell et al. (1998) to recreate this demonstration noninvasively in living humans using functional magnetic resonance imaging (see Figure 5), but the clarity of this early demonstration has not yet been equaled. From "Deoxyglucose Analysis of Retinotopic Organization in Primate Striate Cortex," by R. B. H. Tootell, M. S. Silverman, E. Switkes, \& R. L. DeValois, 1982, Science, 218, p. 902 . Copyright 1982 by the American Association for the Advancement of Science. Reprinted with permission from AAAS. 
retinal stimulus patterns would result in identical cortical activity patterns: fan shapes, concentric circles, and spirals imaged on retina all map to cortex as parallel stripes of cortical activity. Some corollaries of this are the following: (a) If parallel stripes of cortical activity form spontaneously, their percept is governed by orientation of the stripes on cortex (Ermentrout \& Cowan, 1979). (b) Perceptually opponent shapes, like fan shapes and concentric circles, are associated with orthogonal patterns of cortical activity (as are clockwise and counterclockwise spirals). (c) Scrolling stripe patterns on cortex leads to rotating fan shapes and spirals and to expanding/contracting concentric circles. (d) Rotating stripes of cortical activity morphs per- cepts-fan shapes twist into spirals, which progressively tighten until becoming concentric circles; multistability between hallucinatory patterns could result. Much hallucinatory diversity can thus be explained by the interaction of cortical stripe formation with the nonlinear retinocortical mapping.
Received May 13, 2011

Revision received October 12, 2011

Accepted November 16, 2011

\section{ORDER FORM}

Start my 2012 subscription to Psychological Bulletin ISSN: 0033-2909

\section{$\$ 109.00$ APA MEMBER/AFFILIATE \\ $\$ 294.00$ INDIVIDUAL NONMEMBER \\ $\$ 837.00$ INSTITUTION}

In DC and $M D$ add $6 \%$ sales tax

TOTAL AMOUNT DUE

Subscription orders must be prepaid. Subscriptions are on a calendar year basis only. Allow 4-6 weeks for delivery of the first issue. Call for international subscription rates.

SEND THIS ORDER FORM TO

American Psychological Association

Subscriptions

750 First Street, NE

Washington, DC 20002-4242

AMERICAN PSYCHOLOGICAL ASSOCIATION
Call 800-374-2721 or 202-336-5600

Fax 202-336-5568 :TDD/TTY 202-336-6123

For subscription information,

e-mail: subscriptions@apa.org $\square$ Check enclosed (make payable to APA)

Charge my: $\square$ Visa $\square$ MasterCard $\square$ American Express

Cardholder Name

Card No. _ Exp. Date

Signature (Required for Charge)

\section{Billing Address}

Street

City State Zip

Daytime Phone

E-mail

\section{Mail To}

Name

Address

City _ State _ Zip _ _

APA Member \# 